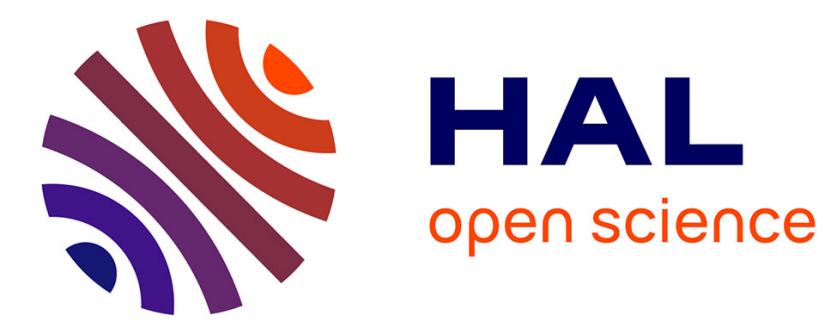

\title{
Separation by Convex Pseudo-Circles
}

N Chevallier, A Fruchard, D Schmitt, J.-C Spehner

\section{To cite this version:}

N Chevallier, A Fruchard, D Schmitt, J.-C Spehner. Separation by Convex Pseudo-Circles. 2015. hal-01108235

\section{HAL Id: hal-01108235 \\ https://hal.science/hal-01108235}

Preprint submitted on 22 Jan 2015

HAL is a multi-disciplinary open access archive for the deposit and dissemination of scientific research documents, whether they are published or not. The documents may come from teaching and research institutions in France or abroad, or from public or private research centers.
L'archive ouverte pluridisciplinaire HAL, est destinée au dépôt et à la diffusion de documents scientifiques de niveau recherche, publiés ou non, émanant des établissements d'enseignement et de recherche français ou étrangers, des laboratoires publics ou privés. 


\title{
Separation by Convex Pseudo-Circles
}

\author{
N. Chevallier, A. Fruchard, D. Schmitt, and J.-C. Spehner \\ Laboratoire LMIA, Université de Haute-Alsace \\ 4, rue des Frères Lumière, 68093 Mulhouse Cedex, France \\ Nicolas.Chevallier@uha.fr, Augustin.Fruchard@uha.fr, \\ Dominique.Schmitt@uha.fr, Jean-Claude.Spehner@uha.fr
}

\begin{abstract}
Let $S$ be a finite set of $n$ points in the plane in general position. We prove that every inclusion-maximal family of subsets of $S$ separable by convex pseudo-circles has the same cardinal $\left(\begin{array}{l}n \\ 0\end{array}\right)+\left(\begin{array}{l}n \\ 1\end{array}\right)+\left(\begin{array}{l}n \\ 2\end{array}\right)+\left(\begin{array}{l}n \\ 3\end{array}\right)$. This number does not depend on the configuration of $S$ and is the same as the number of subsets of $S$ separable by true circles. Buzaglo, Holzman, and Pinchasi showed that it is an upper bound for the number of subsets separable by (non necessarily convex) pseudo-circles.

Actually, we first count the number of elements in a maximal family of $k$-subsets of $S$ separable by convex pseudo-circles, for a given $k$. We show that Lee's result on the number of $k$-subsets separable by true circles still holds for convex pseudo-circles. In particular, this means that the number of $k$-subsets of $S$ separable by a maximal family of convex pseudo-circles is an invariant of $S$ : It does not depend on the choice of the maximal family.

To prove this result, we introduce a graph that generalizes the dual graph of the order- $k$ Voronoi diagram, and whose vertices are the $k$-subsets of $S$ separable by a maximal family of convex pseudo-circles. In order to count the number of vertices of this graph, we first show that it admits a planar realization which is a triangulation. It turns out (but is not detailed in the present paper) that these triangulations are the centroid triangulations Liu and Snoeyink conjectured to construct.
\end{abstract}

\section{Introduction}

Given a set $S$ of $n$ points in the plane in general position, a classical problem in combinatorial and computational geometry consists in searching and counting subsets of $S$ that can be separated from the remaining points by different types of lines.

The case of straight lines has been extensively studied. For a fixed $k \in\{0, \ldots, n\}$, the exact number $a_{k}(S)$ of $k$-sets, i.e. subsets of $k$ points separable by straight lines, is not completely understood. Nowadays, the best upper bound for the maximum of $a_{k}(S)$ over all configurations of the $n$ points is $O\left(n k^{1 / 3}\right)$, due to Dey [6]. In [18], Tóth constructs examples with $n 2^{\Omega(\sqrt{\log k})}$ $k$-sets $(k \geq 1)$. It is noticeable that the sum over all $k$ of the numbers of $k$-sets of $S$, i.e. the total number of subsets of $S$ separable by straight lines, does not depend on the position of the points but only on $n$ : We have $\sum_{k=0}^{n} a_{k}(S)=n(n-1)+2$.

It seems to be known by specialists, although nowhere explicitly stated up to our knowledge, that the total number of subsets separable by circles does not depend on the position of the points, too. This number turns out to be the cake number $c(3, n)=\left(\begin{array}{l}n \\ 0\end{array}\right)+\left(\begin{array}{l}n \\ 1\end{array}\right)+\left(\begin{array}{l}n \\ 2\end{array}\right)+\left(\begin{array}{l}n \\ 3\end{array}\right)$, i.e. the maximal number of cells in an arrangement of $n$ planes in 3-dimensional space [17], see Section 6.

A natural generalization is to count the number of subsets of $S$ separable by pseudo-circles. A family $\mathcal{F}$ of subsets of $S$ is said to be separable by pseudo-circles if there exists a family $\mathcal{C}$ of 
Jordan curves, pairwise intersecting at most twice, such that each element of $\mathcal{F}$ is separable by an element of $\mathcal{C}$. Intuitively, one could believe that families of pseudo-circles provide a greater number of separable subsets than circles. In fact, using the theory of VC-dimension of Vapnik and Chervonenkis $[19,14,16]$, Buzaglo, Holzman, and Pinchasi prove in [4] that $c(3, n)$ is an upper bound for pseudo-circles, see Section 6.1 at the end of the present article.

Surprisingly, it is far from evident whether $c(3, n)$ is also a lower bound for any inclusionmaximal family of subsets of $S$ separable by pseudo-circles. Up to our knowledge, the question remains open. In this article, we prove this for convex pseudo-circles. We refer to Section 6.4 for a discussion when the word "convex" is dropped.

Theorem 1.1. Let $S$ be a set of $n$ points in the plane in general position. Every inclusionmaximal family $\mathcal{F}$ of subsets of $S$ separable by convex pseudo-circles has $c(3, n)=\left(\begin{array}{l}n \\ 0\end{array}\right)+\left(\begin{array}{l}n \\ 1\end{array}\right)+$ $\left(\begin{array}{l}n \\ 2\end{array}\right)+\left(\begin{array}{l}n \\ 3\end{array}\right)$ elements.

In fact, we first consider families of $k$-subsets separable by convex pseudo-circles. For each fixed $k \in\{1, \ldots, n-1\}$, Lee proved in [9] that any $n$-point set $S$ of the plane in general position admits $2 k n-n-k^{2}+1-\sum_{i=1}^{k-1} a_{i}(S) k$-subsets separable by true circles (with the convention $\sum_{1}^{0}=0$ ). We prove that this number also holds for all maximal families of $k$-subsets separable by convex pseudo-circles.

Theorem 1.2. Let $S$ be a set of $n$ points in the plane in general position and $k \in\{1, \ldots, n\}$. Every inclusion-maximal family of $k$-subsets of $S$ separable by convex pseudo-circles admits $2 k n-n-k^{2}+1-\sum_{i=1}^{k-1} a_{i}(S)$ elements.

To prove these results, we first characterize convex pseudo-circle separability in terms of convex hulls (Section 2). We show that a family $\mathcal{F}$ of subsets of $S$ is separable by convex pseudo-circles if $\operatorname{conv}(T) \cap S=T$ for all $T \in \mathcal{F}$, and $\operatorname{conv}\left(T \backslash T^{\prime}\right) \cap \operatorname{conv}\left(T^{\prime} \backslash T\right)=\emptyset$ for all $T, T^{\prime} \in \mathcal{F}$. We also characterize in a similar way families of pairs $(P, Q)$ of subsets of $S$ determined by convex pseudo-circles passing through the points of $Q$, containing $P$, and excluding $S \backslash(P \cup Q)$. Such pairs are called convex pairs.

We then introduce a graph that generalizes the dual of the order- $k$ Voronoi diagram (Sections 3 and 4). The vertices of this graph are the elements of size $k$ in a family of subsets of $S$ separable by a maximal family $\mathcal{C}$ of convex pseudo-circles. The edges of the graph are the convex pairs of the form $(P,\{s, t\})$, with $|P|=k-1$, determined by convex pseudo-circles compatible with $\mathcal{C}$. The edge $(P,\{s, t\})$ connects the two vertices $P \cup\{s\}$ and $P \cup\{t\}$. The key result of the article, Theorem 3.9, is that this graph admits a planar geometric realization which induces a triangulation. Proposition 3.4 already shows that the edges of this realization are disjoint. The main difficulty is to show that every edge is incident to a triangle, see Theorem 3.7. The long and tricky proof of this result can be found in Section 5 .

Two important contributions of our work are, firstly, the notion of convex pair and, secondly, the characterization of the separability by convex pseudo-circles in terms of convex hulls. The systematic use of convex hulls has the advantage to be generalizable straightforwardly in higher dimension, without using the interesting but subtle notion of pseudo-sphere.

Definition 1.3. Given a finite subset $S$ of $\mathbb{R}^{d}$, a family $\mathcal{F}$ of subsets of $S$ is said to be separable by convex pseudo-spheres if $\operatorname{conv}(T) \cap S=T$ for all $T \in \mathcal{F}$ and $\operatorname{conv}\left(T \backslash T^{\prime}\right) \cap \operatorname{conv}\left(T^{\prime} \backslash T\right)=\emptyset$ for all $T, T^{\prime} \in \mathcal{F}$.

A natural example is the family of all subsets of $S$ separable by usual $(d-1)$-dimensional spheres, see Section 6. Our main result about separation by pseudo-spheres and spheres is the following, proven in Section 6.

Theorem 1.4. Let $d \geq 2$ and let $S$ be a set of $n$ points in $\mathbb{R}^{d}$ in general position, i.e. no $d+1$ of them in the same hyperplane. Then every family $\mathcal{F}$ of subsets of $S$ separable by convex 
pseudo-spheres has at most $c(d+1, n)=\left(\begin{array}{l}n \\ 0\end{array}\right)+\left(\begin{array}{c}n \\ 1\end{array}\right)+\cdots+\left(\begin{array}{c}n \\ d+1\end{array}\right)$ elements. If moreover no $d+2$ points of $S$ lie on a same sphere, then the bound is attained for the family of all subsets of $S$ separable by $(d-1)$-dimensional spheres.

Let us mention some related works in the literature. In [4], Buzaglo, Holzman, and Pinchasi consider a family of subsets of $S$ in the plane separable by Jordan curves pairwisely intersecting properly, at most $s$ times, and such that pairwise intersections of the Jordan disks are empty or connected. They prove that such a family has VC-dimension at most $s+1$, and hence contains at most $\sum_{i=0}^{s+1}\left(\begin{array}{c}n \\ i\end{array}\right)$ elements. Our result is that this upper bound is attained for any maximal family, in the case of convex curves and for $s=2$. It would be interesting to know to what extent this remains valid in general. The authors of [4] also consider $k$-subsets separable by pseudo-circles that all pairwise intersect and they show that their number is $O(k n)$.

In [12], Pinchasi and Rote consider a slightly different and more restrictive notion of convex pseudo-circles: A family $\mathcal{F}$ of subsets of $S$ is separable by convex pseudo-circles in their sense, if every set $T$ in $\mathcal{F}$ is the intersection of $S$ with a convex set, and if both $\operatorname{conv}(T) \backslash \operatorname{conv}\left(T^{\prime}\right)$ and $\operatorname{conv}\left(T^{\prime}\right) \backslash \operatorname{conv}(T)$ are empty or connected for all $T, T^{\prime} \in \mathcal{F}$. They prove that, if no member of $\mathcal{F}$ is contained in another, then $\mathcal{F}$ consists of at most $4\left(\begin{array}{l}n \\ 2\end{array}\right)+1$ elements. They also present an example with $\left(\begin{array}{c}n+1 \\ 2\end{array}\right)$ elements.

In [9], Lee provides an algorithm to construct the order- $k$ Voronoi diagram. This algorithm can be dualized for order- $k$ centroid Delaunay triangulations [15]. Liu and Snoeyink propose in [10] to generalize this dual algorithm, giving rise to other order- $k$ centroid triangulations when their algorithm does not fail. They conjecture that their algorithm actually never fails and they prove this for $k \leq 3$. A proof for all $k$, but for particular centroid triangulations, is given in [8]. It turns out that the triangulations defined in the present article are precisely the ones constructed by the algorithm of Liu and Snoeyink. As a consequence, this article serves as the basis for the proof that their algorithm never fails. This will be done in an article in preparation.

An extended abstract of the present article has been presented in [5].

\section{Characterization of convex pseudo-circle separability}

For a subset $E$ of the plane $\mathbb{R}^{2}, \operatorname{conv}(E)$ denotes its convex hull, $\partial E$ its boundary, $\operatorname{relint}(E)$ its relative interior, and $\bar{E}$ its closure.

If $a$ and $b$ are two distinct points in the plane, $(a b)$ is the straight line passing through $a, b$ oriented from $a$ to $b$, and $[a, b]$ (resp. $] a, b[$ ) is the closed (resp. open) segment connecting $a$ and $b$.

Given an oriented straight line $\Delta$ in the plane, $\Delta^{+}$(resp. $\Delta^{-}$) denotes the open half plane on the left (resp. right) of $\Delta$.

For a Jordan curve $\gamma$ of $\mathbb{R}^{2}$, disk $(\gamma)$ denotes the bounded open component of $\mathbb{R}^{2} \backslash \gamma$.

In the whole article, $S$ denotes a finite set of $n$ points of the plane, no three of them being collinear.

\subsection{Convex subsets}

The aim of this subsection is to show that subsets of $S$ separable by convex pseudo-circles can be characterized using convex hulls only. This will avoid manipulating curves in the remainder of the article.

Definition 2.1. We say that a subset $T$ of $S$ is separable by a convex pseudo-circle if there exists a convex Jordan curve $\gamma$ that avoids $S$ such that $S \cap \operatorname{disk}(\gamma)=T$.

Such a subset $T$ is also called convex in $S$ and $\gamma$ is called a separating curve of $T$.

Proposition 2.2. A subset $T$ is convex in $S$ if and only if $\operatorname{conv}(T) \cap S=T$. 
Proof. By definition, if $T$ is a convex subset of $S$ with a separating curve $\gamma$, then we have

$$
T=T \cap S \subseteq \operatorname{conv}(T) \cap S \subseteq \operatorname{conv}(\operatorname{disk}(\gamma)) \cap S=\operatorname{disk}(\gamma) \cap S=T .
$$

Conversely, suppose $\operatorname{conv}(T) \cap S=T$. If $|T| \leq 2$, one can include $T$ in a sufficiently small rectangle so that it contains no other point of $S$. The boundary of the rectangle is a separating curve of $T$. If $|T| \geq 3$, one can apply to $T$ a homothety $\varphi$ of center a point of relint $(\operatorname{conv}(T))$ and of ratio $a>1$. For $a$ sufficiently close to 1 , one has $\operatorname{conv}(\varphi(T)) \cap S=T$ and $\partial \operatorname{conv}(\varphi(T)) \cap S=\emptyset$. The polygonal curve $\partial \operatorname{conv}(\varphi(T))$ is a separating curve of $T$.

Definition 2.3. Two convex Jordan curves are said to be compatible if they either intersect properly at exactly two points, meet at exactly one point, or do not meet at all.

Two convex subsets of $S$ that admit two compatible separating curves are said to be compatible.

We say that a family $\mathcal{F}$ of subsets of $S$ is separable by convex pseudo-circles if there exists a set $\mathcal{C}$ of pairwise compatible convex Jordan curves such that each element of $\mathcal{F}$ admits a separating curve in $\mathcal{C}$.

It is worth noting here that the concept of family of subsets separable by convex pseudocircles is a "global" concept. Indeed, in order to determine whether a family $\mathcal{F}$ is separable by convex pseudo-circles, one needs to find a separating curve for each element in $\mathcal{F}$, and all these curves must be compatible with each others. The next proposition is interesting because it shows that it suffices in fact to consider all pairs of elements in $\mathcal{F}$ independently and to find two compatible separating curves for each such pair. The proposition also shows that compatibility can be expressed in terms of intersection of convex hulls; Figure 1 shows some arguments of its proof.

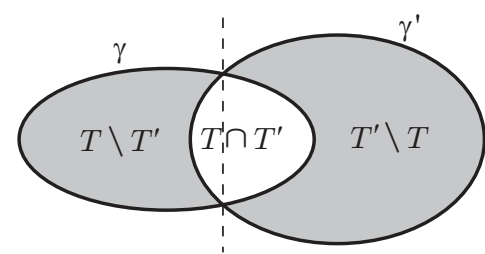

(a)

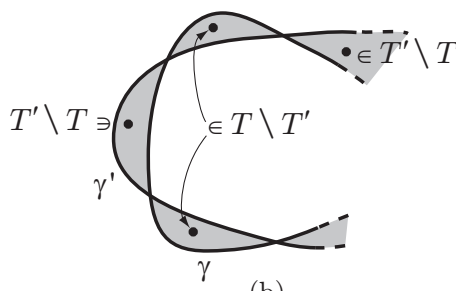

(b)

Figure 1: (a) If $T$ and $T^{\prime}$ are separable by two properly intersecting convex pseudo-circles $\gamma$ and $\gamma^{\prime}$, then $T \backslash T^{\prime}$ and $T^{\prime} \backslash T$ are on both sides of the straight line containing $\gamma \cap \gamma^{\prime}$, and their convex hulls do not meet. (b) Conversely, if two separating convex pseudo-circles $\gamma$ and $\gamma^{\prime}$ of $T$ and $T^{\prime}$ minimize intersections and properly intersect in more than two points, then $\operatorname{disk}(\gamma) \backslash \overline{\operatorname{disk}\left(\gamma^{\prime}\right)}\left(\right.$ resp. $\left.\operatorname{disk}\left(\gamma^{\prime}\right) \backslash \overline{\operatorname{disk}(\gamma)}\right)$ admits at least two connected components meeting $S$, and $\operatorname{conv}\left(T \backslash T^{\prime}\right) \cap \operatorname{conv}\left(T^{\prime} \backslash T\right) \neq \emptyset$.

Proposition 2.4. (i) Two convex subsets $T$ and $T^{\prime}$ of $S$ are compatible if and only if

$$
\operatorname{conv}\left(T \backslash T^{\prime}\right) \cap \operatorname{conv}\left(T^{\prime} \backslash T\right)=\emptyset .
$$

(ii) A family $\mathcal{F}$ of subsets of $S$ is separable by convex pseudo-circles if and only if the elements of $\mathcal{F}$ are pairwise compatible convex subsets of $S$.

Proof. The proof uses arguments independent of the rest of the article and can be skipped at first reading. We divide it in three steps. It is first shown, in (a), that every family of subsets of $S$ separable by convex Jordan curves admits a set of separating curves such that, if $\gamma$ and $\gamma^{\prime}$ are two intersecting curves in the set, then every connected component of $\operatorname{disk}(\gamma) \backslash \overline{\operatorname{disk}\left(\gamma^{\prime}\right)}$ 


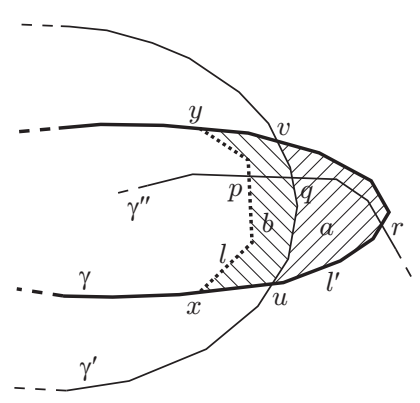

Figure 2: Proof (a) of Proposition 2.4.

meets $S$. Such a component is called an ear in [4]. The results (i) and (ii) are then deduced from (a) in (b) and (c).

(a) Consider a family $\mathcal{F}$ of convex subsets of $S$, compatible or not. From the proof of Proposition 2.2, the elements of $\mathcal{F}$ admit polygonal convex separating curves. One can always perturb the vertices of these curves in such a way that their vertices are distinct and are not in $S$, no three of them are collinear, and no three curves intersect at a same point. Thus, any two of these curves either are disjoint or intersect properly in a finite set of points. Let $\mathcal{C}$ be such a set of separating curves, that minimizes the total number of intersections between its curves. Let $A$ be the set of connected components $a$ of $\operatorname{disk}(\gamma) \backslash \overline{\operatorname{disk}\left(\gamma^{\prime}\right)}$ such that $a \cap S=\emptyset$, for all ordered pairs $\left(\gamma, \gamma^{\prime}\right)$ of intersecting curves of $\mathcal{C}$. We show by contradiction that $A$ is empty.

If $A$ is nonempty, $A$ contains a component $a$ with minimal area. Let $\gamma$ and $\gamma^{\prime}$ be the two curves in $\mathcal{C}$ such that $a$ is a connected component of $\operatorname{disk}(\gamma) \backslash \overline{\operatorname{disk}\left(\gamma^{\prime}\right)}$ and let $T=\operatorname{disk}(\gamma) \cap S$. Let $u$ and $v$ be the two points of $\bar{a} \cap \gamma \cap \gamma^{\prime}$ such that $a \subset(u v)^{-}$, see Figure 2. There exists on $\gamma \cap \operatorname{disk}\left(\gamma^{\prime}\right)$ a point $x$ close to $u$ and a point $y$ close to $v$ such that $\bar{a} \subset(x y)^{-}$, and that $\gamma \cap(x u)^{-}$ and $\gamma \cap(v y)^{-}$are not cut by any curve of $\mathcal{C} \backslash\{\gamma\}$. For all curves of $\mathcal{C} \backslash\left\{\gamma, \gamma^{\prime}\right\}$, let $Z$ be the subset of their vertices included in $\operatorname{disk}(\gamma) \cap \operatorname{disk}\left(\gamma^{\prime}\right) \cap(x y)^{-}$. There exists a convex polygonal curve $l$ from $x$ to $y$ in $\left(\operatorname{disk}(\gamma) \cap \operatorname{disk}\left(\gamma^{\prime}\right) \cap(x y)^{-}\right) \backslash \operatorname{conv}(Z \cup T \cup\{x, y\})$ that intersects the curves of $\mathcal{C}$ properly. Setting $l^{\prime}=\gamma \cap(x y)^{-}, \gamma_{l}=\left(\gamma \backslash l^{\prime}\right) \cup l$ is a convex polygonal curve that intersects the curves of $\mathcal{C} \backslash\{\gamma\}$ properly and is such that $\operatorname{disk}\left(\gamma_{l}\right) \cap S=T$.

Let $\gamma^{\prime \prime}$ be a curve of $\mathcal{C} \backslash\left\{\gamma, \gamma^{\prime}\right\}$ that intersects $l$ at a point $p$. Since $b=\left(\operatorname{disk}(\gamma) \backslash \overline{\operatorname{disk}\left(\gamma_{l}\right)}\right) \backslash \bar{a}$ does not contain any vertex of $\gamma^{\prime \prime}$, the edge of $\gamma^{\prime \prime}$ that contains $p$ and cuts $b$ intersects again $\partial b$ at a point $q \neq p$. Since $\gamma^{\prime \prime}$ does not intersect $\partial b \cap \gamma, q \in \gamma^{\prime} \cap \partial a$. Then, the curve $\gamma^{\prime \prime}$ intersects again $\partial a$ at a point $r \neq q$ such that the arc of $\gamma^{\prime \prime}$ that connects $q$ and $r$ and does not contain $p$ is contained in $a$. The point $r$ cannot belong to $\gamma^{\prime}$, since otherwise one of the connected components of $\operatorname{disk}\left(\gamma^{\prime \prime}\right) \backslash \overline{\operatorname{disk}\left(\gamma^{\prime}\right)}$ would be strictly contained in $a$, in contradiction with the minimality of the area of $a$. Thus, $r$ is on $l^{\prime}$ and, since all intersections are proper, the number of intersections of $\gamma^{\prime \prime}$ with $\gamma_{l}$ is at most equal to the number of intersections of $\gamma^{\prime \prime}$ with $\gamma$. Furthermore, since $u$ and $v$ are points of $\gamma \cap \gamma^{\prime}$ and not of $\gamma_{l} \cap \gamma^{\prime}$, substituting $\gamma$ by $\gamma_{l}$ in $\mathcal{C}$ decreases by at least two the number of intersections between the curves. This contradicts the minimality of the number of intersections in $\mathcal{C}$ and therefore the assumption $A \neq \emptyset$ is absurd.

(b) Now we prove (i). Let $T$ and $T^{\prime}$ be two convex subsets of $S$ and let $\gamma$ and $\gamma^{\prime}$ be two separating curves of $T$ and $T^{\prime}$ that either are disjoint or intersect properly in a minimum set of points. Two such curves exist from (a).

If $\gamma$ and $\gamma^{\prime}$ are disjoint, either $\operatorname{disk}(\gamma) \cap \operatorname{disk}\left(\gamma^{\prime}\right)=\emptyset$, or $\operatorname{disk}(\gamma) \subseteq \operatorname{disk}\left(\gamma^{\prime}\right)$, or $\operatorname{disk}\left(\gamma^{\prime}\right) \subseteq$ $\operatorname{disk}(\gamma)$. Since $T=\operatorname{disk}(\gamma) \cap S$ and $T^{\prime}=\operatorname{disk}\left(\gamma^{\prime}\right) \cap S$, we have in the three cases $\operatorname{conv}\left(T \backslash T^{\prime}\right) \cap$ $\operatorname{conv}\left(T^{\prime} \backslash T\right)=\emptyset$.

If $\gamma$ and $\gamma^{\prime}$ intersect in two points, let $\Delta$ be the oriented straight line that contains $\gamma \cap \gamma^{\prime}$ such that $\operatorname{disk}(\gamma) \backslash \operatorname{disk}\left(\gamma^{\prime}\right) \subset \Delta^{+}$. Hence, we have $T \backslash T^{\prime}=\left(\operatorname{disk}(\gamma) \backslash \operatorname{disk}\left(\gamma^{\prime}\right)\right) \cap S \subset \Delta^{+}$and $T^{\prime} \backslash T=\left(\operatorname{disk}\left(\gamma^{\prime}\right) \backslash \operatorname{disk}(\gamma)\right) \cap S \subset \Delta^{-}$. Thus, $\operatorname{conv}\left(T \backslash T^{\prime}\right) \cap \operatorname{conv}\left(T^{\prime} \backslash T\right)=\emptyset$.

If $\gamma$ and $\gamma^{\prime}$ intersect in more than two points, then $\left(\operatorname{disk}(\gamma) \backslash \overline{\operatorname{disk}\left(\gamma^{\prime}\right)}\right) \cup\left(\operatorname{disk}\left(\gamma^{\prime}\right) \backslash \overline{\operatorname{disk}(\gamma)}\right)$ 
admits at least four connected components. Let $a_{1}, \ldots, a_{4}$ be four of these components, consecutive around $\gamma$, and such that $a_{1}, a_{3} \subset \operatorname{disk}(\gamma) \backslash \overline{\operatorname{disk}\left(\gamma^{\prime}\right)}$ and $a_{2}, a_{4} \subset \operatorname{disk}\left(\gamma^{\prime}\right) \backslash \overline{\operatorname{disk}(\gamma)}$. From (a), for all $i \in\{1, \ldots, 4\}, a_{i}$ contains a point $x_{i}$ of $S$. Now, $\left[x_{1}, x_{3}\right] \cap\left[x_{2}, x_{4}\right] \neq \emptyset$ and, since $x_{1}, x_{3} \in T \backslash T^{\prime}$ and $x_{2}, x_{4} \in T^{\prime} \backslash T$, it follows that $\operatorname{conv}\left(T \backslash T^{\prime}\right) \cap \operatorname{conv}\left(T^{\prime} \backslash T\right) \neq \emptyset$.

Finally, $\operatorname{conv}\left(T \backslash T^{\prime}\right) \cap \operatorname{conv}\left(T^{\prime} \backslash T\right)=\emptyset$ if and only if $T$ and $T^{\prime}$ are compatible.

(c) At last, we prove (ii). Let $\mathcal{F}$ be a family of subsets of $S$ separable by a set $\mathcal{C}$ of convex pseudo-circles. Since the curves in $\mathcal{C}$ are convex and pairwise compatible, the subsets in $\mathcal{F}$ are also convex and pairwise compatible.

The converse is by contrapositive. Let $\mathcal{F}$ be a set of convex subsets of $S$ and let $\mathcal{C}$ be a set of separating curves of $\mathcal{F}$ such that the intersections of the curves in $\mathcal{C}$ are proper and their number is minimal. If two curves $\gamma$ and $\gamma^{\prime}$ of $\mathcal{C}$ intersect more than twice then, as in proof of (b), the subsets $T$ and $T^{\prime}$ in $\mathcal{F}$ separable by $\gamma$ and $\gamma^{\prime}$ are incompatible. It follows that, if the subsets in $\mathcal{F}$ are pairwise compatible, $\mathcal{F}$ is separable by convex pseudo-circles.

In the remainder of the paper, $\mathcal{F}$ denotes a maximal (with respect to inclusion) family of subsets of $S$ separable by convex pseudo-circles, i.e., from the previous proposition, a maximal family of compatible convex subsets of $S$. Note that $\emptyset$ and $S$ are always in $\mathcal{F}$.

\subsection{Convex pairs}

It is often more convenient to handle separating lines that pass through points of $S$ rather than strictly separating lines. For example, it is very easy to enumerate the total number of subsets of $S$ separable by straight lines that pass through two points of $S$. In the same way, to compute the number of subsets strictly separable by circles, Lee [9] established relations between this number and the numbers of subsets separable by circles passing through two or three points.

Even if convex pseudo-circles have a greater degree of freedom (a single convex pseudo-circle is an arbitrary convex curve), we will see in this section that only those passing through at most three points of $S$ are compatible with a maximal family of strictly separating convex pseudocircles. In Section 3, convex pseudo-circles passing through points of $S$ will be necessary to characterize the edges of a graph whose vertices are subsets of $S$ strictly separable by convex pseudo-circles. Therefore, we now study pairs $(P, Q)$ of subsets of $S$ for which there exist convex Jordan curves $\gamma$ such that $\gamma \cap S=Q$ and $\operatorname{disk}(\gamma) \cap S=P$. Again, we prefer to characterize these pairs with convex hulls instead of curves.

Definition 2.5. An ordered pair $(P, Q)$ of disjoint subsets of $S$ is called a convex pair if $\operatorname{conv}(P \cup Q) \cap S=P \cup Q$ and if $Q$ is a subset of the extreme points of $P \cup Q$.

The proof that both definitions are equivalent is a straightforward extention of proof of Proposition 2.2. Actually, we will not explicitly use the characterization of convex pairs with curves to prove the results in this paper, but it helps intuition about them.

In the same way, a proof similar to that of Proposition 2.4 shows that the following definition characterizes convex pairs that are determined by two compatible convex Jordan curves; See also Figure 3 (a).

Definition 2.6. Two convex pairs $(P, Q)$ and $\left(P^{\prime}, Q^{\prime}\right)$ are said to be compatible if

$$
\operatorname{relint}\left(\operatorname{conv}\left((P \cup Q) \backslash P^{\prime}\right)\right) \cap \operatorname{relint}\left(\operatorname{conv}\left(\left(P^{\prime} \cup Q^{\prime}\right) \backslash P\right)\right)=\emptyset .
$$

The use of relative interiors is necessary to prove that Definition 2.6 is equivalent to saying that the two convex pairs $(P, Q)$ and $\left(P^{\prime}, Q^{\prime}\right)$ are determined by compatible convex Jordan curves. Indeed, if $Q \cap Q^{\prime} \neq \emptyset$, then $\operatorname{conv}\left((P \cup Q) \backslash P^{\prime}\right) \cap \operatorname{conv}\left(\left(P^{\prime} \cup Q^{\prime}\right) \backslash P\right) \neq \emptyset$ although the pairs $(P, Q)$ and $\left(P^{\prime}, Q^{\prime}\right)$ may be separable by compatible curves; See Figure 3 (b).

Conversely, the following lemma shows that one can drop the relative interiors when $Q \cap Q^{\prime}=$ $\emptyset$. Coupled with Lemma 2.10, this simplifies most proofs of the forthcoming results. 


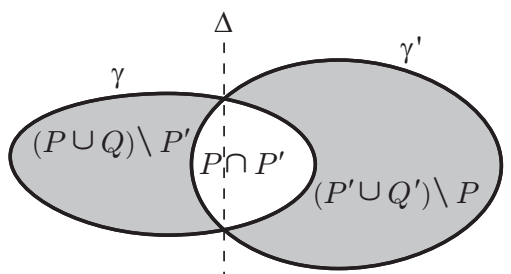

(a)

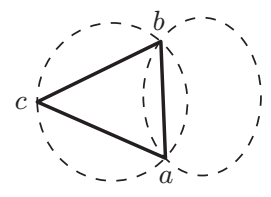

(b)

Figure 3: (a) If the convex pairs $(P, Q)$ and $\left(P^{\prime}, Q^{\prime}\right)$ are determined by two properly intersecting convex pseudo-circles $\gamma$ and $\gamma^{\prime}$, then $(P \cup Q) \backslash P^{\prime}$ and $\left(P^{\prime} \cup Q^{\prime}\right) \backslash P$ are on both sides of (or on) the straight line $\Delta$ containing $\gamma \cap \gamma^{\prime}$. The points of these subsets that are on $\Delta$ are the points of $Q \cap Q^{\prime}$. The set $P \cap P^{\prime}$ is in the open area $\operatorname{disk}(\gamma) \cap \operatorname{disk}\left(\gamma^{\prime}\right)$. (b) The pairs $(\emptyset,\{a, b, c\})$ and $(\emptyset,\{a, b\})$ are compatible, although the closed triangle $[a, b, c]$ and the closed segment $[a, b]$ intersect.

Lemma 2.7. If two distinct convex pairs $(P, Q)$ and $\left(P^{\prime}, Q^{\prime}\right)$ are compatible and if $Q \cap Q^{\prime}=\emptyset$, then $\operatorname{conv}\left((P \cup Q) \backslash P^{\prime}\right) \cap \operatorname{conv}\left(\left(P^{\prime} \cup Q^{\prime}\right) \backslash P\right)=\emptyset$.

Proof. If the convexes $\operatorname{conv}\left((P \cup Q) \backslash P^{\prime}\right)$ and $\operatorname{conv}\left(\left(P^{\prime} \cup Q^{\prime}\right) \backslash P\right)$ intersect while their relative interiors are disjoint, these two convexes have a common vertex or a common edge, since $S$ contains no three collinear points. Now, the only possible common points of $(P \cup Q) \backslash P^{\prime}$ and $\left(P^{\prime} \cup Q^{\prime}\right) \backslash P$ are the points of $Q \cap Q^{\prime}$.

This lemma also shows that Definition 2.6 is consistent with Proposition 2.4 (i). Indeed, from Proposition 2.2, $(T, \emptyset)$ is a convex pair of $S$ if and only if $T$ is a convex subset of $S$. From Lemma 2.7, two convex pairs $(T, \emptyset)$ and $\left(T^{\prime}, \emptyset\right)$ are compatible if and only if the convex subsets $T$ and $T^{\prime}$ are compatible.

Notice also that a convex pair $(P, Q)$ is compatible with itself if and only if $Q=\emptyset$.

We now state inclusion relations between convex pairs.

Lemma 2.8. (i) If $(P, Q)$ is a convex pair of $S$ then every intermediate pair $\left(P^{\prime}, Q^{\prime}\right)$ of disjoint subsets of $S$, i.e. such that $P \subseteq P^{\prime} \subseteq P^{\prime} \cup Q^{\prime} \subseteq P \cup Q$, is also a convex pair of $S$.

(ii) Furthermore, if $|Q| \leq 3$ and $\left(P^{\prime}, Q^{\prime}\right) \neq(P, Q)$, then $\left(P^{\prime}, Q^{\prime}\right)$ is compatible with $(P, Q)$.

Proof. (i) Since $\operatorname{conv}(P \cup Q) \cap S=P \cup Q$, one has $\operatorname{conv}\left(P^{\prime} \cup Q^{\prime}\right) \cap S \subseteq P \cup Q$. Now, every point of $P \cup Q$ not in $P^{\prime} \cup Q^{\prime}$ is a point of $Q$, that is, an extreme point of $P \cup Q$. Hence, this point does not belong to $\operatorname{conv}\left(P^{\prime} \cup Q^{\prime}\right)$, and $\operatorname{conv}\left(P^{\prime} \cup Q^{\prime}\right) \cap S=P^{\prime} \cup Q^{\prime}$. Since the points of $Q^{\prime}$ are points of $Q$, they are extreme points of $P \cup Q$, hence of $P^{\prime} \cup Q^{\prime}$. Therefore, $\left(P^{\prime}, Q^{\prime}\right)$ is a convex pair of $S$.

(ii) The sets $(P \cup Q) \backslash P^{\prime}$ and $\left(P^{\prime} \cup Q^{\prime}\right) \backslash P$ are contained in $Q$ and, if $|Q| \leq 3$, they are vertices of a same triangle. Therefore, $\operatorname{relint}\left(\operatorname{conv}\left((P \cup Q) \backslash P^{\prime}\right)\right)$ and $\operatorname{relint}\left(\operatorname{conv}\left(\left(P^{\prime} \cup Q^{\prime}\right) \backslash P\right)\right)$ can only intersect if $(P \cup Q) \backslash P^{\prime}=\left(P^{\prime} \cup Q^{\prime}\right) \backslash P$, i.e., if $P^{\prime}=P$ and $Q^{\prime}=Q$, in contradiction with the hypothesis.

Definition 2.9. We call subset of a convex pair $(P, Q)$ any set $T$ such that $P \subseteq T \subseteq P \cup Q$.

It follows from the previous lemma that a subset of a convex pair is a convex subset. The next lemma shows that compatibility between convex pairs reduces to compatibility between convex subsets.

Lemma 2.10. Two distinct convex pairs $(P, Q)$ and $\left(P^{\prime}, Q^{\prime}\right)$ are compatible if and only if the subsets of $(P, Q)$ are compatible with the subsets of $\left(P^{\prime}, Q^{\prime}\right)$. 
Proof. (a) If $(P, Q)$ and $\left(P^{\prime}, Q^{\prime}\right)$ are compatible,

$$
\operatorname{relint}\left(\operatorname{conv}\left((P \cup Q) \backslash P^{\prime}\right)\right) \cap \operatorname{relint}\left(\operatorname{conv}\left(\left(P^{\prime} \cup Q^{\prime}\right) \backslash P\right)\right)=\emptyset .
$$

If a subset $T$ of $(P, Q)$ is incompatible with a subset $T^{\prime}$ of $\left(P^{\prime}, Q^{\prime}\right)$, the pairs $(T, \emptyset)$ and $\left(T^{\prime}, \emptyset\right)$ are also incompatible from Lemma 2.7 and, therefore, $\operatorname{relint}\left(\operatorname{conv}\left(T \backslash T^{\prime}\right)\right) \cap \operatorname{relint}\left(\operatorname{conv}\left(T^{\prime} \backslash T\right)\right) \neq \emptyset$. Now, $T \backslash T^{\prime} \subseteq(P \cup Q) \backslash P^{\prime}$ and $T^{\prime} \backslash T \subseteq\left(P^{\prime} \cup Q^{\prime}\right) \backslash P$. Thus, the relative interiors of $\operatorname{conv}\left(T \backslash T^{\prime}\right)$ and of $\operatorname{conv}\left(T^{\prime} \backslash T\right)$ can only intersect if they are contained in the common boundary of the two convexes $\operatorname{conv}\left((P \cup Q) \backslash P^{\prime}\right)$ and $\operatorname{conv}\left(\left(P^{\prime} \cup Q^{\prime}\right) \backslash P\right)$. Since $S$ has no three collinear points, $\operatorname{conv}\left(T \backslash T^{\prime}\right)$ and $\operatorname{conv}\left(T^{\prime} \backslash T\right)$ are then a common vertex or a common edge of these two convexes. Hence, $T \backslash T^{\prime}=T^{\prime} \backslash T$, i.e., $T=T^{\prime}$, in contradiction with the hypothesis $\operatorname{relint}\left(\operatorname{conv}\left(T \backslash T^{\prime}\right)\right) \cap \operatorname{relint}\left(\operatorname{conv}\left(T^{\prime} \backslash T\right)\right) \neq \emptyset$.

(b) The proof of the converse is by contrapositive.

(b.1) We show first that, for every finite subset $A$ with at least two elements, if $x \in$ $\operatorname{relint}(\operatorname{conv}(A))$ then there exists a family $\left(\lambda_{a}\right)_{a \in A}$ of numbers in $] 0,1[$ with sum 1 such that $x=\sum_{a \in A} \lambda_{a} a$.

Let $g=\frac{1}{|A|} \sum_{a \in A} a$ be the centroid of $A$. For $t>1$ close enough to 1 , the point $x+(t-$ $1)(x-g)$ belongs to $\operatorname{conv}(A)$ because $x$ is in the relative interior of $\operatorname{conv}(A)$. Consequently, there exists a family $\left(\mu_{a}\right)_{a \in A}$ of numbers $\in[0,1]$ with sum 1 such that $g+t(x-g)=\sum_{a \in A} \mu_{a} a$. It follows that $x=\frac{1}{t}\left(\sum_{a \in A} \mu_{a} a+(t-1) g\right)$. This gives the family $\lambda_{a}=\frac{1}{t}\left(\mu_{a}+\frac{t-1}{|A|}\right)$.

(b.2) If $(P, Q)$ and $\left(P^{\prime}, Q^{\prime}\right)$ are incompatible, then the relative interiors of the convexes $\operatorname{conv}\left((P \cup Q) \backslash P^{\prime}\right)$ and $\operatorname{conv}\left(\left(P^{\prime} \cup Q^{\prime}\right) \backslash P\right)$ intersect. Thus, from (ii.1) and taking into account that both $(P \cup Q) \backslash P^{\prime}$ and $\left(P^{\prime} \cup Q^{\prime}\right) \backslash P$ can be reduced to a point, there exist two families $\left(\lambda_{u}\right)_{u \in(P \cup Q) \backslash P^{\prime}}$ and $\left(\mu_{v}\right)_{v \in\left(P^{\prime} \cup Q^{\prime}\right) \backslash P}$ of numbers $\left.\left.\in\right] 0,1\right]$ with sum 1 such that

$$
\sum_{u \in(P \cup Q) \backslash P^{\prime}} \lambda_{u} u=\sum_{v \in\left(P^{\prime} \cup Q^{\prime}\right) \backslash P} \mu_{v} v .
$$

Setting $W=\left((P \cup Q) \backslash P^{\prime}\right) \cap\left(\left(P^{\prime} \cup Q^{\prime}\right) \backslash P\right)=Q \cap Q^{\prime}$, this relation becomes

$$
\sum_{u \in(P \cup Q) \backslash\left(P^{\prime} \cup W\right)} \lambda_{u} u+\sum_{w \in W} \lambda_{w} w=\sum_{v \in\left(P^{\prime} \cup Q^{\prime}\right) \backslash(P \cup W)} \mu_{v} v+\sum_{w \in W} \mu_{w} w .
$$

In order to use compatibility of subsets of $(P, Q)$ and $\left(P^{\prime}, Q^{\prime}\right)$, we transform the preceding relation in a relation between two centroids associated with disjoint sets of points. To this aim, we split $W$ in three subsets:

$$
\begin{aligned}
& W_{Q}=\left\{w \in W: \lambda_{w}>\mu_{w}\right\} \\
& W_{Q^{\prime}}=\left\{w \in W: \lambda_{w}<\mu_{w}\right\} \\
& W_{0}=\left\{w \in W: \lambda_{w}=\mu_{w}\right\} .
\end{aligned}
$$

The terms of the relation associated with elements of $W_{0}$ are eliminated and we get

$$
\sum_{u \in(P \cup Q) \backslash\left(P^{\prime} \cup W\right)} \lambda_{u} u+\sum_{w \in W_{Q}}\left(\lambda_{w}-\mu_{w}\right) w \quad \sum_{v \in\left(P^{\prime} \cup Q^{\prime}\right) \backslash(P \cup W)} \mu_{v} v+\sum_{w \in W_{Q^{\prime}}}\left(\mu_{w}-\lambda_{w}\right) w .
$$

Clearly, from the first equality to the third one, the sum of the coefficients in the left member remains equal to the sum of the coefficients in the right member. By construction, these coefficients are all positive. Furthermore, $(P, Q) \neq\left(P^{\prime}, Q^{\prime}\right)$ implies that we cannot have $(P \cup Q) \backslash\left(P^{\prime} \cup W\right)=\left(P^{\prime} \cup Q^{\prime}\right) \backslash(P \cup W)=\emptyset$. Indeed, since $W=Q \cap Q^{\prime}$ and $P \cap Q=\emptyset$, if $(P \cup Q) \backslash\left(P^{\prime} \cup W\right)=\emptyset$ we have $P \subseteq P^{\prime}$. In the same way, $\left(P^{\prime} \cup Q^{\prime}\right) \backslash(P \cup W)=\emptyset$ implies $P^{\prime} \subseteq P$ and, therefore, $P^{\prime}=P$. We then also have $Q \subseteq Q \cap Q^{\prime}$ and $Q^{\prime} \subseteq Q \cap Q^{\prime}$, i.e., $Q=Q^{\prime}$. 
Hence, the last equality can be divided by the sum of the coefficients of one of its members, and we get a point of the intersection

$$
\operatorname{conv}\left(\left((P \cup Q) \backslash\left(P^{\prime} \cup W\right)\right) \cup W_{Q}\right) \cap \operatorname{conv}\left(\left(\left(P^{\prime} \cup Q^{\prime}\right) \backslash(P \cup W)\right) \cup W_{Q^{\prime}}\right) .
$$

Set

$$
\begin{aligned}
& A=P \cup\left((P \cup Q) \backslash\left(P^{\prime} \cup W\right)\right) \cup W_{Q}, \\
& B=P^{\prime} \cup\left(\left(P^{\prime} \cup Q^{\prime}\right) \backslash(P \cup W)\right) \cup W_{Q^{\prime}} .
\end{aligned}
$$

Since $P \subseteq A \subseteq P \cup Q$ and $P^{\prime} \subseteq B \subseteq P^{\prime} \cup Q^{\prime}, A$ and $B$ are respective subsets of $(P, Q)$ and of $\left(P^{\prime}, Q^{\prime}\right)$. Now,

$$
\begin{gathered}
\left((P \cup Q) \backslash\left(P^{\prime} \cup W\right)\right) \cup W_{Q} \subseteq A \backslash B, \\
\left(\left(P^{\prime} \cup Q^{\prime}\right) \backslash(P \cup W)\right) \cup W_{Q^{\prime}} \subseteq B \backslash A .
\end{gathered}
$$

Consequently, the two subsets $A$ and $B$ are incompatible.

In the remainder, we will be particularly interested in convex pairs that are compatible with the maximal family $\mathcal{F}$ of compatible convex subsets. We will say that a convex pair $(P, Q)$ is compatible with $\mathcal{F}$ if $(P, Q)$ is compatible with all pairs $(T, \emptyset)$ such that $T \in \mathcal{F}$. Since $\mathcal{F}$ is maximal, $(P, \emptyset)$ compatible with $\mathcal{F}$ is equivalent to $P \in \mathcal{F}$. More generally, we have:

Proposition 2.11. (i) A convex pair of $S$ is compatible with $\mathcal{F}$ if and only if its subsets are in $\mathcal{F}$.

(ii) Two distinct convex pairs of $S$ that are compatible with $\mathcal{F}$ are compatible with one another.

(iii) If $(P, Q)$ is compatible with $\mathcal{F}$ then $|Q| \leq 3$.

Proof. (i) From Lemma 2.10, a convex pair $(P, Q)$ is compatible with every subset $T$ in $\mathcal{F}$ if and only if the subsets of $(P, Q)$ are also compatible with every $T$ in $\mathcal{F}$, i.e. if and only if these subsets are in $\mathcal{F}$, since $\mathcal{F}$ is maximal.

(ii) From (i), the subsets of two convex pairs that are compatible with $\mathcal{F}$ are in $\mathcal{F}$ and thus, they are compatible with each others. Hence, from Lemma 2.10, the two convex pairs are compatible with each other, if they are distinct.

(iii) Let $(P, Q)$ be a convex pair with $|Q| \geq 4$. Since $Q$ is in convex position, $Q$ admits four extreme points $q_{1}, \ldots, q_{4}$ consecutive in this order on the boundary of $\operatorname{conv}(Q)$, and then $\left[q_{1}, q_{3}\right] \cap\left[q_{2}, q_{4}\right] \neq \emptyset$. Now, $\left\{q_{1}, q_{3}\right\}=\left(P \cup\left\{q_{1}, q_{3}\right\}\right) \backslash\left(P \cup\left\{q_{2}, q_{4}\right\}\right)$ and $\left\{q_{2}, q_{4}\right\}=\left(P \cup\left\{q_{2}, q_{4}\right\}\right) \backslash$ $\left(P \cup\left\{q_{1}, q_{3}\right\}\right)$. Thus, the subsets $P \cup\left\{q_{1}, q_{3}\right\}$ and $P \cup\left\{q_{2}, q_{4}\right\}$ of $(P, Q)$ are incompatible with each other and cannot be simultaneously in $\mathcal{F}$. From $(\mathrm{i}),(P, Q)$ is then incompatible with $\mathcal{F}$.

\section{Order- $k$ centroid triangulations}

Given an integer $k \in\{1, \ldots, n-1\}$, the $k$-subsets of $S$ separable by circles can be enumerated by studying the adjacency relations between regions, edges, and vertices of the order- $k$ Voronoi diagram of $S$ [9]. Recall that this diagram is a partition of the plane, each region of which is associated with a $k$-subset $T$ of $S$ separable by a circle. More precisely, the order- $k$ Voronoi region of $T$ is the set of centers of all circles that separate $T$ from $S$ (see Figure 4). The edges of the order- $k$ Voronoi diagram are the sets of centers of the circles passing through two points of $S$ and that separate $k-1$ points. When $S$ has no four cocircular points, the vertices of the diagram are the centers of the circles passing through three points of $S$ and that separate either $k-1$ or $k-2$ points of $S$.

The order- $k$ Voronoi diagram of $S$ admits a dual graph whose vertices are the centroids of the $k$-subsets of $S$ separable by circles $[3,15]$. When $S$ has no four cocircular points, this dual graph is a triangulation called the order- $k$ (centroid) Delaunay triangulation of $S$. 


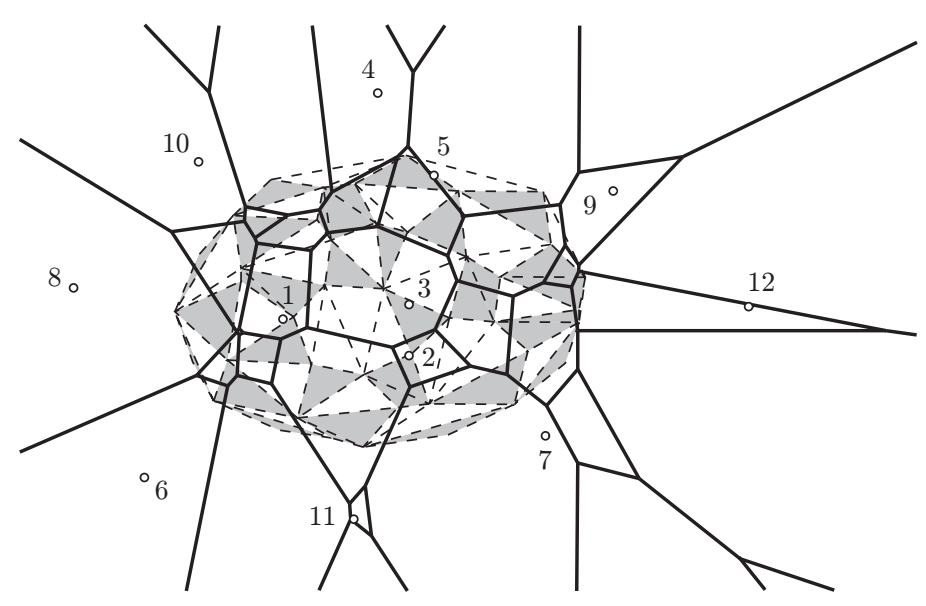

Figure 4: The order-4 Voronoi diagram (full lines) and the order-4 centroid Delaunay triangulation (dashed lines) of 12 points in the plane. White triangles are type- 1 and gray triangles are type-2 (see Observation 3.2).

In this section, we construct analogous centroid triangulations from families of $k$-subsets of $S$ separable by convex pseudo-circles.

For every integer $k$ in $\{1, \ldots, n\}$, let $\mathcal{F}_{k}$ be the set of elements of size $k$ in a maximal family $\mathcal{F}$ of subsets of $S$ separable by convex pseudo-circles. We will prove later that $\mathcal{F}_{k}$ is maximal among all the $k$-subsets of $S$ separable by convex pseudo-circles.

We want to build a triangulation of the centroids of the elements of $\mathcal{F}_{k}$. In order to characterize the edges and triangles of this triangulation, we focus on the convex pairs compatible with $\mathcal{F}$ that admit subsets in $\mathcal{F}_{k}$. For every such pair $(P, Q)$, one has $|P| \leq k \leq|P \cup Q|$ and, from Proposition 2.11, $|Q| \leq 3$. Furthermore, if $|P|=k,(P, Q)$ has $P$ as unique $k$-subset, and if $|P \cup Q|=k,(P, Q)$ has $P \cup Q$ as unique $k$-subset. Among the pairs $(P, Q)$ such that $|P|=k$ or $|P \cup Q|=k$, we restrict ourselves to those for which $Q=\emptyset$. This explains the following definition of convex $k$-pairs.

Definition 3.1. (i) A convex pair $(P, Q)$ of $S$ is called a convex $k$-pair if

- either $|P|=k$ and $Q=\emptyset$,

- or $|P|<k<|P \cup Q|$ and $2 \leq|Q| \leq 3$.

(ii) For a convex $k$-pair $(P, Q)$, the convex hull of the set of centroids of all $k$-subsets of $(P, Q)$, is called the $k$-set polygon of $(P, Q)$, denoted by $\mathcal{Q}_{k}(P, Q)$.

Notice that, in the case $P=\emptyset$, the $k$-set polygon of $(P, Q)$ is nothing but the $k$-set polygon of $Q$, defined for point sets by Edelsbrunner, Valtr, and Welzl [7] (see also later in this section).

Observation 3.2. Let us describe all types of convex $k$-pairs $(P, Q)$ and their $k$-set polygons.

- When $Q=\emptyset, \mathcal{Q}_{k}(P, Q)$ is the centroid $g(P)$ of the convex $k$-subset $P$.

- When $Q=\{s, t\}$, one has $|P|=k-1$, and $\mathcal{Q}_{k}(P, Q)$ is the segment $[g(P \cup\{s\}), g(P \cup\{t\})]$. From Lemma 2.8, $P \cup\{s\}$ and $P \cup\{t\}$ are convex $k$-subsets compatible with $(P, Q)$.

- When $Q=\{r, s, t\}$ and $|P|=k-1, \mathcal{Q}_{k}(P, Q)$ is the triangle with vertices the centroids of the convex $k$-subsets $P \cup\{r\}, P \cup\{s\}, P \cup\{t\}$; Such a triangle is said of type 1. From Lemma 2.8, the edges $[g(P \cup\{r\}), g(P \cup\{s\})],[g(P \cup\{s\}), g(P \cup\{t\})],[g(P \cup\{t\}), g(P \cup\{r\})]$ of this triangle are the $k$-set polygons of the convex $k$-pairs $(P,\{r, s\}),(P,\{s, t\}),(P,\{t, r\})$, and these pairs are compatible with $(P, Q)$; See Figure 5(a). 
- When $Q=\{r, s, t\}$ and $|P|=k-2, \mathcal{Q}_{k}(P, Q)$ is the triangle with vertices the centroids of the convex $k$-subsets $P \cup\{r, s\}, P \cup\{s, t\}, P \cup\{t, r\}$; Such a triangle is said of type 2 . Its edges are the $k$-set polygons of the convex $k$-pairs $(P \cup\{s\},\{r, t\}),(P \cup\{t\},\{s, r\})$, and $(P \cup\{r\},\{s, t\})$, which are compatible with $(P, Q)$; See Figure 5(b).

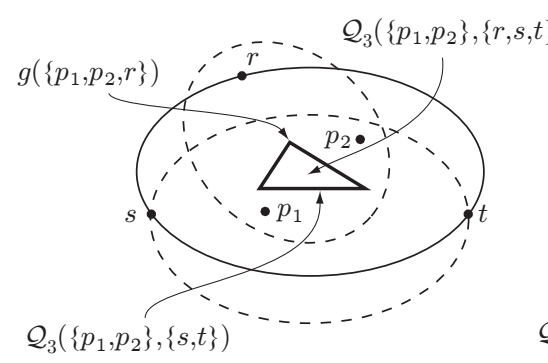

(a)

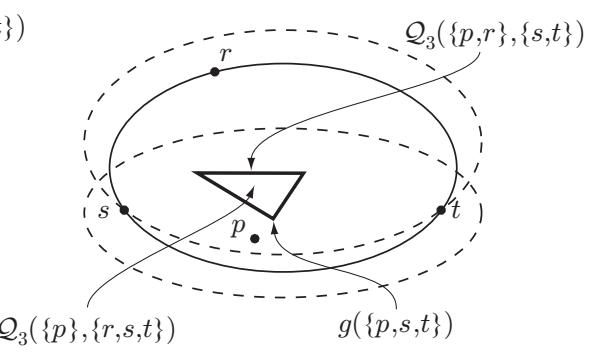

(b)

Figure 5: The 3-set polygons of the convex 3-pairs $(P,\{r, s, t\})$ with $P=\left\{p_{1}, p_{2}\right\}$ (a) and with $P=\{p\}$ (b) are respectively type- 1 and type- 2 triangles.

Together with Proposition 2.11 (i), this observations lead to the following proposition.

Proposition 3.3. If $(P, Q)$ is a convex $k$-pair compatible with $\mathcal{F}$ such that $Q \neq \emptyset$, then the vertices of $\mathcal{Q}_{k}(P, Q)$, as well as its edges if it is a triangle, are $k$-set polygons of convex $k$-pairs compatible with $\mathcal{F}$.

Proof. It follows from Observation 3.2 that every vertex and, where appropriate, every edge of the $k$-set polygon of a convex $k$-pair $(P, Q)$ with $Q \neq \emptyset$ is the $k$-set polygon of a convex $k$-pair $\left(P^{\prime}, Q^{\prime}\right) \neq(P, Q)$ such that $P \subseteq P^{\prime}$ and $P^{\prime} \cup Q^{\prime} \subseteq P \cup Q$. Thus, the subsets of $\left(P^{\prime}, Q^{\prime}\right)$ are also subsets of $(P, Q)$. From Proposition $2.11(\mathrm{i})$, if $(P, Q)$ is compatible with $\mathcal{F}$, its subsets are in $\mathcal{F}$. It is the same with the subsets of $\left(P^{\prime}, Q^{\prime}\right)$ and, still from Proposition 2.11 (i), $\left(P^{\prime}, Q^{\prime}\right)$ is compatible with $\mathcal{F}$.

Using basic properties of centroids, we show that the $k$-set polygons of convex $k$-pairs compatible with $\mathcal{F}$ (hence compatible with each others from Proposition 2.11 (ii)) have disjoint relative interiors.

Proposition 3.4. If $(P, Q)$ and $\left(P^{\prime}, Q^{\prime}\right)$ are two distinct compatible convex $k$-pairs, then

$$
\operatorname{relint}\left(\mathcal{Q}_{k}(P, Q)\right) \cap \operatorname{relint}\left(\mathcal{Q}_{k}\left(P^{\prime}, Q^{\prime}\right)\right)=\emptyset .
$$

Proof. Since relint $\left(\operatorname{conv}\left((P \cup Q) \backslash P^{\prime}\right)\right) \cap \operatorname{relint}\left(\operatorname{conv}\left(\left(P^{\prime} \cup Q^{\prime}\right) \backslash P\right)\right)=\emptyset$, there exists an oriented straight line $\Delta$ such that $(P \cup Q) \backslash P^{\prime} \subset \overline{\Delta^{+}},\left(P^{\prime} \cup Q^{\prime}\right) \backslash P \subset \overline{\Delta^{-}}$and, within a permutation of $(P, Q)$ and $\left(P^{\prime}, Q^{\prime}\right),\left(P^{\prime} \cup Q^{\prime}\right) \backslash P \not \subset \Delta$.

Let $T$ be a $k$-subset of $(P, Q)$ such that $g(T)$ is a vertex of $\mathcal{Q}_{k}(P, Q)$ that belongs to a straight line $\Delta^{\prime}$ parallel to $\Delta$, oriented in the same direction as $\Delta$, and such that $\mathcal{Q}_{k}(P, Q) \subset \overline{\Delta^{\prime+}}$. For every $k$-subset $T^{\prime} \neq T$ of $\left(P^{\prime}, Q^{\prime}\right)$, the sets $A=T \backslash T^{\prime}$ and $B=T^{\prime} \backslash T$ are nonempty and are respectively contained in $(P \cup Q) \backslash P^{\prime}$ and in $\left(P^{\prime} \cup Q^{\prime}\right) \backslash P$. Since the vectors $g(A) g(B)$ and $g(T) g\left(T^{\prime}\right)=g\left(\left(T \cap T^{\prime}\right) \cup A\right) g\left(\left(T \cap T^{\prime}\right) \cup B\right)$ have same direction, we have $g\left(T^{\prime}\right) \in \overline{\Delta^{\prime-}}$. Hence, $\mathcal{Q}_{k}\left(P^{\prime}, Q^{\prime}\right) \subset \overline{\Delta^{\prime-}}$. Furthermore, if $T^{\prime}$ contains a point of $\left(\left(P^{\prime} \cup Q^{\prime}\right) \backslash P\right) \cap \Delta^{-}, g(A) \in \Delta^{-}$and $g\left(T^{\prime}\right) \in \Delta^{\prime-}$. Since $\left(P^{\prime} \cup Q^{\prime}\right) \backslash P \not \subset \Delta$, such a subset $T^{\prime}$ exists and, therefore, $\operatorname{relint}\left(\mathcal{Q}_{k}\left(P^{\prime}, Q^{\prime}\right)\right) \subset$ $\Delta^{\prime-}$. Hence, $\operatorname{relint}\left(\mathcal{Q}_{k}(P, Q)\right) \cap \operatorname{relint}\left(\mathcal{Q}_{k}\left(P^{\prime}, Q^{\prime}\right)\right)=\emptyset$.

The two propositions above show that every set of $k$-set polygons of convex $k$-pairs compatible with $\mathcal{F}$ forms a simplicial complex. We show now that, if this set is maximal, it forms 
a triangulation. For this, we first show that the convex hull of the centroids of the elements in $\mathcal{F}_{k}$ is the $k$-set polygon of $S$.

Recall that the $k$-set polygon of $S$, denoted by $\mathcal{Q}_{k}(S)$, is the convex hull of the centroids of all $k$-point subsets of $S$ [7]. Note that $\mathcal{Q}_{1}(S)$ is simply the convex hull of $S$.

The characterizations of the vertices and of the edges of $\mathcal{Q}_{k}(S)$ stated in Proposition 3.5 are due to Andrzejak and Fukuda [1], and to Andrzejak and Welzl [2]. Recall for this that a $k$-set of $S$ is a subset of $k$ points of $S$ strictly separable from the others by a straight line.

Proposition 3.5. (i) The centroid $g(T)$ of a subset $T$ of $S$ is a vertex of $\mathcal{Q}_{k}(S)$ if and only if $T$ is a $k$-set of $S$.

(ii) The centroid $g(T)$ is the predecessor of the centroid $g\left(T^{\prime}\right)$ in counterclockwise order on $\partial \mathcal{Q}_{k}(S)$ if and only if there exist $s, t \in S$ such that, setting $P=(s t)^{-} \cap S$, one has $|P|=k-1$, $T=P \cup\{s\}$, and $T^{\prime}=P \cup\{t\}$.

The next proposition shows that the vertices and edges of $\mathcal{Q}_{k}(S)$ are $k$-set polygons of convex $k$-subsets and $k$-pairs of $S$, that are compatible with every maximal family $\mathcal{F}$ separable by convex pseudo-circles.

Proposition 3.6. (i) The $k$-sets of $S$ are in $\mathcal{F}$.

(ii) For every pair $(s, t)$ of points of $S$ such that $\left|(s t)^{-} \cap S\right|=k-1$, the pair $\left((s t)^{-} \cap S,\{s, t\}\right)$ is a convex $k$-pair of $S$ compatible with $\mathcal{F}$.

Proof. We first prove (ii). Setting $P=(s t)^{-} \cap S$, we have $\operatorname{conv}(P \cup\{s, t\}) \cap S=P \cup\{s, t\}$, $s$ and $t$ are extreme points of $P \cup\{s, t\}$, and $|P|<k<|P \cup\{s, t\}|$. Therefore, $(P,\{s, t\})$ is a convex $k$-pair of $S$.

For every convex subset $T$ of $\mathcal{F}$, we have $(P \cup\{s, t\}) \backslash T \subset \overline{(s t)^{-}}$and $T \backslash P \subset \overline{(s t)^{+}}$. Since $(s t) \cap S=\{s, t\}$ and since neither $s$ nor $t$ can be simultaneously in $(P \cup\{s, t\}) \backslash T$ and in $T \backslash P$, $\operatorname{conv}((P \cup\{s, t\}) \backslash T)$ and $\operatorname{conv}(T \backslash P)$ cannot intersect. It follows that $(P,\{s, t\})$ is compatible with $T$.

Now we prove (i). From Proposition 3.5, for every $k$-set $T$ of $S, g(T)$ is an endpoint of an edge of $\mathcal{Q}_{k}(S)$ and, therefore, there exist $s, t \in S$ such that $T=\left((s t)^{-} \cap S\right) \cup\{s\}$. From (ii), $\left((s t)^{-} \cap S,\{s, t\}\right)$ is a convex pair of $S$ compatible with $\mathcal{F}$. Since $T$ is a subset of this pair, $T$ is in $\mathcal{F}$, from Proposition 2.11 (i).

In order to show that the $k$-set polygons of the convex $k$-pairs compatible with $\mathcal{F}$ cover $\mathcal{Q}_{k}(S)$, we need to show the following: For every edge $[g(P \cup\{s\}), g(P \cup\{t\})]$ of the simplicial complex determined by Propositions 3.3 and 3.4, if $(g(P \cup\{s\}) g(P \cup\{t\}))^{+} \cap \mathcal{Q}_{k}(S) \neq \emptyset$ then there exists a triangle of the simplicial complex in $(g(P \cup\{s\}) g(P \cup\{t\}))^{+}$admitting $[g(P \cup\{s\}), g(P \cup\{t\})]$ as an edge. This comes down to showing the following key result.

Theorem 3.7. For every convex pair $(P,\{s, t\})$ of $S$ compatible with $\mathcal{F}$ such that $(s t)^{+} \cap S \neq P$, there exists a point $r \in\left(P \cap(s t)^{-}\right) \cup\left((S \backslash P) \cap(s t)^{+}\right)$such that $(P \backslash\{r\},\{r, s, t\})$ is a convex pair of $S$ compatible with $\mathcal{F}$.

The long and tricky proof of this theorem is postponed to Section 5. An important consequence of Theorem 3.7 is:

Corollary 3.8. If $e$ is a line segment that is the $k$-set polygon of a convex k-pair of $S$ compatible with $\mathcal{F}$ and if $\mathcal{Q}_{k}(S)$ is not reduced to e, then

- either $e$ is an edge common to two triangles that are the $k$-set polygons of two convex $k$-pairs of $S$ compatible with $\mathcal{F}$, and these two triangles are on both sides of $e$,

- or e is an edge of $\mathcal{Q}_{k}(S)$ and of one triangle that is the $k$-set polygon of a convex $k$-pair of $S$ compatible with $\mathcal{F}$. 
Proof. Let $(P,\{s, t\})$ be the convex $k$-pair of $S$ compatible with $\mathcal{F}$ such that $e=\mathcal{Q}_{k}(P,\{s, t\})$.

(i) If $e$ is an edge of $\mathcal{Q}_{k}(S)$, it follows from Proposition 3.5 that, within a permutation of $s$ and $t,(s t)^{-} \cap S=P$. One cannot have at the same time $(s t)^{+} \cap S=P$, otherwise $P=\emptyset$, $S=\{s, t\}, k=1$, and $\mathcal{Q}_{k}(S)$ would be reduced to the segment $e=[s, t]$. Thus, from Theorem 3.7, there exists $r \in\left(P \cap(s t)^{-}\right) \cup\left((S \backslash P) \cap(s t)^{+}\right)$such that $(P \backslash\{r\},\{r, s, t\})$ is a convex $k$-pair of $S$ compatible with $\mathcal{F}$.

If $r \in(S \backslash P) \cap(s t)^{+}$, then $\tau=\mathcal{Q}_{k}(P \backslash\{r\},\{r, s, t\})=\mathcal{Q}_{k}(P,\{r, s, t\})$ is the type-1 triangle $g(P \cup\{r\}) g(P \cup\{s\}) g(P \cup\{t\})$.

If $r \in P \cap(s t)^{-}$, then $\tau=\mathcal{Q}_{k}(P \backslash\{r\},\{r, s, t\})$ is the type-2 triangle $g(P \cup\{s\}) g(P \cup$ $\{t\}) g((P \backslash\{r\}) \cup\{s, t\})$.

In both cases, $e=[g(P \cup\{s\}), g(P \cup\{t\})]$ is an edge of $\tau$.

(ii) If $e$ is not an edge of $\mathcal{Q}_{k}(S),(s t)^{+} \cap S \neq P$ and $(s t)^{-} \cap S \neq P$. As in (i), from Theorem 3.7, there exists a point $r \in\left(P \cap(s t)^{-}\right) \cup\left((S \backslash P) \cap(s t)^{+}\right)$such that $(P \backslash\{r\},\{r, s, t\})$ is a convex $k$-pair of $S$ compatible with $\mathcal{F}$ and $e$ is an edge of the triangle $\tau=\mathcal{Q}_{k}(P \backslash\{r\},\{r, s, t\})$. Exchanging $s$ and $t$, from Theorem 3.7, there also exists a point $r^{\prime} \in\left(P \cap(s t)^{+}\right) \cup\left((S \backslash P) \cap(s t)^{-}\right)$ such that $\left(P \backslash\left\{r^{\prime}\right\},\left\{r^{\prime}, s, t\right\}\right)$ is a convex $k$-pair of $S$ compatible with $\mathcal{F}$ and $e$ is an edge of the triangle $\tau^{\prime}=\mathcal{Q}_{k}\left(P \backslash\left\{r^{\prime}\right\},\left\{r^{\prime}, s, t\right\}\right)$. Since $r \neq r^{\prime}$, one has $\tau \neq \tau^{\prime}$. Since $(P \backslash\{r\},\{r, s, t\})$ and $\left(P \backslash\left\{r^{\prime}\right\},\left\{r^{\prime}, s, t\right\}\right)$ are compatible with $\mathcal{F}$, they are compatible with each other, from Proposition 2.11 (ii). Thus, from Proposition 3.4, $\tau$ and $\tau^{\prime}$ are on both sides of $e$.

All the results of this section lead to the next theorem.

Theorem 3.9. For every maximal family $\mathcal{F}$ of subsets of $S$ separable by convex pseudo-circles, the $k$-set polygons of the convex $k$-pairs of $S$ compatible with $\mathcal{F}$ form the set of vertices, edges, and triangles of a triangulation of $\mathcal{Q}_{k}(S)$.

Proof. Let $\varphi$ be the set of $k$-set polygons of the convex $k$-pairs of $S$ compatible with $\mathcal{F}$. The set $\varphi$ contains vertices, edges, and triangles. From Proposition 3.3, the endpoints of the edges of $\varphi$ and the vertices and edges of the triangles of $\varphi$ are in $\varphi$. From Propositions 2.11 (ii) and 3.4 , two elements of $\varphi$ can only intersect at their vertices, or along their edges in the case of triangles.

Suppose the elements of $\varphi$ does not cover $\mathcal{Q}_{k}(S)$ and let $x \in \mathcal{Q}_{k}(S) \backslash \varphi$ and $y \in \mathbb{R}^{2} \backslash \mathcal{Q}_{k}(S)$. Since $\varphi$ has finitely many elements, $y$ can always be chosen in such a way that the segment $[x, y]$ meets no vertex in $\varphi$. From Propositions 3.5 and 3.6, every edge of $\mathcal{Q}_{k}(S)$ is in $\varphi$ and, therefore, $[x, y]$ cuts at least one edge of $\varphi$. Since the edges of the triangles of $\varphi$ are edges of $\varphi$, the element of $\varphi$ closest to $x$ cut by $[x, y]$ is an edge $c$ of $\varphi$. The segments $[x, y]$ and $c$ cut properly since the endpoints of $c$ are vertices of $\varphi$, and thus cannot belong to $[x, y]$. It follows that $c$ is either an edge of the boundary of $\mathcal{Q}_{k}(S)$ adjacent to no triangle of $\varphi$, or an edge in the interior of $\mathcal{Q}_{k}(S)$ adjacent to at most one triangle of $\varphi$. This contradicts Corollary 3.8, hence $\varphi$ covers $\mathcal{Q}_{k}(S)$.

This triangulation is called the order-k centroid triangulation of $S$ associated with $\mathcal{F}$; See Figure 6.

\section{Enumeration}

The aim of this section is to count the number of elements of a maximal family $\mathcal{F}$ of subsets of $S$ separable by convex pseudo-circles. For all $k \in\{1, \ldots, n\}$, let $\mathcal{T}_{k}$ be the order- $k$ centroid triangulation of $S$ associated with $\mathcal{F}$. From Theorem 3.9, the number $\left|\mathcal{F}_{k}\right|$ of elements in $\mathcal{F}$ of size $k$ equals the number of vertices of $\mathcal{T}_{k}$.

Remark 4.1. (i) Note that $\mathcal{T}_{1}$ is just a triangulation of $S$. Indeed, it is easy to check that every singleton of $S$ belongs to $\mathcal{F}$.

(ii) Since $\mathcal{F}_{n}=\{S\}, \mathcal{T}_{n}$ is reduced to a vertex. 


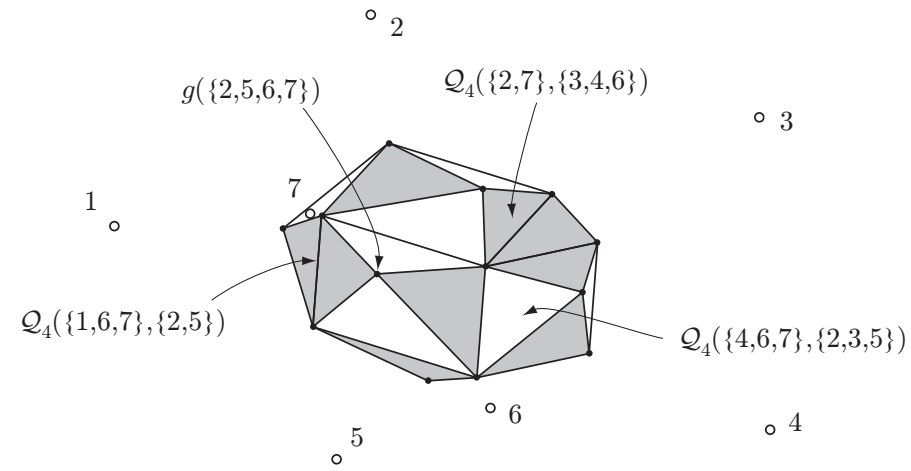

Figure 6: An order-4 centroid triangulation of 7 points, with type- 1 triangles in white and type-2 triangles in grey.

From Remark 4.1, $\left|\mathcal{F}_{1}\right|=n$ and $\left|\mathcal{F}_{n}\right|=1$. Let now $k \in\{2, \ldots, n-1\}$. To find the number of vertices of $\mathcal{T}_{k}$, we have at our disposal the two usual enumeration formulas that give the number of vertices in terms of the numbers of edges and of triangles in a triangulation. In order to find a third formula for these numbers, we study relationships between the triangulations $\mathcal{T}_{k}$ and $\mathcal{T}_{k-1}$.

Lemma 4.2. (i) For every convex pair $(P, Q)$ of $S$ compatible with $\mathcal{F}$ such that $|Q|=3$ and $|P|=k-2, \mathcal{Q}_{k}(P, Q)$ is a triangle of type 2 of $\mathcal{T}_{k}$ and $\mathcal{Q}_{k-1}(P, Q)$ is a triangle of type 1 of $\mathcal{T}_{k-1}$. $\mathcal{T}_{k-1}$.

(ii) The number of triangles of type 2 of $\mathcal{T}_{k}$ is equal to the number of triangles of type 1 of

Proof. (i) From the definition of convex $k$-pairs, every convex pair $(P, Q)$ of $S$ such that $|Q|=3$ and $|P|=k-2$ is both a convex $k$-pair and a convex $(k-1)$-pair of $S$. From Theorem 3.9, if $(P, Q)$ is compatible with $\mathcal{F}, \mathcal{Q}_{k}(P, Q)$ is a triangle of $\mathcal{T}_{k}$ and $\mathcal{Q}_{k-1}(P, Q)$ is a triangle of $\mathcal{T}_{k-1}$. These two triangles are respectively of type 2 and of type 1 .

(ii) Since every type- 2 triangle of $\mathcal{T}_{k}$ is the $k$-set polygon of a convex pair $(P, Q)$ compatible with $\mathcal{F}$ such that $|Q|=3$ and $|P|=k-2$, and since every type- 1 triangle of $\mathcal{T}_{k-1}$ is the $(k-1)$-set polygon of such a convex pair, it follows from (i) that the number of type-2 triangles of $\mathcal{T}_{k}$ equals the number of type- 1 triangles of $\mathcal{T}_{k-1}$.

For a $k$-subset $T$ of $S$, if the union of the edges and triangles of $\mathcal{T}_{k-1}$ of the form $\mathcal{Q}_{k-1}(P, Q)$ with $P \cup Q=T$ is nonempty, then this union is called the domain of $T$ in $\mathcal{T}_{k-1}$.

Remark 4.3. (i) From Observation 3.2, every edge and every type-2 triangle belongs to one and only one domain. The edges of a type- 2 triangle belong to the same domain as the triangle. The type-1 triangles belong to no domain.

(ii) All triangles of $\mathcal{T}_{1}$ are of type 1 and all edges of $\mathcal{T}_{1}$ belong to distinct domains. Hence, the domains of $\mathcal{T}_{1}$ are the edges of $\mathcal{T}_{1}$.

Lemma 4.4. (i) For every $k$-subset $T$ of $S, T$ admits a domain in $\mathcal{T}_{k-1}$ if and only if $g(T)$ is a vertex of $\mathcal{T}_{k}$.

(ii) The number of domains in $\mathcal{T}_{k-1}$ is equal to the number of vertices of $\mathcal{T}_{k}$.

Proof. (i) If the $k$-subset $T$ of $S$ admits a domain in $\mathcal{T}_{k-1}$ then, from Remark 4.3, this domain contains at least one edge and this edge is of the form $\mathcal{Q}_{k-1}(P,\{s, t\})$, with $P \cup\{s, t\}=T$. Thus, $T$ is a subset of $(P,\{s, t\})$ and, from Proposition 2.11 (i), it is in $\mathcal{F}$. Therefore, $g(T)$ is a vertex of $\mathcal{T}_{k}$.

Conversely, suppose the centroid $g(T)$ of the $k$-subset $T$ of $S$ is a vertex of $\mathcal{T}_{k}$. Let $g\left(T_{0}\right), \ldots, g\left(T_{m}\right)$ be the neighbors of $g(T)$ in $\mathcal{T}_{k}$ in counterclockwise order around $g(T)$, and such that: 
- if $g(T)$ is not a vertex of $\mathcal{Q}_{k}(S)$, then $g\left(T_{0}\right)=g\left(T_{m}\right)$,

- if $g(T)$ is a vertex of $\mathcal{Q}_{k}(S)$, then $g\left(T_{0}\right)$ and $g\left(T_{m}\right)$ are respectively the successor and the predecessor of $g(T)$ on $\partial \mathcal{Q}_{k}(S)$.

The points $g\left(T_{0}\right), \ldots, g\left(T_{m}\right)$ are the vertices of a star-shaped polygonal chain with respect to $g(T)$. The chain is closed if $g(T)$ is not a vertex of $\mathcal{Q}_{k}(S)$ and is open otherwise. Since, for every $i \in\{0, \ldots, m\},\left[g(T), g\left(T_{i}\right)\right]$ is an edge of $\mathcal{T}_{k}$, there exist, from Observation $3.2, t_{i} \in T$ and $s_{i} \in S \backslash T$ such that $T_{i}=\left(T \backslash\left\{t_{i}\right\}\right) \cup\left\{s_{i}\right\}$.

We first show that the $t_{i}$ cannot all be equal. If all $t_{i}$ are equal to a same point $t$, we have $T_{i}=(T \backslash\{t\}) \cup\left\{s_{i}\right\}$, for every $i \in\{0, \ldots, m\}$. Since $k \geq 2, T \backslash\{t\}$ contains at least on point and, by a homothety with center $g(T \backslash\{t\}), s_{0}, \ldots, s_{m}$ are the vertices of a star-shaped polygonal chain with respect to $t$. Furthermore, from Proposition 3.5, if $g(T)$ is a vertex of $\mathcal{Q}_{k}(S), T \backslash\{t\} \subset\left(s_{m} t\right)^{-} \cap\left(s_{0} t\right)^{+}$and $s_{i} \in\left(s_{m} t\right)^{+} \cap\left(s_{0} t\right)^{-}$, for every $i \in\{1, \ldots, m-1\}$. Hence, for every point $p$ of $T \backslash\{t\}$, there exists in all cases an index $i$ of $\{0, \ldots, m-1\}$ such that $p \in\left(s_{i} t\right)^{+} \cap\left(s_{i+1} t\right)^{-}$. Therefore, $t \in \operatorname{conv}\left(\left\{p, s_{i}, s_{i+1}\right\}\right)$. Now, $g(T), g\left(T_{i}\right)$, and $g\left(T_{i+1}\right)$ are the three vertices of a triangle $\mathcal{Q}_{k}\left(T \backslash\{t\},\left\{t, s_{i}, s_{i+1}\right\}\right)$ of $\mathcal{T}_{k}$. Thus, by the definition of a convex pair, $t$ is an extreme point of $T \cup\left\{s_{i}, s_{i+1}\right\}$, in contradiction with $t \in \operatorname{conv}\left(\left\{p, s_{i}, s_{i+1}\right\}\right)$. It follows that the $t_{i}, i \in\{0, \ldots, m\}$, are not all equal.

Hence, there exists $i \in\{0, \ldots, m-1\}$ such that $t_{i} \neq t_{i+1}$. The triangle of $\mathcal{T}_{k}$ with vertices $g(T), g\left(T_{i}\right)$, and $g\left(T_{i+1}\right)$ is then the $k$-set polygon of a convex $k$-pair $\left(T \backslash\left\{t_{i}, t_{i+1}\right\},\left\{t_{i}, t_{i+1}, s_{i}\right\}\right)$ and is a type-2 triangle (from Observation 3.2 we necessarily have $s_{i+1}=s_{i}$ ). From Lemma 4.2 (i), $\mathcal{Q}_{k-1}\left(T \backslash\left\{t_{i}, t_{i+1}\right\},\left\{t_{i}, t_{i+1}, s_{i}\right\}\right)$ is a type- 1 triangle of the triangulation $\mathcal{T}_{k-1}$, and $\mathcal{Q}_{k-1}\left(T \backslash\left\{t_{i}, t_{i+1}\right\},\left\{t_{i}, t_{i+1}\right\}\right)$ is one of its edges. This edge belongs to the domain of $T$.

Thus, if $g(T)$ is a vertex of $\mathcal{T}_{k}, T$ admits a domain in $\mathcal{T}_{k-1}$.

(ii) For two convex $k$-subsets $T$ and $T^{\prime}$ of $\mathcal{F}, g(T)$ and $g\left(T^{\prime}\right)$ are two distinct vertices of $\mathcal{T}_{k}$, from Proposition 3.4, and the domains of $T$ and of $T^{\prime}$ in $\mathcal{T}_{k-1}$ are disjoint, from Remark 4.3. Thus, from (i), the number of vertices of $\mathcal{T}_{k}$ is equal to the number of domains in $\mathcal{T}_{k-1}$.

The next property on the shape of the domains will allow, together with Remark 4.3 (i) and Lemmas 4.4 (ii) and 4.2 (ii), to establish an enumeration formula for the vertices, edges, and triangles of centroid triangulations of consecutive orders.

Proposition 4.5. (i) The vertices of $\mathcal{T}_{k-1}$ that belong to a same domain are the extreme points of this domain.

(ii) The domains are convex.

Proof. (i) Let $T$ be a convex $k$-subset of $\mathcal{F}$. From Remark 4.3, the vertices of the domain of $T$ in $\mathcal{T}_{k-1}$ are the endpoints of the edges of $\mathcal{T}_{k-1}$ of the form $\mathcal{Q}_{k-1}(T \backslash\{s, t\},\{s, t\})$, where $s$ and $t$ are two extreme points of $T$. Thus, every vertex of the domain of $T$ is of the form $g(T \backslash\{s\})$, where $s$ is an extreme point of $T$. By a homothety with center $g(T), g(T \backslash\{s\})$ is an extreme point of the set of vertices of the domain of $T$.

(ii) If the domain of $T$ contains only two vertices, it is reduced to a line segment and is convex.

Now we show that, if the domain of $T$ in $\mathcal{T}_{k-1}$ contains at least three vertices, then every point in the convex hull of these vertices belongs to the domain of $T$.

(ii.1) Every point $x$ in this convex hull belongs to a triangle whose three vertices are vertices of the domain of $T$. From the proof of (i), these three vertices are of the form $g(T \backslash\{r\})$, $g(T \backslash\{s\})$, and $g(T \backslash\{t\})$, where $r, s$, and $t$ are extreme points of $T$. Since $\operatorname{conv}(T) \cap S=T$, $(T \backslash\{r, s, t\},\{r, s, t\})$ is a convex $(k-1)$-pair and its $(k-1)$-set polygon is the triangle $g(T \backslash\{r\})$ $g(T \backslash\{s\}) g(T \backslash\{t\})$, which contains the point $x$.

If $(T \backslash\{r, s, t\},\{r, s, t\})$ is compatible with $\mathcal{F}$, its $(k-1)$-set polygon is a triangle of $\mathcal{T}_{k-1}$ that belongs to the domain of $T$, and $x$ also belongs to this domain. 
Otherwise, $x$ belongs to a triangle of $\mathcal{T}_{k-1}$, which is the $(k-1)$-set polygon of a convex $(k-1)$-pair $(P, Q)$ of $S$ compatible with $\mathcal{F}$. From Proposition 3.4, $(P, Q)$ is then incompatible with $(T \backslash\{r, s, t\},\{r, s, t\})$. Therefore, from Lemma 2.10, some subsets of $(P, Q)$ are incompatible with some subsets of $(T \backslash\{r, s, t\},\{r, s, t\})$, and are also incompatible with $(T \backslash\{r, s, t\},\{r, s, t\})$, from the same lemma. Let $T^{\prime}$ be any one of the subsets of $(P, Q)$ incompatible with $(T \backslash$ $\{r, s, t\},\{r, s, t\})$. From Proposition 2.11 (i), $T^{\prime}$ is in $\mathcal{F}$. Since $T \backslash\{r\}, T \backslash\{s\}, T \backslash\{t\}$, and $T$ are also in $\mathcal{F}$, these four convex subsets are compatible with $T^{\prime}$, and one has the five following relations:

$$
\begin{aligned}
& \operatorname{conv}\left(T^{\prime} \backslash(T \backslash\{r, s, t\})\right) \cap \operatorname{conv}\left(T \backslash T^{\prime}\right) \neq \emptyset \\
& \operatorname{conv}\left(T^{\prime} \backslash(T \backslash\{r\})\right) \cap \operatorname{conv}\left((T \backslash\{r\}) \backslash T^{\prime}\right)=\emptyset \\
& \operatorname{conv}\left(T^{\prime} \backslash(T \backslash\{s\})\right) \cap \operatorname{conv}\left((T \backslash\{s\}) \backslash T^{\prime}\right)=\emptyset \\
& \operatorname{conv}\left(T^{\prime} \backslash(T \backslash\{t\})\right) \cap \operatorname{conv}\left((T \backslash\{t\}) \backslash T^{\prime}\right)=\emptyset \\
& \operatorname{conv}\left(T^{\prime} \backslash T\right) \cap \operatorname{conv}\left(T \backslash T^{\prime}\right)=\emptyset
\end{aligned}
$$

Setting $W=\{r, s, t\} \cap T^{\prime}$, relation (1) can also be written

$$
\operatorname{conv}\left(T^{\prime} \backslash(T \backslash W)\right) \cap \operatorname{conv}\left((T \backslash W) \backslash T^{\prime}\right) \neq \emptyset .
$$

It then follows from the relations (2), (3), (4), and (5) that $|W| \geq 2$, and therefore one of the four following assertions holds:

$$
\begin{aligned}
& W=\{r, s\} \text { and } \operatorname{conv}\left(T^{\prime} \backslash(T \backslash\{r, s, t\})\right)=\operatorname{conv}\left(\left(T^{\prime} \backslash T\right) \cup\{r, s\}\right) \\
& W=\{r, t\} \text { and } \operatorname{conv}\left(T^{\prime} \backslash(T \backslash\{r, s, t\})\right)=\operatorname{conv}\left(\left(T^{\prime} \backslash T\right) \cup\{r, t\}\right) \\
& W=\{s, t\} \text { and } \operatorname{conv}\left(T^{\prime} \backslash(T \backslash\{r, s, t\})\right)=\operatorname{conv}\left(\left(T^{\prime} \backslash T\right) \cup\{s, t\}\right) \\
& W=\{r, s, t\} \text { and } \operatorname{conv}\left(T^{\prime} \backslash(T \backslash\{r, s, t\})\right)=\operatorname{conv}\left(\left(T^{\prime} \backslash T\right) \cup\{r, s, t\}\right)
\end{aligned}
$$

(ii.2) Suppose $T^{\prime} \backslash T \neq \emptyset$. In this case, $\operatorname{conv}\left(\left(T^{\prime} \backslash T\right) \cup\{r, s, t\}\right)$ is the union $\operatorname{conv}\left(\left(T^{\prime} \backslash T\right) \cup\right.$ $\{r, s\}) \cup \operatorname{conv}\left(\left(T^{\prime} \backslash T\right) \cup\{r, t\}\right) \cup \operatorname{conv}\left(\left(T^{\prime} \backslash T\right) \cup\{s, t\}\right)$. Indeed, $\operatorname{conv}\left(\left(T^{\prime} \backslash T\right) \cup\{r, s, t\}\right)$ can be covered by triangles that all have a same point of $T^{\prime} \backslash T$ as vertex, and which are therefore distinct from triangle rst.

It then follows from (1) and from (6), (7), (8), (9) that, within a permutation of $r, s, t$,

$$
\operatorname{conv}\left(\left(T^{\prime} \backslash T\right) \cup\{r, s\}\right) \cap \operatorname{conv}\left(T \backslash T^{\prime}\right) \neq \emptyset \text { and }\{r, s\} \subseteq T^{\prime} \cap T .
$$

The convex hull conv $\left(\left(T^{\prime} \backslash T\right) \cup\{r, s\}\right)$ has at least three vertices and does not contain any point of $T \backslash T^{\prime}$ since $\operatorname{conv}\left(T^{\prime}\right) \cap S=T^{\prime}$. In the same way, no point of $T^{\prime} \backslash T$ belongs to $\operatorname{conv}\left(T \backslash T^{\prime}\right)$ since $\operatorname{conv}(T) \cap S=T$. Since no three points in $S$ are collinear, it follows that $T \backslash T^{\prime}$ contains two points $p$ and $p^{\prime}$ such that the line segment $\left[p, p^{\prime}\right]$ cuts properly two edges of $\operatorname{conv}\left(\left(T^{\prime} \backslash T\right) \cup\{r, s\}\right)$. Now, all edges of $\operatorname{conv}\left(\left(T^{\prime} \backslash T\right) \cup\{r, s\}\right)$ are edges of $\operatorname{conv}\left(\left(T^{\prime} \backslash T\right) \cup\{r\}\right)$, except the two edges out of $s$, when $s$ is a vertex of $\operatorname{conv}\left(\left(T^{\prime} \backslash T\right) \cup\{r, s\}\right)$. In the same way, all edges of $\operatorname{conv}\left(\left(T^{\prime} \backslash T\right) \cup\{r, s\}\right)$ are edges of $\operatorname{conv}\left(\left(T^{\prime} \backslash T\right) \cup\{s\}\right)$, except possibly the two edges out of $r$. It follows that the edges of $\operatorname{conv}\left(\left(T^{\prime} \backslash T\right) \cup\{r, s\}\right)$ belong to the set formed by the edges of $\operatorname{conv}\left(\left(T^{\prime} \backslash T\right) \cup\{r\}\right)$, the edges of $\operatorname{conv}\left(\left(T^{\prime} \backslash T\right) \cup\{s\}\right)$, and the segment $r s$. But $\operatorname{conv}\left(T \backslash T^{\prime}\right)$ cannot cut two edges of this set since, with $\{r, s\} \subseteq T^{\prime}$, (2) and (3) become $\operatorname{conv}\left(\left(T^{\prime} \backslash T\right) \cup\{r\}\right) \cap \operatorname{conv}\left(T \backslash T^{\prime}\right)=\emptyset$ and $\operatorname{conv}\left(\left(T^{\prime} \backslash T\right) \cup\{s\}\right) \cap \operatorname{conv}\left(T \backslash T^{\prime}\right)=\emptyset$.

Thus, the hypothesis $T^{\prime} \backslash T \neq \emptyset$ is absurd and therefore $T^{\prime} \subseteq T$. Furthermore, since $\operatorname{conv}\left(T^{\prime}\right) \cap S=T^{\prime}$, we have $\operatorname{conv}\left(T^{\prime} \backslash(T \backslash\{r, s, t\})\right) \cap\left(T \backslash T^{\prime}\right)=\emptyset$, and it follows from (1) that $\left|T \backslash T^{\prime}\right| \geq 2$.

Finally, for every subset $T^{\prime}$ of $(P, Q)$ incompatible with the convex pair $(T \backslash\{r, s, t\},\{r, s, t\})$, we have $T^{\prime} \subsetneq T$ and $\left|T^{\prime}\right|<k-1$. 
(ii.3) For every subset $T^{\prime}$ of $(P, Q)$ incompatible with $(T \backslash\{r, s, t\},\{r, s, t\})$, the set $T^{\prime} \cup(Q \backslash T)$ is also a subset of $(P, Q)$, and is convex from Lemma 2.8. Furthermore, relation (1) implies

$$
\operatorname{conv}\left(\left(T^{\prime} \cup(Q \backslash T)\right) \backslash(T \backslash\{r, s, t\})\right) \cap \operatorname{conv}\left(T \backslash\left(T^{\prime} \cup(Q \backslash T)\right)\right) \neq \emptyset
$$

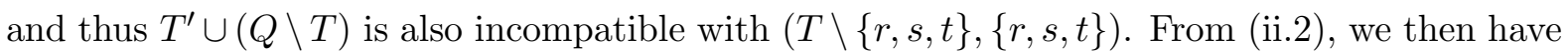
$T^{\prime} \cup(Q \backslash T) \subsetneq T$, i.e., $Q \subseteq T$. Since $T^{\prime}$ is a subset of $(P, Q)$, one also has $P \subseteq T^{\prime} \subsetneq T$. Hence $P \cup Q \subseteq T$. Furthermore, from (ii.2), $\left|T^{\prime}\right|<k-1$, which implies $|P|<k-1$ and, from the definition of a convex $(k-1)$-pair, $|P \cup Q|>k-1$. Since $|T|=k$, it follows that $P \cup Q=T$ and thus that $\mathcal{Q}_{k-1}(P, Q)$ belongs to the domain of $T$.

Hence, every point $x$ in the convex hull of the vertices of the domain of $T$ belongs to the domain of $T$. This proves that this domain is convex.

This proposition, which shows that the domains of an order- $k$ centroid triangulation are triangulations of convex polygons, can be compared to a result of Lee [9], according to which the order- $k$ Voronoi diagram is composed of subgraphs that are binary trees. Up to the duality, we thus obtain the same enumeration formula as Lee, implying that the number of vertices of $\mathcal{T}_{k}$ equals the number of regions of the order- $k$ Voronoi diagram. Denoting, for all $i \in\{0, \ldots, n\}$, by $a_{i}(S)$ the number of $i$-sets of $S$, we then get the following results.

Proposition 4.6. Let $k \in\{1, \ldots, n\}$.

(i) The number of vertices of $\mathcal{T}_{k}$ is

$$
\left|\mathcal{F}_{k}\right|=2 k n-n-k^{2}+1-\sum_{i=1}^{k-1} a_{i}(S) .
$$

(ii) We have

$$
\left|\mathcal{F}_{k}\right|+\left|\mathcal{F}_{n-k+1}\right|=2 k n-2 k^{2}+2 k-n+1 .
$$

In particular, if $n$ is odd and $m=(n+1) / 2$ then $\left|\mathcal{F}_{m}\right|=m^{2}-m+1$.

(iii) $\mathcal{F}_{k}$ is a maximal family of compatible convex $k$-subsets of $S$.

Proof. (i) When $k=n$, the formula is verified since $\left|\mathcal{F}_{n}\right|=1$ and since $\sum_{i=1}^{n-1} a_{i}(S)=n(n-1)$. This well known result can be obtained for example from Proposition 3.5, which states that the number $a_{i}(S)$ of $i$-sets of $S$ equals the number of oriented straight lines passing through any two points of $S$ and having precisely $i-1$ points on their right side. Therefore, $\sum_{i=1}^{n-1} a_{i}(S)$ is equal to the total number of ordered pairs of points of $S$.

For all $k \in\{1, \ldots, n-1\}$, let $v_{k}, e_{k}, t_{1, k}$, and $t_{2, k}$ be the respective numbers of vertices, edges, type- 1 triangles, and type- 2 triangles of $\mathcal{T}_{k}$. The usual enumeration formulas for triangulations give

$$
e_{k}=3 v_{k}-a_{k}(S)-3 \text { and } t_{1, k}+t_{2, k}=2 v_{k}-a_{k}(S)-2,
$$

where $a_{k}(S)$ is the number of vertices of $\mathcal{Q}_{k}(S)$, i.e., the number of $k$-sets of $S$, from Proposition 3.5. For every $k$-subset $T$ of $S, k \in\{2, \ldots, n-1\}$, that admits a domain in $\mathcal{T}_{k-1}$, we denote respectively $e_{T, k-1}$ and $t_{T, k-1}$ the numbers of edges and of triangles of the domain of $T$. From Proposition 4.5, these edges and triangles form a triangulation of a convex polygon and they therefore satisfy $e_{T, k-1}=2 t_{T, k-1}+1$. From Remark 4.3, every edge and every type-2 triangle of $\mathcal{T}_{k-1}$ belongs to one and only one domain. From Lemma 4.4, the number of domains of $\mathcal{T}_{k-1}$ is equal to the number of vertices of $\mathcal{T}_{k}$. Summing the preceding relation over all domains of $\mathcal{T}_{k-1}$, one then gets $e_{k-1}=2 t_{2, k-1}+v_{k}$. Lemma 4.2 (ii) then implies, for all $k \in\{3, \ldots, n-1\}$, $e_{k-1}+e_{k-2}=2\left(t_{1, k-2}+t_{2, k-2}\right)+v_{k}+v_{k-1}$. Using (10), one obtains

$$
v_{k}-2 v_{k-1}+v_{k-2}=a_{k-2}(S)-a_{k-1}(S)-2 .
$$


To solve this induction equation, write it in the form

$$
(k-i+1)\left(v_{i}-2 v_{i-1}+v_{i-2}\right)=(k-i+1)\left(a_{i-2}(S)-a_{i-1}(S)-2\right),
$$

for all $i \in\{3, \ldots, k\}$, and sum member by member over all these $i$. One then gets

$$
v_{k}-(k-1) v_{2}+(k-2) v_{1}=(k-2) a_{1}(S)-(k-2)(k-1)-\sum_{i=2}^{k-1} a_{i}(S) .
$$

From Remark 4.1, $v_{1}=\left|\mathcal{F}_{1}\right|=n$. From Remark 4.3, the domains of $\mathcal{T}_{1}$ are the edges of $\mathcal{T}_{1}$ and thus, from Lemma 4.4 (ii) and from the relations (10), $v_{2}=3 v_{1}-a_{1}(S)-3$. Substituting in the preceding equality, we obtain

$$
v_{k}=2 k n-n-k^{2}+1-\sum_{i=1}^{k-1} a_{i}(S)
$$

for all $k \in\{3, \ldots, n-1\}$. The equality holds true for $k=2$, and also for $k=1$ by setting $\sum_{1}^{0}=0$.

(ii) Since the number of $i$-sets of $S$ equals the number of $(n-i)$-sets of $S$, it follows from (i), for all $k \in\{1, \ldots, n\}$,

$$
\left|\mathcal{F}_{n-k+1}\right|=n^{2}-k^{2}-n+2 k-\sum_{i=k}^{n-1} a_{i}(S)
$$

Therefore,

$$
\left|\mathcal{F}_{k}\right|+\left|\mathcal{F}_{n-k+1}\right|=n^{2}+2 k n-2 n-2 k^{2}+2 k+1-\sum_{i=1}^{n-1} a_{i}(S)=2 k n-2 k^{2}+2 k-n+1 .
$$

If $n$ is odd, setting $m=(n+1) / 2$, we then get $2\left|F_{m}\right|=2 m^{2}-2 m+2$.

(iii) The result is obviously true for $\mathcal{F}_{n}=\{S\}$. For $k<n$, let $\mathcal{F}_{k}^{\prime}$ be a maximal family of compatible convex $k$-subsets of $S$ containing $\mathcal{F}_{k}$. Complete $\mathcal{F}_{k}^{\prime}$ to a maximal family $\mathcal{F}^{\prime}$ of compatible convex subsets of $S$. From (i), one has $\left|\mathcal{F}_{k}^{\prime}\right|=2 k n-n-k^{2}+1-\sum_{i=1}^{k-1} a_{i}(S)=\left|\mathcal{F}_{k}\right|$. Therefore, $\mathcal{F}_{k}=\mathcal{F}_{k}^{\prime}$, and $\mathcal{F}_{k}$ is a maximal family of compatible convex $k$-subsets of $S$.

Recall the results stated in the introduction.

Theorem 1.1 Let $S$ be a set of $n$ points in the plane in general position. Every inclusionmaximal family $\mathcal{F}$ of subsets of $S$ separable by convex pseudo-circles has c $(3, n)=\left(\begin{array}{l}n \\ 0\end{array}\right)+\left(\begin{array}{l}n \\ 1\end{array}\right)+$ $\left(\begin{array}{l}n \\ 2\end{array}\right)+\left(\begin{array}{l}n \\ 3\end{array}\right)$ elements.

Theorem 1.2 Let $S$ be a set of $n$ points in the plane in general position and $k \in\{1, \ldots, n\}$. Every inclusion-maximal family of $k$-subsets of $S$ separable by convex pseudo-circles admits $2 k n-n-k^{2}+1-\sum_{i=1}^{k-1} a_{i}(S)$ elements.

Theorem 1.2 is an immediate consequence of Proposition 4.6 (i) and (iii). It shows especially that the number of elements in a maximal family of $k$-subsets of $S$ separable by convex pseudocircles is an invariant of $S$ and $k$. Since this number is also equal to the number of $k$-subsets of $S$ separable by circles when no four points are cocircular [9], a maximal family of $k$-subsets of $S$ separable by circles is also maximal for convex pseudo-circles when $S$ is in general position.

In the same way, Proposition 4.6 (ii) and (iii) shows that the sum of the numbers of elements in two maximal families of $k$-subsets and of $(n-k+1)$-subsets of $S$, each one separable by 
convex pseudo-circles, is an invariant of $n$ and $k$; It depends neither on the choice of the two maximal families, nor on the distribution of the points of $S$ (as long as no three of them are collinear). As a consequence, if $n$ is odd, the number of $((n+1) / 2)$-subsets separable by convex pseudo-circles is an invariant of $n$.

Proof of Theorem 1.1. Since $\mathcal{F}=\left(\bigcup_{k=1}^{n} \mathcal{F}_{k}\right) \cup\{\emptyset\}$ and since these subsets are disjoint, one has

$$
|\mathcal{F}|=\sum_{k=1}^{n}\left|\mathcal{F}_{k}\right|+1=\frac{1}{2} \sum_{k=1}^{n}\left(\left|\mathcal{F}_{k}\right|+\left|\mathcal{F}_{n-k+1}\right|\right)+1 .
$$

Hence, from Proposition 4.6 (ii),

$$
|\mathcal{F}|=\frac{n^{3}+5 n+6}{6}=\left(\begin{array}{l}
n \\
0
\end{array}\right)+\left(\begin{array}{l}
n \\
1
\end{array}\right)+\left(\begin{array}{l}
n \\
2
\end{array}\right)+\left(\begin{array}{l}
n \\
3
\end{array}\right) .
$$

\section{Proof of Theorem 3.7}

\subsection{Comments on the proof}

In case one only deals with separating curves that are (true) circles, the proof of Theorem 3.7 is very easy. Indeed, in this case $(P,\{s, t\})$ admits a separating circle $\gamma$ which passes through $s, t$ and encloses exactly $P$. The circle $\gamma$ can be continuously deformed (remaining in the family of circles passing through $s, t)$ until it meets a third point $r$ of $S$. If $(s t)^{+} \cap S \neq P$ and if we perform the deformation only towards $(s t)^{+}$, we find exactly one point $r$ (assuming no four cocircular points in $S$ ): $r$ is either a point of $S \backslash P$ in $(s t)^{+}$or a point of $P$ in $(s t)^{-}$. Then, the new circle $\gamma^{\prime}$ encloses exactly $P \backslash\{r\}$ and the pair $(P \backslash\{r\},\{r, s, t\})$ is convex. Furthermore, $(P \backslash\{r\},\{r, s, t\})$ is compatible with all convex pairs that admit circles as separating curves, since circles are always compatible.

In the general case, finding a convex pair $(P \backslash\{r\},\{r, s, t\})$ is even easier; Usually there are several such pairs. The difficulty is to prove that there exists at least one that is compatible with all elements of $\mathcal{F}$. In particular, an analogous method by deformation is unclear for pseudo-circles. Actually, if one tries to deform a pseudo-circle belonging to a given family of pseudo-circles, it is far from evident to keep the compatibility with the family. It can happen that, while deforming one curve, some others have to be modified at the same time, as shows the following example: For every point $q_{i}, i \in\{1,2,3\}$ of Figure $7, \gamma$ can be deformed into a convex curve $\gamma_{i}$ passing through $\left\{q_{i}, s, t\right\}$ and enclosing exactly $\left\{p_{1}, p_{2}\right\}$. Now, the curve $\gamma_{1}$ will be incompatible with $\Gamma_{1}$ and the curve $\gamma_{3}$ will be incompatible with $\Gamma_{3}$. However these incompatibilities are not of the same nature: It is possible to reduce $\Gamma_{3}$ in order to ensure its compatibility with $\gamma_{3}$, while this is impossible for $\Gamma_{1}$ and $\gamma_{1}$. Indeed, since the segments $\left[t, q_{2}\right]$ and $\left[p_{1}, q_{1}\right]$ cross, any curve enclosing $t$ and $q_{2}$ will be incompatible with any curve through $q_{1}$ and enclosing $p_{1}$. In the same way, $\gamma$ can be deformed into a convex curve passing through $\left\{p_{1}, s, t\right\}$ and enclosing $p_{2}$, or to a convex curve passing through $\left\{p_{2}, s, t\right\}$ and enclosing $p_{1}$, but the curve passing through $p_{2}$ will be incompatible with $\Gamma_{2}$.

Furthemore, it can be deduced from Propositions 2.11 and 3.4 that there is at most one such pair $(P \backslash\{r\},\{r, s, t\})$ compatible with $\mathcal{F}$, when $\mathcal{F}$ is maximal. This means that, among all the possible convex pairs $(P \backslash\{r\},\{r, s, t\})$, at most one could be reached by a continuous deformation keeping the compatibility, and for all others there are obstructions. A significant part of our proof consists in describing these obstructions: This is the role of the "lockers" described in Proposition 5.3.

Another strategy of proof could be to adapt the classical proof of the existence of the Delaunay triangulation using a lifting on a paraboloid in $\mathbb{R}^{3}$. Example 2 of Subsection 6.2 shows that this strategy fails. 


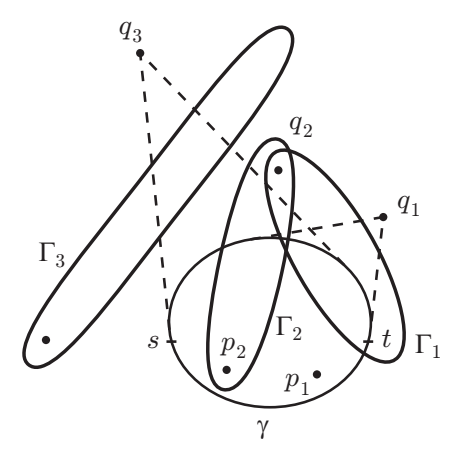

Figure 7: Obstructions for the deformation of a convex pseudo-circle.

We refer to the beginning of Section 2 above for the notation. We will use several times the following elementary property.

Remark 5.1. If $a, b, c, d$ are four distinct points in the plane such that $a \in(b c)^{-}$and $d \in$ $(a b)^{+} \cap(a c)^{-}$, then $a \in \operatorname{conv}(\{b, c, d\})$.

Proof. Since $d \in(a b)^{+}$, we have $a \in(d b)^{-}$; Similarly $d \in(a c)^{-}$implies $a \in(c d)^{-}$. Therefore we have $a \in(b c)^{-} \cap(c d)^{-} \cap(d b)^{-}=\operatorname{conv}(\{b, c, d\})$.

\subsection{Strategy of proof of Theorem 3.7}

The proof proceeds by contradiction. We assume that counterexamples exist and, among them, we choose one, denoted by $(S, \mathcal{F})$, with $|S|$ minimal. This means two things.

Firstly, the fact that $(S, \mathcal{F})$ is a counterexample means that there exists a convex pair $\left(P_{0},\{s, t\}\right)$ compatible with $\mathcal{F}$ such that $(s t)^{+} \cap S \neq P_{0}$ and every $r \in\left(P_{0} \cap(s t)^{-}\right) \cup\left(\left(S \backslash P_{0}\right) \cap\right.$ $\left.(s t)^{+}\right)$is locked in the following sense: The pair $\left(P_{0} \backslash\{r\},\{r, s, t\}\right)$ is either nonconvex in $S$ or it is convex but incompatible with some $P \in \mathcal{F}$. In the latter case, we say that $P$ locks $r$. We will use the notations $\widetilde{P_{0}}=P_{0} \cup\{s, t\}, \mathbf{L}=P_{0} \cap(s t)^{-}$, and $\mathbf{U}=\left(S \backslash P_{0}\right) \cap(s t)^{+}=\left(S \backslash \widetilde{P_{0}}\right) \cap(s t)^{+}$. We assume that $[s, t]$ is a horizontal segment, $s$ on the left and $t$ on the right, hence $\mathbf{L}$ belongs to the lower half plane delimited by $(s t)$ and $\mathbf{U}$ belongs to the upper half plane. Whenever possible, elements of $\mathbf{L}$ will be denoted by lowercase letters and elements of $\mathbf{U}$ by uppercase letters. The lockings are described in Subsection 5.3.

Secondly, the minimality of $|S|$ implies that, for all $y \in S \backslash\{s, t\}$, if $\mathbf{L} \cup \mathbf{U} \neq\{y\}$, there exists $x \in(\mathbf{L} \cup \mathbf{U}) \backslash\{y\}$ which is unlocked by $y$ in the following sense: The pair $\left(P_{0} \backslash\{x, y\},\{x, s, t\}\right)$ is convex in $S \backslash\{y\}$ and compatible with the family $\mathcal{F}_{y}=\{P \backslash\{y\} ; P \in \mathcal{F}\}$. The relation " $y$ unlocks $x$ " will be denoted by $y \rightarrow x$.

Proposition 5.8 in the sequel shows that, if $\mathbf{L} \cup \mathbf{U}$ had only one element $y$, then $\left(P_{0} \backslash\right.$ $\{y\},\{y, s, t\})$ would automatically be a convex pair compatible with $\mathcal{F}$. In other words, because we consider a hypothetical minimal counterexample, the set $\mathbf{L} \cup \mathbf{U}$ must contain at least two elements.

Let $D(y)=\{x \in \mathbf{L} \cup \mathbf{U} ; y \rightarrow x\}$. We thus have

$$
D(y) \neq \emptyset \text { for all } y \in S \backslash\{s, t\} .
$$

A point $y$ can unlock a point $x$ in two different ways. The first way is by convexity, if the pair $\left(P_{0} \backslash\{x\},\{x, s, t\}\right)$ is nonconvex in $S$, and $\left(P_{0} \backslash\{x, y\},\{x, s, t\}\right)$ is convex in $S \backslash\{y\}$ and compatible with $\mathcal{F}_{y}$. Unlockings by convexity are described in Subsection 5.5.

The second way is by compatibility, when $\left(P_{0} \backslash\{x\},\{x, s, t\}\right)$ is convex in $S$ but incompatible with some $P \in \mathcal{F}$, and $\left(P_{0} \backslash\{x, y\},\{x, s, t\}\right)$ is (still convex in $S \backslash\{y\}$ and) compatible with $\mathcal{F}_{y}$. In this case we will write $y \underset{P}{\rightarrow}$. Unlockings by compatibility are described in Subsection 5.4. 
We say that a point $x \in \mathbf{L} \cup \mathbf{U}$ is visible if $\left(P_{0} \backslash\{x\},\{x, s, t\}\right)$ is convex. The visible points of $\mathbf{L}$ are simply the extreme points of $\operatorname{conv}\left(\widetilde{P_{0}}\right)$ that are in $(s t)^{-}$. The visible points of $\mathbf{U}$, however, are not a priori in convex position.

The main idea of proof of Theorem 3.7 is to find a nonempty subset $\mathbf{T}$ of $S$, on which the relation $\rightarrow$ is bijective. We describe this set $\mathbf{T}$ in Subsection 5.6. The lengthy proof that $\rightarrow$ is a bijection on $\mathbf{T}$ is in Subsection 5.7. Then we study the orbits of this bijection and obtain the expected contradiction in Subsection 5.9.

\subsection{Description of lockings}

Firstly, the definition of locking yields immediately the following remark.

Remark 5.2. (i) Let $x$ be a visible point of $\mathbf{L}$ and let $P \in \mathcal{F}$ such that $x \in P$. If there exist $y, z \in \widetilde{P_{0}} \backslash P$, and $X \in P \backslash P_{0}$ such that $] x, X[\cap] y, z[\neq \emptyset$, i.e the quadrilateral $x y X z$ is convex, then $P$ locks $x$.

(ii) Similarly, let $X$ be a visible point of $\mathbf{U}$ and $P \in \mathcal{F}$ with $X \notin P$. If there exist $Y, Z \in P \backslash P_{0}$ and $x \in \widetilde{P_{0}} \backslash P$ such that $] x, X[\cap] Y, Z[\neq \emptyset$, i.e the quadrilateral $x Y X Z$ is convex, then $P$ locks $X$.

Conversely, we have the useful result (see Figure 8).
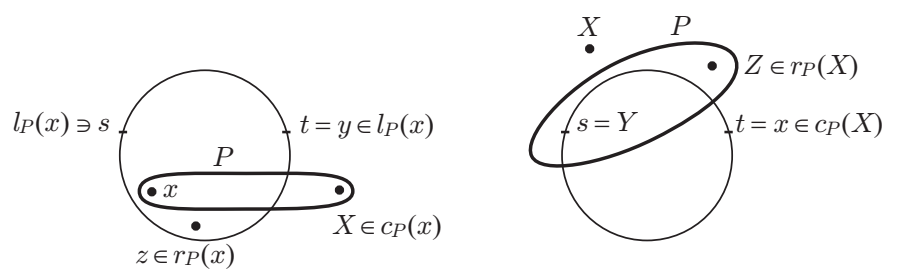

Figure 8: Examples of locking subsets of Proposition 5.3. For convenience, we draw separating curves instead of convex subsets and pairs. In all figures, we use a true circle for the pair $\left(P_{0},\{s, t\}\right)$.

Proposition 5.3. (i) If a visible point $x \in \mathbf{L}$ is locked by a subset $P \in \mathcal{F}$ then $x \in P$, $c_{P}(x)=P \backslash P_{0}$ is nonempty, and $\widetilde{P_{0}} \backslash P$ is the union of two nonempty disjoint subsets $l_{P}(x)$ and $r_{P}(x)$ such that each triple $(y, X, z)$ in $l_{P}(x) \times c_{P}(x) \times r_{P}(x)$ satisfies $y \in(x X)^{+}, z \in(x X)^{-}$, and $] x, X[\cap] y, z[\neq \emptyset$. As a consequence, one has

$$
\left(\widetilde{P_{0}} \backslash P\right) \cap(x y)^{+} \subseteq l_{P}(x) \text { for all } y \in l_{P}(x)
$$

and similarly $\left(\widetilde{P_{0}} \backslash P\right) \cap(x z)^{-} \subseteq r_{P}(x)$ for all $z \in r_{P}(x)$.

(ii) Similarly, if a visible point $X \in \mathbf{U}$ is locked by $P \in \mathcal{F}$ then $X \notin P, c_{P}(X)=\widetilde{P_{0}} \backslash P$ is nonempty, and $P \backslash P_{0}$ is the union of two nonempty disjoint subsets $l_{P}(X)$ and $r_{P}(X)$ such that each triple $(Y, x, Z)$ in $l_{P}(X) \times c_{P}(X) \times r_{P}(X)$ satisfies $Y \in(x X)^{+}, Z \in(x X)^{-}$, and ]$x, X[\cap] Y, Z\left[\neq \emptyset\right.$. As a consequence, one has $\left(P \backslash P_{0}\right) \cap(Y X)^{+} \subseteq l_{P}(X)$ for all $Y \in l_{P}(X)$ and $\left(P \backslash P_{0}\right) \cap(Z X)^{-} \subseteq r_{P}(X)$ for all $Z \in r_{P}(X)$.

We will frequently use this result, without systematically refer to it. Points in $l_{P}(x), r_{P}(x)$, and $c_{P}(x)$ are respectively called left-, right-, and central-lockers. Notice that the points $s$ and $t$ are always in at least one of the sets $l_{P}(x), c_{P}(x)$, or $r_{P}(x)$ for any visible point $x \in \mathbf{L} \cup \mathbf{U}$ and any $P \in \mathcal{F}$ locking $x$. Assertion (12) and its analogs show that this nomenclature is consistent with the usual notion of left and right. For instance, if $x \in \mathbf{L}$ is locked by $P \in \mathcal{F}$, then for all $y \in l_{P}(x)$ and all $z \in r_{P}(x)$, one has $z \in(x y)^{-}$. 
We will also use this result in the following form: If a visible point $x \in \mathbf{L}$ is locked by $P \in \mathcal{F}$, then one has

$$
\forall X \in c_{P}(x), l_{P}(x)=(x X)^{+} \cap\left(\widetilde{P_{0}} \backslash P\right) \text { and } r_{P}(x)=(x X)^{-} \cap\left(\widetilde{P_{0}} \backslash P\right) .
$$

Symmetrically, if a visible point $X \in \mathbf{U}$ is locked by $P \in \mathcal{F}$ then

$$
\forall x \in c_{P}(X), l_{P}(X)=(x X)^{+} \cap\left(P \backslash P_{0}\right) \text { and } r_{P}(X)=(x X)^{-} \cap\left(P \backslash P_{0}\right) .
$$

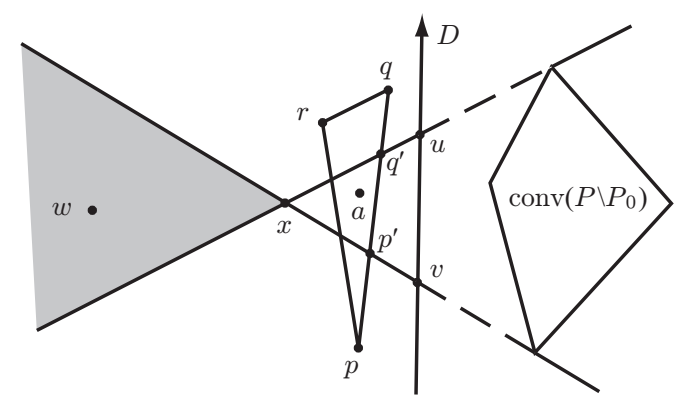

Figure 9: Proof of Proposition 5.3 (i).

Proof. (i) Since $P$ and $\left(P_{0},\{s, t\}\right)$ are compatible, there exists an oriented line $D$ containing no point in $S$ such that $\operatorname{conv}\left(P \backslash P_{0}\right) \subset D^{-}$and $\operatorname{conv}\left(\widetilde{P_{0}} \backslash P\right) \subset D^{+}$. Since $x$ is locked by $P$, the intersection $\operatorname{conv}\left(P \backslash\left(P_{0} \backslash\{x\}\right)\right) \cap \operatorname{conv}\left(\widetilde{P_{0}} \backslash P\right)$ is nonempty. It follows that $x$ is in $P$ but not in $D^{-}$and that $P \backslash\left(P_{0} \backslash\{x\}\right)=\left(P \backslash P_{0}\right) \cup\{x\}$. Therefore, the set $\Delta=\bar{D}^{+} \cap \operatorname{conv}\left(\left(P \backslash P_{0}\right) \cup\{x\}\right)$ is a nonempty triangle or a line segment that contains at least one point $a$ of $\operatorname{conv}\left(\widetilde{P_{0}} \backslash P\right)$, see Figure 9. The vertices of $\Delta$ are the point $x$ and one or two other points $u$ and $v$ lying on $D$. We can suppose that $u \in \overline{(x v)^{+}}$. Observe that $\Delta$ cannot contain a point in $\widetilde{P_{0}} \backslash P$ because such a point would be in $S \cap \operatorname{conv}\left(\left(P \backslash P_{0}\right) \cup\{x\}\right)$ which is included in $S \cap \operatorname{conv}(P)=P$. Therefore, the point $a$ is in a triangle (possibly reduced to a segment) with vertices $p, q, r$ in $\widetilde{P_{0}} \backslash P$, none of which being in $\Delta$. It follows that one side of this triangle, say $] p, q[$, must cut the (possibly identical) open sides $] x, u[$ and $] x, v\left[\right.$ of $\Delta$, at some points denoted $p^{\prime}$ and $q^{\prime}$. Moreover, by Remark 5.1, for any $w$ in the cone $\overline{(x u)^{+}} \cap \overline{(x v)^{-}}, x$ is in the triangle $p^{\prime} q^{\prime} w$, hence in the triangle $p q w$. As a consequence, since $x$ is an extreme point of $\operatorname{conv}\left(\widetilde{P}_{0}\right)$, no point of $\operatorname{conv}\left(\widetilde{P_{0}} \backslash P\right)$ can be in $\overline{(x u)^{+}} \cap \overline{(x v)^{-}}$. It follows that the two subsets $l_{P}(x)=\left(\widetilde{P_{0}} \backslash P\right) \cap \overline{(x u)^{+}}$ and $r_{P}(x)=\left(\widetilde{P_{0}} \backslash P\right) \cap \overline{(x v)^{-}}$form a partition of $\widetilde{P_{0}} \backslash P$. Each of these two subsets is nonempty because one of them contains $p$ and the other one contains $q$. Finally, consider a line segment $[y, z]$ with $y \in l_{P}(x)$ and $z \in r_{P}(x)$. Since it does not meet the cone $\overline{(x u)^{+}} \cap \overline{(x v)^{-}}$, the open segment $] y, z[$ meets $] x, u[$ and $] x, v\left[\right.$. It follows that, for all $\left.X \in c_{P}(x)=P \backslash P_{0},\right] y, z[\cap] x, X[$ is nonempty.

For a proof of (12), choose $X \in c_{P}(x)$; Since $y \in(x X)^{+}$, we have $X \in(x y)^{-}$. As a consequence, for any $w \in\left(\widetilde{P_{0}} \backslash P\right) \cap(x y)^{+}$we have $] x, X[\cap] y, w\left[=\emptyset\right.$, showing that $w \notin r_{P}(x)$, hence $w \in l_{P}(x)$.

(ii) This case is very similar but we write its proof for completeness. Since $P$ and $\left(P_{0},\{s, t\}\right)$ are compatible, there exists an oriented line $D$, containing no point in $S$, such that $\operatorname{conv}\left(P \backslash P_{0}\right) \subset$ $D^{-}$and $\operatorname{conv}\left(\widetilde{P_{0}} \backslash P\right) \subset D^{+}$. Since $X$ is locked by $P, \operatorname{conv}\left(P \backslash P_{0}\right) \cap \operatorname{conv}\left(\left(\widetilde{P_{0}} \cup\{X\}\right) \backslash P\right)$ is nonempty. Therefore $X$ is neither in $P$ nor in $D^{+}$, hence $\left(\widetilde{P_{0}} \cup\{X\}\right) \backslash P=\left(\widetilde{P_{0}} \backslash P\right) \cup\{X\}$ and $X \in D^{-}$. It follows that the intersection $\operatorname{conv}\left(\left(\widetilde{P_{0}} \backslash P\right) \cup\{X\}\right) \cap \bar{D}^{-}$is a triangle or a line segment $\Delta=\operatorname{conv}(\{X, u, v\}\}$ with $u$ and $v$ lying in $D$. We can suppose that $u \in \overline{(X v)^{+}}$. Since $\operatorname{conv}\left(P \backslash P_{0}\right)$ is included in $D^{-}$and meets $\operatorname{conv}\left(\left(\widetilde{P_{0}} \backslash P\right) \cup\{X\}\right), \Delta$ contains at least a point $a$ which belongs to $\operatorname{conv}\left(P \backslash P_{0}\right)$. If $\Delta$ contained a point $p$ of $P \backslash P_{0}$, this point $p$ would be in 
$\operatorname{conv}\left(\left(\widetilde{P_{0}} \backslash P\right) \cup\{X\}\right)$ which is included in $\operatorname{conv}\left(\widetilde{P_{0}} \cup\{X\}\right)$. Since the pair $\left(P_{0},\{X, s, t\}\right)$ is convex, we would have $p \in \widetilde{P_{0}} \cup\{X\}$ and thus $p=X$, s or $t$. The case $p=X$ cannot occur because $X \notin P$. The case $p=s$ or $t$, say $s$, implies $s \in \operatorname{conv}\left(\left(\widetilde{P_{0}} \backslash P\right) \cup\{X\}\right) \cap D^{-}$. Since $s \in P$, this implies that $s$ would not be extreme in $\left(P_{0} \cup\{X\}\right) \backslash P$, i.e. $\left(P_{0},\{X, s, t\}\right)$ would not be convex, contradicting the visibility of $X$. Therefore the point $a$ is in a triangle (possibly reduced to a segment) with vertices $p, q$ and $r$ in $P \backslash P_{0}$, none of which being in $\Delta$. It follows that one side of this triangle, say $[p, q]$, must cut the (possibly identical) open sides $] X, u[$ et $] X, v[$ of $\Delta$. The fact that $X$ is not in $P$ then implies that the cone $(X u)^{+} \cap(X v)^{-}$does not meet $\operatorname{conv}\left(P \backslash P_{0}\right)$. It follows that the two subsets $l_{P}(X)=\left(P \backslash P_{0}\right) \cap(X u)^{+}$and $r_{P}(X)=\left(P \backslash P_{0}\right) \cap(X v)^{-}$form a partition of $P \backslash P_{0}$. The points $p$ and $q$ are neither both in $l_{P}(X)$ nor both in $r_{P}(X)$ because the segment $[p, q]$ meets the two open segments $] X, u[$ and $] X, v\left[\right.$. Therefore $l_{P}(X)$ and $r_{P}(X)$ are nonempty. Finally, let $Y \in l_{P}(X)$ and $Z \in r_{P}(X)$. Since the cone $(X u)^{+} \cap(X v)^{-}$does not intersect $\operatorname{conv}\left(P \backslash P_{0}\right)$, the segment $] Y, Z[$ must cut the open sides $] X, u[$ of $] X, v[$ of the triangle $\Delta$. It follows that $] Y, Z[$ crosses every segment $] X, x\left[\right.$ with $x \in c_{P}(X)$.

\subsection{Unlockings by compatibility}

From Remark 5.2 and Proposition 5.3, if a point $y \in S \backslash\{s, t\}$ unlocks a point $x \in \mathbf{L} \cup \mathbf{U}$ by compatibility, then there exists $P \in \mathcal{F}$ such that $\{y\}$ is one of the sets $l_{P}(x), c_{P}(x)$, or $r_{P}(x)$. For any $x \in \mathbf{L} \cup \mathbf{U}$, we consider the three unions

$$
l(x)=\bigcup_{P \in \mathcal{F}} l_{P}(x), \quad c(x)=\bigcup_{P \in \mathcal{F}} c_{P}(x), \quad r(x)=\bigcup_{P \in \mathcal{F}} r_{P}(x)
$$

with the convention $l_{P}(x)=c_{P}(x)=r_{P}(x)=\emptyset$ if $P$ does not lock $x$. Observe that, if $x \in \mathbf{L}$, then $l(x) \cup r(x) \subseteq \widetilde{P_{0}}$ and $c(x) \subseteq S \backslash P_{0}$. Symmetrically, if $x \in \mathbf{U}$, then $l(x) \cup r(x) \subseteq S \backslash P_{0}$ and $c(x) \subseteq \widetilde{P_{0}}$. For a fixed $P$ the sets $l_{P}(x), c_{P}(x)$, and $r_{P}(x)$ are disjoint, but this is no longer true a priori for the unions $l(x), c(x)$, and $r(x)$.

In this section, we prove that, if $y$ unlocks $x$, then firstly $y$ can be in only one of the three sets $l(x), c(x)$, and $r(x)$, and secondly $y$ is alone in this set.

Proposition 5.4. If a point $y \in S \backslash\{s, t\}$ unlocks a visible point $x \in \mathbf{L} \cup \mathbf{U}$ by compatibility, then $y$ is in only one of the three sets $l(x), c(x)$, or $r(x)$.

Proof. We consider four different cases, whether $x$ is in $\mathbf{L}$ or in $\mathbf{U}$ and whether $y$ is or is not in $P_{0}$.

The cases $x \in \mathbf{L}, y \notin P_{0}$ and $x \in \mathbf{U}, y \in P_{0}$ are evident: In these cases $y$ cannot be in $l(x) \cup r(x)$; It has to be only in $c(x)$.

If $x \in \mathbf{L}$ and $y \in P_{0}$, assume by contradiction that there exist $P_{1}, P_{2} \in \mathcal{F}$ such that $y \in l_{P_{1}}(x) \cap r_{P_{2}}(x)$. By Proposition 5.3 (i), there exist $X_{1} \in c_{P_{1}}(x), X_{2} \in c_{P_{2}}(x), z_{1} \in r_{P_{1}}(x)$, and $z_{2} \in l_{P_{2}}(x)$ such that both intersections $] x, X_{1}[\cap] y, z_{1}[$ and $] x, X_{2}[\cap] y, z_{2}[$ are nonempty, see Figure 10 left. The idea is to prove that $z_{2} \in l_{P_{1}}(x)$. Since $y \in\left(x X_{1}\right)^{+}$, we have $X_{1} \in(x y)^{-}$. Since $] x, X_{1}[$ and $] y, z_{1}\left[\right.$ cross, we then have $z_{1} \in(x y)^{-}$. Similarly, we have $z_{2} \in(x y)^{+}$.

If $z_{2} \in\left(x z_{1}\right)^{-}$then $z_{2} \in(x y)^{+} \cap\left(x z_{1}\right)^{-}$. Remark 5.1 then yields $x \in \operatorname{conv}\left(\left\{y, z_{1}, z_{2}\right\}\right)$, in contradiction with the fact that $x$ is an extreme point of $\widetilde{P_{0}}$. Therefore $z_{2} \in\left(x z_{1}\right)^{+}$. It follows that the points $X_{1}, y, X_{2}$, and $z_{2}$ are all in the half plane $\left(x z_{1}\right)^{+}$(for $X_{2}$, this follows from $\left.X_{2} \in(x y)^{+} \cap\left(x z_{2}\right)^{-}\right)$. Now $y \in\left(x X_{1}\right)^{+}, X_{2} \in(x y)^{+}$, and $z_{2} \in\left(x X_{2}\right)^{+}$, hence the segment ]$z_{1}, z_{2}$ [ meets the half line $\left(x X_{1}\right) \cap\left(x z_{1}\right)^{+}$in a point $X_{1}^{\prime}$. Since $X_{1}$ is not in $\operatorname{conv}\left(\left\{z_{1}, z_{2}, x\right\}\right)$, we have $\left.X_{1}^{\prime} \in\right] x, X_{1}\left[\right.$. This proves that $z_{2} \in l_{P_{1}}(x)$. Therefore removing $y$ would not unlock the point $x$.

If $x \in \mathbf{U}$ and $y \notin P_{0}$, assume by contradiction that there exist $P_{1}, P_{2} \in \mathcal{F}$ such that $y \in l_{P_{1}}(x) \cap r_{P_{2}}(x)$. By Proposition 5.3 (ii), there exist $x_{1} \in c_{P_{1}}(x), Z_{1} \in r_{P_{1}}(x), Z_{2} \in l_{P_{2}}(x)$ 

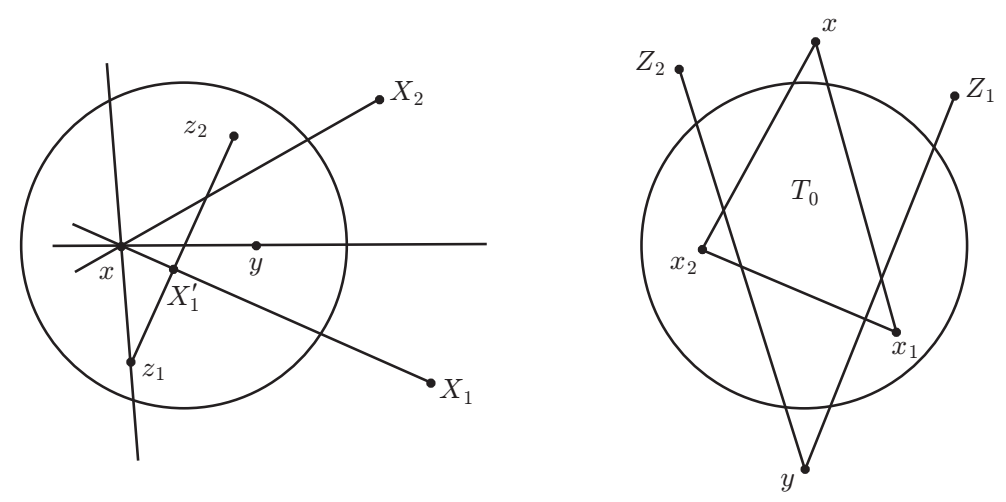

Figure 10: Proof of Proposition 5.4.

and $x_{2} \in c_{P_{2}}(x)$ such that both intersections $] x, x_{1}[\cap] y, Z_{1}[$ and $] x, x_{2}[\cap] y, Z_{2}[$ are nonempty. Observe that, since $x_{1}, Z_{1} \in(y x)^{+}$and $x_{2}, Z_{2} \in(y x)^{-}$, one has $x_{1} \neq x_{2}$ and $Z_{1} \neq Z_{2}$.

Consider the triangle $T_{0}=\operatorname{conv}\left(\left\{x, x_{1}, x_{2}\right\}\right) \subseteq \operatorname{conv}\left(\widetilde{P_{0}} \cup\{x\}\right)$. Since $y \notin \widetilde{P_{0}}$ and since the pair $\left(P_{0},\{x, s, t\}\right)$ is convex, the point $y$ cannot be in $T_{0}$. We claim that $Z_{1} \notin T_{0}$. Indeed, if $Z_{1} \in T_{0}$, since $Z_{1} \notin P_{0}$ the only possibility is $Z_{1}=x_{2}\left(=s\right.$ or $t$ ). Since $Z_{1} \in\left(x_{1} x\right)^{-}$, this implies $x_{1} \in\left(x_{2} x\right)^{+}$. Therefore the segment $\left[x_{2}, y\right]=\left[Z_{1}, y\right]$, which meets a point of $\left[x, x_{1}\right]$, is included in $\left(x_{2} x\right)^{+}$. It follows that $y \in\left(x_{2} x\right)^{+}$contradicting $y \in r_{P_{2}}(x)$. In the same way we have $Z_{2} \notin T_{0}$. Now the proof splits in two subcases.

If $x_{2} \in\left(x_{1} x\right)^{-}$, then the points $y$ and $x_{1}$ are on each side of the line $\left(x_{2} x\right)$. The segment $] y, Z_{2}[$, which cuts the segment $] x, x_{2}\left[\right.$, must cross another side of the triangle $T_{0}$. Since $] x_{1}, x\left[\subset(y x)^{-}\right.$ and $] y, Z_{2}\left[\subset(y x)^{+}\right.$, it has to cross the side $] x_{1}, x_{2}$ [ of $T_{0}$. Besides, if $x_{1}$ were in $P_{2}$, we would have $x \in \operatorname{conv}\left(y, x_{1}, Z_{2}\right) \subseteq \operatorname{conv}\left(P_{2}\right)$, a contradiction, hence $x_{1} \notin P_{2}$. To sum up, we have $x_{1}, x_{2} \in \widetilde{P_{0}} \backslash P_{2}, y, Z_{2} \in P_{2} \backslash P_{0}$, and $] y, Z_{2}[\cap] x_{1}, x_{2}[\neq \emptyset$, contradicting the compatibility of $\left(P_{0},\{s, t\}\right)$ and $P_{2}$.

The second subcase is $x_{2} \in\left(x_{1} x\right)^{+}$, see Figure 10 right. We split the proof in four steps.

Step 1. We have again $] y, Z_{2}[\cap] x_{1}, x_{2}\left[\neq \emptyset\right.$. The assumption $x_{2} \in\left(x_{1} x\right)^{+}$implies that the triangle $T_{0}$ is included in the cone $\left(x_{1} x\right)^{+} \cap\left(x_{2} x\right)^{-}$. The point $y$ is also in this cone while $Z_{2}$ is not. Therefore one of the two sides of $T_{0}$ that are crossed by the segment ] $y, Z_{2}$ [, must be ]$x_{1}, x_{2}[$.

Step 2. We have $x_{1} \in P_{2}$. Otherwise $\left[x_{1}, x_{2}\right]$ is included in $\operatorname{conv}\left(\widetilde{P_{0}} \backslash P_{2}\right)$ and, together with the first step, this implies that $\left(P_{0},\{s, t\}\right)$ and $P_{2}$ are incompatible. The same argument shows that $x_{2} \in P_{1}$ as well.

Step 3. We have $Z_{1} \in P_{2}$ or $Z_{2} \in P_{1}$. Observe first that $Z_{2}$ is in $\left(x_{1} x\right)^{+}$. Otherwise the point $x$ would be in the triangle $\operatorname{conv}\left(\left\{y, Z_{2}, x_{1}\right\}\right)$ which is included in $P_{2}$, a contradiction. The same argument shows that $Z_{1} \in\left(x_{2} x\right)^{-}$. Since $y$ is not in $T_{0}, Z_{1}$ and $Z_{2}$ are in $\left(x_{1} x_{2}\right)^{-}$. Therefore $Z_{1} \in\left(x_{2} x_{1}\right)^{+} \cap\left(x_{2} x\right)^{-}$and $Z_{2} \in\left(x_{1} x_{2}\right)^{-} \cap\left(x_{1} x\right)^{+}$. It follows that the segments $\left[x_{1}, Z_{2}\right]$ and $\left[x_{2}, Z_{1}\right]$ intersect. Since $x_{1} \in P_{2} \backslash P_{1}, x_{2} \in P_{1} \backslash P_{2}, Z_{1} \in P_{1}$ and $Z_{2} \in P_{2}$, it is not possible that both $Z_{1} \notin P_{2}$ and $Z_{2} \notin P_{1}$ because this would imply that $P_{1}$ and $P_{2}$ are incompatible.

Step 4. To fix ideas, we suppose that $Z_{1} \in P_{2}$. We have proven that $Z_{1} \in\left(x_{2} x\right)^{-}$and since $Z_{1} \notin P_{0}$ this means that $Z_{1} \in r_{P_{2}}(x)$. Therefore $y$ and $Z_{1}$ are both in $r_{P_{2}}(x)$ which implies that $y$ cannot unlock $x$.

Corollary 5.5. If a point $y \in S \backslash\{s, t\}$ unlocks a visible point $x \in \mathbf{L} \cup \mathbf{U}$ by compatibility, then $y$ is the only point of one of the three categories $l(x), c(x)$, or $r(x)$.

Proof. Suppose $y$ unlocks $x$ and let $P_{1} \in \mathcal{F}$ be such that $y \underset{P_{1}}{\rightarrow}$. One of the sets $l_{P_{1}}(x), c_{P_{1}}(x)$, or $r_{P_{1}}(x)$ is reduced to $y$. To fix ideas, assume that $l_{P_{1}}(x)=\{y\}$. Then by Proposition 5.4, for 
all $P$ locking $x$, we have $y \in l_{P}(x)$, and $c_{P}(x)$ and $r_{P}(x)$ nonempty. Because $y$ unlocks $x$, we then have $l_{P}(x)=\{y\}$ for all $P$ locking $x$, hence $l(x)=\{y\}$.

\subsection{Unlockings by convexity}

Given $X \in S \backslash \widetilde{P_{0}}$, we call cone of $X$ the set $C(X)=\operatorname{relint}\left(\operatorname{conv}\left(\widetilde{P_{0}} \cup\{X\}\right) \backslash \operatorname{conv}\left(\widetilde{P_{0}}\right)\right)$. In other words, $C(X)$ is the truncated open cone between $X$ and $\operatorname{conv}\left(\widetilde{P_{0}}\right)$. Notice that, if $Y \in C(X) \cap S$, then $C(Y) \subset C(X)$.

Given $X \in S \backslash \widetilde{P_{0}}$ and $x \in P_{0}$, let $C_{x}(X)=\operatorname{relint}\left(\operatorname{conv}\left(\left(\widetilde{P_{0}} \cup\{X\}\right) \backslash\{x\}\right) \backslash\left(\operatorname{conv}\left(\widetilde{P_{0}}\right) \backslash\{x\}\right)\right)$ denote the cone of $X$ relatively to $P_{0} \backslash\{x\}$.

If $X \in \mathbf{U}$ is invisible, i.e. $\left(P_{0},\{X, s, t\}\right)$ is nonconvex, then at least one of the two cases below occurs. Either conv $\left(\widetilde{P_{0}} \cup\{X\}\right) \cap S$ strictly contains $\widetilde{P_{0}} \cup\{X\}$, hence $C(X) \cap S$ contains some $Y$, see Figure 11 left. Or $s$ and $t$ are not both extreme points of $\widetilde{P_{0}} \cup\{X\}$, hence there is $y \in \mathbf{L}$ such that $X \in(y s)^{+} \cup(y t)^{-}$, see Figure 11 right. In both cases we say that $Y$ or $y$ hides $X$. Moreover, if the second case does not occur, i.e. if $s$ and $t$ are both extreme points of $\widetilde{P_{0}} \cup\{X\}$, then the point $Y$ which hides $X$ is necessarily in $\mathbf{U}$.
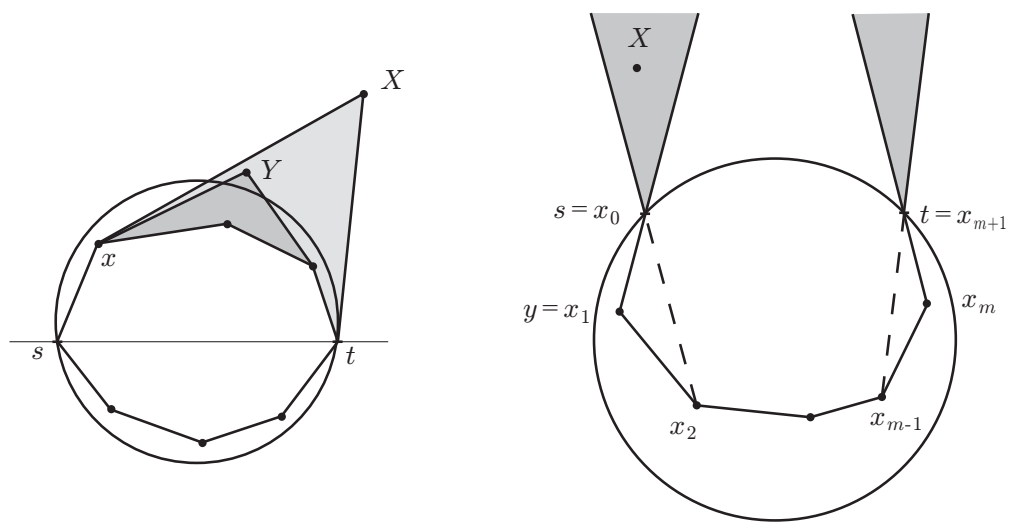

Figure 11: Left, in light gray $C(X)$, in dark gray $C(Y)$. Right, in gray $V\left(x_{2}\right) \backslash V\left(x_{1}\right)$ and $V\left(x_{m-1}\right) \backslash V\left(x_{m}\right)$.

For any $z \in \mathbf{L}$, let $V(z)=(z s)^{-} \cap(z t)^{+} \cap(s t)^{+}$. Hence $V(z) \cap S$ is the set of points of $\mathbf{U}$ that are not hidden by $z$. Observe that, for all $u, v, w \in \mathbf{L}$,

$$
\text { if } z \in \operatorname{conv}(\{u, v, w\}) \text { then } V(z) \subseteq V(u) \cup V(v) \cup V(w) .
$$

If a point $X \in \mathbf{U}$ is unlocked by convexity, then this can be done in three different ways, see Figure 11:

1. Either $X$ is unlocked by a point $Y \in S \backslash \widetilde{P_{0}}$, if $C(X) \cap S=\{Y\}$,

2. or by a point $x \in P_{0}$, if $C(X) \cap S$ is nonempty, but $C_{x}(X) \cap S$ is empty,

3. or by a point $y \in \mathbf{L}$, if $X$ is not in $V(y)$ but is in $V(x)$ for all $x \in \mathbf{L} \backslash\{y\}$.

Cases 1 and 2 can occur simultaneously, as well as cases 2 and 3 . In the first two cases, we necessarily have $C(X) \subset(s t)^{+}$, otherwise $X$ would be locked by convexity by a point of $\mathbf{L}$. In the third case, we can be more precise: Let $s=x_{0}<x_{1}<\cdots<x_{m}<x_{m+1}=t$ denote the points of $\partial \operatorname{conv}\left(\widetilde{P_{0}}\right) \cap \overline{(s t)^{-}}$in their natural order. Then, from (13) and since $V\left(x_{i}\right) \subseteq V\left(x_{1}\right) \cup V\left(x_{m}\right)$ for all $i \in\{1, \ldots, m\}$, we have the following remark, see Figure 11 right.

Remark 5.6. For $m \geq 2$, if $X \in \mathbf{U}$ is unlocked by convexity by $y \in \mathbf{L}$, then either $y=x_{1}$ and $X \in V\left(x_{2}\right) \backslash V\left(x_{1}\right)$, or $y=x_{m}$ and $X \in V\left(x_{m-1}\right) \backslash V\left(x_{m}\right)$.

For $m=1$, if $X \in \mathbf{U}$ is unlocked by convexity by $y \in \mathbf{L}$, then $y=x_{1}$ and $X \notin V\left(x_{1}\right)$. 
The next proposition eliminates most of the possibilities to unlock a point by convexity.

Proposition 5.7. (i) All points of $\mathbf{L}$ are visible, i.e. $\mathbf{L} \subset \partial \operatorname{conv}\left(\widetilde{P_{0}}\right)$.

(ii) An invisible point of $\mathbf{U}$ can be unlocked (by convexity) only by a point of $P_{0}$.

Proof. (i) By contradiction let $y \in \mathbf{L}$ be invisible, i.e. not an extreme point of $\widetilde{P_{0}}$. Let $u, v, w \in$ $\widetilde{P_{0}}$ be extreme and such that $y \in \operatorname{conv}(\{u, v, w\})$. By (11), there exists $x \in \mathbf{L} \cup \mathbf{U}$ such that $y$ unlocks $x$.

Assume first that $x \in \mathbf{U}$. If $y$ hid $x$, then by (13) $x$ would be hidden by one of the points $u, v$ or $w$, preventing $y$ to unlock $x$. Hence $y$ unlocks $x$ by compatibility: There exists $P \in \mathcal{F}$ such that $\{y\}=c_{P}(x)=\widetilde{P_{0}} \backslash P$. This implies $u, v, w \in P$, hence $y \in P$ by convexity of $P$, a contradiction.

Now suppose $x \in \mathbf{L}$. As before, $y$ cannot hide $x$ : Indeed, since $y$ is not extreme in $\widetilde{P_{0}}$ and $x$ is extreme in $\widetilde{P_{0}} \backslash\{y\}, x$ is extreme in $\widetilde{P_{0}}$. By Corollary 5.5 we thus have $l(x)$ or $r(x)=\{y\}$. Assume $l(x)=\{y\}$ to fix ideas. Let $P \in \mathcal{F}$ be such that $y \underset{P}{\rightarrow} x, X \in c_{P}(x)$, and $z \in r_{P}(x)$ (hence $] x, X[\cap] y, z[\neq \emptyset)$. The set $\operatorname{relint}(\operatorname{conv}(\{u, v, w, z\}))$ contains neither $x$ (which is an extreme point of $\widetilde{P_{0}}$ ) nor $X$ (since $X \notin \widetilde{P_{0}}$ ). As a consequence, $] x, X[\operatorname{cuts} \operatorname{conv}(\{u, v, w, z\})$ in two connected components, one containing $z$, the other containing $y$ and at least one of the points $u, v$ or $w$.

If the component containing $y$ contains one point, say $u$, then we have $x, X \in P, y \in$ $\operatorname{conv}(u, x, X) \backslash P$, hence $u \notin P$ by convexity of $P$. If it contains two points $u, v$, then by the same reason, since $y \in \operatorname{conv}(u, v, x, X)$, one of the points, say $u$, is not in $P$. see Figure 12 left. This yields $] x, X[\cap] u, z\left[\neq \emptyset\right.$, hence $u \in l_{P}(x)$, a contradiction with $l(x)=\{y\}$.
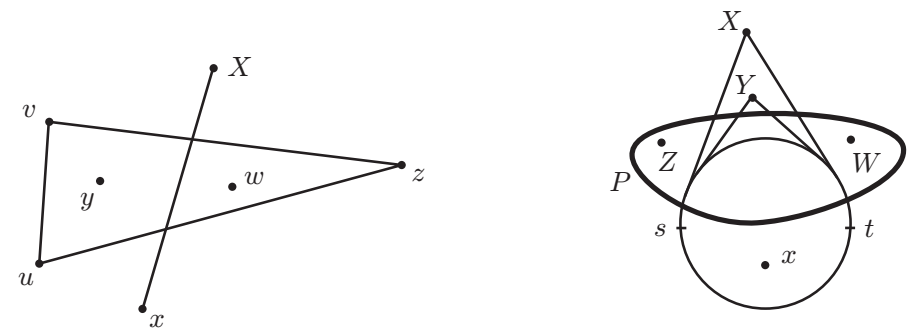

Figure 12: Proof of Proposition 5.7.

(ii) Again by contradiction, let $X \in \mathbf{U}$ be unlocked by convexity by $Y \in S \backslash \widetilde{P_{0}}$, hence $C(X) \cap S=$ $\{Y\}$. If $Y$ were not in $\mathbf{U}$, then $X$ would also be locked by convexity by a point of $\mathbf{L}$, hence not unlocked by $Y$. Therefore $Y \in \mathbf{U}$. Since $Y \in C(X)$, we have $C(Y) \subset C(X)$, hence $C(Y) \cap S=\emptyset$, i.e. $Y$ cannot be hidden by a point of $S \backslash \widetilde{P_{0}}$. However $Y$ has to be locked.

If $Y$ were locked by convexity then, since $C(Y)=\emptyset, Y$ would be hidden by some point of L. This means that $s$ or $t$ would not be extremal in $\operatorname{conv}\left(\widetilde{P_{0}} \cup\{Y\}\right)$. Since $\operatorname{conv}\left(\widetilde{P_{0}} \cup\{Y\}\right) \subset$ $\operatorname{conv}\left(\widetilde{P_{0}} \cup\{X\}\right), s$ or $t$ would not be extremal in $\operatorname{conv}\left(\widetilde{P_{0}} \cup\{X\}\right)$, too, hence $X$ would be locked by convexity, contradicting $Y \rightarrow X$.

As a consequence, $Y$ is locked by compatibility, by some subset $P \in \mathcal{F}$. Let $Z \in l_{P}(Y)$, $x \in c_{P}(Y)$, and $W \in r_{P}(Y)$, see Figure 12 right. Let $u, v \in \widetilde{P_{0}}$ be such that $Y \in \operatorname{conv}(u, v, X)$. The segment $] Z, W$ [ crosses $] x, Y$ [, which is included in the interior of $\operatorname{conv}(u, v, x, X)$, while neither $Z$ nor $W$ is in the interior of $\operatorname{conv}(u, v, x, X)$ because $C(X) \cap S=\{Y\}$. It follows that $] Z, W\left[\right.$ crosses the boundary of $\operatorname{conv}(u, v, x, X)$ in two points $\left.Z^{\prime}, W^{\prime} \in\right] Z, W[$, and cuts $\operatorname{conv}(u, v, x, X)$ in two connected components: $\Omega^{+}=\operatorname{conv}(u, v, x, X) \cap(Z W)^{+}$and $\Omega^{-}=$ $\operatorname{conv}(u, v, x, X) \cap(Z W)^{-}$. Since $Z \in l_{P}(Y), x \in c_{P}(Y)$, and $W \in r_{P}(Y)$, we have $Y \in \Omega^{+}$and $x \in \Omega^{-}$. Since $Z, W \in P$, we have $] Z^{\prime}, W^{\prime}[\subset \operatorname{conv}(P)$. 
If $X$ were in $\Omega^{-}$, we would have $Y \in \operatorname{conv}\left(\Omega^{+} \cap\{u, v\}, Z^{\prime}, W^{\prime}\right)$, which is included in $\operatorname{conv}(P)$, a contradiction with $Y \notin P$. Therefore $X \in \Omega^{+}$. If $X$ were in $P$, we would have $Y \in$ $\operatorname{conv}\left(\Omega^{+} \cap\{u, v\}, Z^{\prime}, W^{\prime}, X\right) \subset \operatorname{conv}(P)$, again a contradiction with $Y \notin P$. Therefore $X \notin P$.

Now $x \in \Omega^{-}$and $X \in \Omega^{+}$imply that ] $x, X[$ crosses $] Z, W$, which implies that $P$ locks $X$, a contradiction with $Y \rightarrow X$.

Proposition 5.8. If $\mathbf{L} \cup \mathbf{U}=\{r\}$, then $\left(P_{0} \backslash\{r\},\{r, s, t\}\right)$ is compatible with $\mathcal{F}$.

Proof. By contradiction, assume $\mathbf{L} \cup \mathbf{U}=\{r\}$ and $r$ locked. If $r \in \mathbf{L}$ then $r$ is an extreme point of $P_{0}$, hence locked by compatibility, by some $P \in \mathcal{F}$ containing it. From Proposition 5.3 (i), there exist $X \in P \backslash P_{0}$ and $y, z \in \widetilde{P_{0}} \backslash P$ such that $] r, X[\cap] y, z[\neq \emptyset$. Since $\mathbf{L}=\{r\} \subseteq P$, one

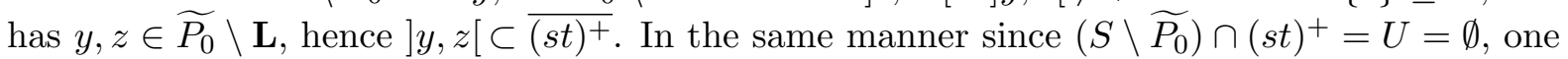
has $X \notin(s t)^{+}$, hence $] r, X\left[\subset(s t)^{-}\right.$. This contradicts $] r, X[\cap] y, z[\neq \emptyset$.

The case $r \in \mathbf{U}$ is analogous: $\mathbf{L} \cup \mathbf{U}=\{r\}$ implies that the pair $\left(P_{0},\{r, s, t\}\right)$ is convex, hence $r$ is locked by compatibility, by some $P \in \mathcal{F}$. Proposition 5.3 (ii) yields $Y, Z \in P \backslash P_{0}$ and $x \in \widetilde{P_{0}} \backslash P$ such that $] r, x[\cap] Y, Z\left[\neq \emptyset\right.$. However $\mathbf{L} \cup \mathbf{U}=\{r\}$ implies firstly $x \in \widetilde{P_{0}} \backslash \mathbf{L}$, hence ]$r, x\left[\subset(s t)^{+}\right.$, and secondly $Y, Z \notin \mathbf{U} \cup P_{0}$, hence $] Y, Z\left[\subset \overline{(s t)^{-}}\right.$, contradicting $] r, x[\cap] Y, Z[\neq$ $\emptyset$.

\subsection{Ordering of unlockable points}

By Proposition 5.7 (i), we have $\mathbf{L}=\partial \operatorname{conv}\left(\widetilde{P_{0}}\right) \cap(s t)^{-}=\left\{x_{1}, \ldots, x_{m}\right\}$.

Let $\mathbf{K}=\left\{X \in \mathbf{U} \cap V\left(x_{2}\right) \cap V\left(x_{m-1}\right) ; C(X) \cap S=\emptyset\right\}$ if $m \geq 2$, and $\mathbf{K}=\{X \in$ $\mathbf{U} ; C(X) \cap S=\emptyset\}$ otherwise. As a consequence, if a point of $\mathbf{U}$ is not hidden by a point of $S \backslash \widetilde{P_{0}}$ and if it is hidden by at most one point of $\mathbf{L}$, then this point must be in $\mathbf{K}$. Observe that there might be points of $\mathbf{K}$ hidden by two points of $\mathbf{L}$ (namely $x_{1}$ and $x_{m}$ ) in the case where $\left(V\left(x_{2}\right) \backslash V\left(x_{1}\right)\right) \cap\left(V\left(x_{m-1}\right) \backslash V\left(x_{m}\right)\right)$ is non-empty. Now we set $\mathbf{T}=\mathbf{L} \cup \mathbf{K}$. By Remark 5.6 and Proposition 5.7 (ii), if a point of $x \in \mathbf{L} \cup \mathbf{U}$ is unlocked by a point $y \in \mathbf{L} \cup \mathbf{U}$, then necessarily $x \in \mathbf{T}$. In other words, one has

$$
\forall y \in \mathbf{L} \cup \mathbf{U}, \quad \emptyset \neq D(y) \subseteq \mathbf{T} .
$$

The order on $\mathbf{L}$ is the natural order $x_{1}<\cdots<x_{m}$. On $\mathbf{K}$, we define the following order: For $X \neq Y \in \mathbf{K}$, we say that $X$ is on the left of $Y$, and we denote $X<Y$ or $Y>X$, if $Y \in(s X)^{-}$. Because $\mathbf{K} \subseteq(s t)^{+}$, the relation $X \leq Y$ if $X=Y$ or $X<Y$ is a total order on $\mathbf{K}$. Notice that, if we replace $s$ by $t$, then this defines the same order on $\mathbf{K}$. Actually, if $Y \in(s X)^{+} \cap(t X)^{-}$ then $X \in C(Y)$, which is excluded for points of $\mathbf{K}$. Notice also that $X<Y$ is equivalent to $[s, X] \cap[t, Y]=\emptyset$ and also equivalent to $] s, Y[\cap] t, X[\neq \emptyset$. We will also use the following fact:

$$
\text { If } x, y \in \widetilde{P_{0}}, X \in S \backslash \widetilde{P_{0}} \text {, and } Y \in \mathbf{K} \backslash\{X\} \text {, then } X \notin \operatorname{conv}(x, y, Y) \text {. }
$$

This comes from the fact that, otherwise, $X$ would hide $Y$.

A priori there is no connection between the notions of right and left for lockers and for the order on $\mathbf{L}$ : Given two points $x, y \in \mathbf{L}$, it is possible to have at the same time $x<y$ and $y \in l(x)$. Nevertheless, the following lemma shows that this connection exists if $y$ unlocks $x$. The same is true for points $X, Y \in \mathbf{K}$.

Lemma 5.9. (i) Let $x \neq y$ be in $\mathbf{L}$ and let $P \in \mathcal{F}$ locking $x$. If $l_{P}(x)=\{y\}$ then $y<x$. In particular and by contraposition, if $y$ unlocks $x$ and $x<y$, then $r(x)=\{y\}$. Similarly, $r_{P}(x)=\{y\}$ implies $x<y$ and $(y \rightarrow x$ and $y<x)$ implies $l(x)=\{y\}$.

(ii) Let $X \neq Y$ be in $\mathbf{K}$ and let $P \in \mathcal{F}$ locking $X$. If $l_{P}(X)=\{Y\}$ then $Y<X$. In particular, if $Y$ unlocks $X$ and $X<Y$, then $r(X)=\{Y\}$. As in (i), the same holds with left and right switched. 
Proof. (i) The assumption $l_{P}(x)=\{y\}$ implies $s \in c_{P}(x) \cup r_{P}(x)$. It follows that $y \in(x s)^{+}$ which means $y<x$. By contraposition, if $y$ unlocks $x$ and $r(x) \neq\{y\}$ then, because $y \in \mathbf{L}$, we would have $\{y\}=l(x)$, hence $l_{P}(x)=\{y\}$ for any $P \in \mathcal{F}$ locking $x$, yielding $y<x$. The proof of (ii) is similar.

\subsection{The bijection}

The reason to introduce $\mathbf{T}$ lies in the following crucial result.

Theorem 5.10. If $y$ and $z$ are distinct points of $\mathbf{T}$, then $D(y)$ and $D(z)$ are nonempty disjoint subsets of $\mathbf{T}$.

Proof. We proceed by contradiction. Let $x, y, z \in \mathbf{T}$ be such that $x \in D(y) \cap D(z)$. If the point $x$ were hidden by one of the points, say $y$ (or by both), then $z$ would not unlock $x$. Therefore both $y$ and $z$ unlock $x$ by compatibility, each alone in one of the sets $l(x), c(x)$, or $r(x)$ by Corollary 5.5. Switching $y$ and $z$ if necessary, as well as "left" and "right", we thus have $l(x)=\{y\}$ (hence $y<x$ by Lemma 5.9 (i)) and, either $c(x)=\{z\}$ or $r(x)=\{z\}$. (As we will see below, the first case is impossible.) Now the proof splits in two cases.

Case 1: $x \in \mathbf{L}$. If $l(x)=\{y\}$ and $c(x)=\{z\}$, then both $s$ and $t$ belong to $r(x)$. This implies that $y$ and $z$ are in $(x s)^{+}$, hence $x \neq x_{1}$ and $z \notin V\left(x_{2}\right)$, hence $z \notin \mathbf{T}$, a contradiction, see Figure 13 left.

As a consequence, one has, for all $P \in \mathcal{F}$ locking $x, l_{P}(x)=\{y\}, s, t \in c_{P}(x)$ and $r_{P}(x)=$ $\{z\}$. We fix some $P \in \mathcal{F}$ locking $x$.
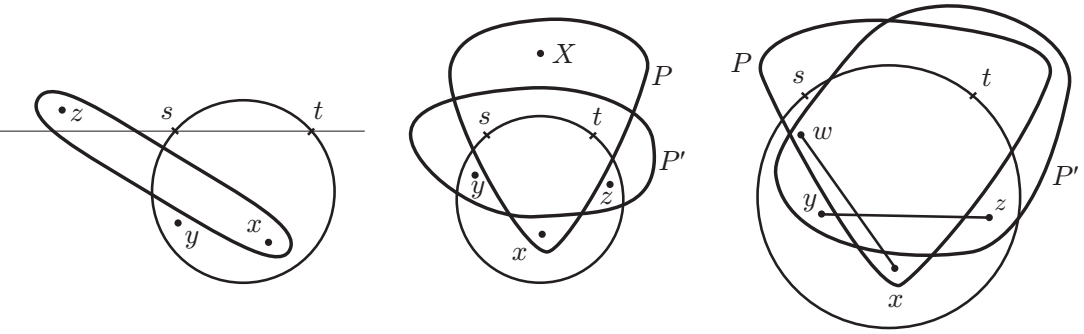

Figure 13: Proof of Theorem 5.10.

The key-idea is now to consider $D(x)$, which has to be nonempty by (11). Because from Lemma 5.9 (i) $y<x<z$ we have $x \neq x_{1}$ and $x \neq x_{m}$, hence $x$ cannot unlock by convexity. In other words, all points of $D(x)$ are visible.

Assume $D(x) \cap \mathbf{K} \neq \emptyset$ and let $X \in D(x) \cap \mathbf{K}$. If $X \in \mathbf{K} \backslash P$, then one easily checks that $X$ is already locked by $P$, with $s \in l_{P}(X), y, z \in c_{P}(X)$ and $t \in r_{P}(X)$, contradicting $x \rightarrow X$.

If $X \in P$ and $P^{\prime} \in \mathcal{F}$ is such that $x \rightarrow P_{P^{\prime}} X$, then $c_{P^{\prime}}(X)=\{x\}$, hence $y, z \in P^{\prime} \backslash P$. Because $x, X \in P \backslash P^{\prime}$ and $] x, X[\cap] y, z\left[\neq \emptyset, P\right.$ and $P^{\prime}$ would be incompatible, a contradiction, see Figure 13 middle.

Assume now that there exists $w \in D(x) \cap \mathbf{L}$. If $y<w<z$ then, because $l_{P}(x)=\{y\}$ and $r_{P}(x)=\{z\}$, we have $w \in P$. Actually, if $w \notin P$ then, in the case $y<w<x$ we have ]$w, z[\cap] x, X\left[\neq \emptyset\right.$ hence $w$ would be in $l_{P}(x)$, and in the case $x<w<z$ we would similarly have $w \in r_{P}(x)$. Moreover, since $s<y<w<z$, we have $] w, s[\cap] y, z[\neq \emptyset$, showing that $w$ is locked by $P$, with $y \in l_{P}(w), s \in c_{P}(w)$, and $z \in r_{P}(w)$, hence contradicting $x \rightarrow w$.

If $w \leq y$ and $P^{\prime} \in \mathcal{F}$ is such that $x \underset{P^{\prime}}{\rightarrow} w$ then, by Lemma $5.9, r_{P^{\prime}}(w)=\{x\}$. Since $y \in \overline{(w x)^{-}}$ and $y \notin r_{P^{\prime}}(w), y$ must be in $P^{\prime}$. We prove below by contradiction that $z$ itself is in $P^{\prime}$, see Figure 13 right.

Indeed, suppose that $z \notin P^{\prime}$. Since $z \notin r_{P^{\prime}}(w)$, we must have $z \in l_{P^{\prime}}(w)$. This implies $s, t \notin c_{P^{\prime}}(w) \subset(w x)^{+} \cap(w z)^{-}$, hence $s, t \in l_{P^{\prime}}(w)$ hence $s, t \notin P^{\prime}$. A point $X$ in $c_{P^{\prime}}(w)$ satisfies 
]$x, z[\cap] w, X\left[\neq \emptyset\right.$ hence $X \in(x z)^{-}$, thus $X$ cannot belong to $c_{P}(x)$. Therefore $X \notin P$. Now two cases occur.

If $w=y$ then $w \in P^{\prime} \backslash P$. This gives $w, X \in P^{\prime} \backslash P, s, x \in P \backslash P^{\prime}$, and $] w, X[\cap] s, x[\neq \emptyset$, thus $P, P^{\prime}$ incompatible, a contradiction.

If $w<y$, the points $s$ and $x$ are not in the triangle $\operatorname{conv}(\{w, y, X\})$ because its vertices are in $P^{\prime}$. As a consequence, the segment $] s, x[$, which cuts the side $w X$ of the triangle $\operatorname{conv}(\{w, y, X\})$ must cut another side. Since $y<x$, this segment cannot cross $[w, y]$, therefore it has to cut ]$y, X\left[\right.$. To sum up, we have $s, x \in P \backslash P^{\prime}, y, X \in P^{\prime} \backslash P$, and $] y, X[\cap] s, x[\neq \emptyset$, contradicting the compatibility of $P$ and $P^{\prime}$. This proves that $z \in P^{\prime}$.

Again the compatibility of $P$ and $P^{\prime}$ together with $] s, x[\cap] y, z\left[\neq \emptyset\right.$ imply $s \in P^{\prime}$. It follows that $s \in c_{P^{\prime}}(w)$ and that a point $v \in l_{P^{\prime}}(w)$ is such that $s<v<w$. The inequalities $v<w \leq y<x<z$ now imply $] v, x[\cap] y, z\left[\neq \emptyset\right.$ and $l_{P}(x)=\{y\}$ implies $v \in P$, in contradiction with the compatibility of $P$ and $P^{\prime}$.

Case 2: $x \in \mathbf{K}$. This case is more tricky because, contrarily to $\mathbf{L}$, the points of $\mathbf{K}$ are not necessarily in convex position. Before the proof, we present several intermediate lemmas. In the sequel, given three non-collinear points $a, b, c$ of the plane, $C(a, b, c)$ denotes the open cone with vertex $b$ and with $a$ and $c$ on its boundary, i.e.

$$
C(a, b, c)=\{b+\alpha(a-b)+\beta(c-b) ; \alpha, \beta>0\} .
$$

If $c \in(b a)^{+}$, then $C(a, b, c)=(b a)^{+} \cap(b c)^{-}$, otherwise $C(a, b, c)=(b c)^{+} \cap(b a)^{-}$.

Lemma 5.11. Let $X$ be a visible point in $\mathbf{K}$. Suppose that $X$ is locked by a set $P \in \mathcal{F}$ containing neither $s$ nor $t$. Then $X$ cannot unlock a point of $\mathbf{L}$.

Proof. Since $X$ is visible, $\mathbf{L}$ is included in the cone $C(s, X, t)$. By contradiction, suppose that $X \underset{P^{\prime}}{\rightarrow} w \in \mathbf{L}$. Let $Y$ be in $l_{P}(X)$ and $Z$ be in $r_{P}(X)$. Since $P$ contains neither $s$ nor $t, s$ and $t$ are in $c_{P}(X)$, hence $] Y, Z[$ cuts $] X, s[$ and $] X, t\left[\right.$ in two points $Y^{\prime}$ and $Z^{\prime}$. Since $X \underset{P^{\prime}}{\rightarrow} w$, $c_{P^{\prime}}(w)=\{X\}$, hence $Y, Z \notin P^{\prime}$. The segment $] X, w[$ cuts the segment $] Y^{\prime}, Z^{\prime}$ [ (hence the segment $] Y, Z[)$ in a point $p$ which is in $(s t)^{+}$. Since $P$ and $P^{\prime}$ are compatible, the point $w$ must be in $P$. Now the segment $] w, p$ [is in $\operatorname{conv}(Y, Z, w) \subseteq \operatorname{conv}\left(P \backslash\left(P_{0} \backslash\{w\}\right)\right)$ and cuts the segment ]$s, t\left[\subseteq \operatorname{conv}\left(\widetilde{P_{0}} \backslash P\right)(\right.$ at the same point as $] w, X[)$, i.e. we have $\operatorname{conv}\left(P \backslash\left(P_{0} \backslash\{w\}\right)\right) \cap \operatorname{conv}\left(\widetilde{P_{0}} \backslash P\right) \neq$ $\emptyset$. This is exactly the definition of $P$ locks $w$. Since $X \notin P$, removing $X$ would not unlock $w$, in contradiction with $X \rightarrow w$.

Lemma 5.12. Let $P, P^{\prime}$ be in $\mathcal{F}$ and $U, W$ be in $\mathbf{K}$. Suppose that $s \notin P, P$ locks $W$, and $W \underset{P^{\prime}}{\rightarrow} U$. Then $s \notin P^{\prime}$.

In particular, if $V \underset{P}{\rightarrow} W \underset{P^{\prime}}{\rightarrow} U$ and $s \notin P$, then $s \notin P^{\prime}$.

The same is true with $t$ instead of $s$.

Proof. We focuse on $s$. Observe first that $s \in c_{P}(W)$. Observe also that, because $W \in \mathbf{K}$ unlocks $U, U$ is visible. We act by contradiction and suppose that $s \in P^{\prime}$. We assume that $U<W$; The case $W<U$ is similar. By Lemma $5.9, r_{P^{\prime}}(U)=\{W\}$, hence $s \in l_{P^{\prime}}(U)$. Choose the point $V$ in $l_{P}(W)$ such that the open cone $C(V, s, W)$ contains no point of $l_{P}(W)$; It is possible to have $V=U$. We also choose an arbitrary point $X$ in $r_{P}(W)$ and an arbitrary point $u$ in $c_{P^{\prime}}(U)$. We have $V \in l_{P}(W), s \in c_{P}(W)$, and $X \in r_{P}(W)$, hence $] V, X[\cap] s, W[\neq \emptyset$. Since $s, W \in P^{\prime} \backslash P$, by compatibility of $P$ and $P^{\prime}$, one at least of the two points $V$ or $X$ must be in $P^{\prime}$. The proof now splits in four cases.

Case 1. $V \notin P^{\prime}$ (hence $X \in P^{\prime}$ ) and $u \in C(W, s, X)$.

The point $u$ is not in $P^{\prime}$, hence $u$ is not in the triangle $\operatorname{conv}(\{W, s, X\})$. Therefore, each segment ]$s, u[$ and $] V, X[$ starts from a different vertex of the triangle $s W X$ and cuts the corresponding 
opposite edge, hence these segments cross. The compatibility of $P$ and $\left(P_{0},\{s, t\}\right)$ implies that $u \in P$. Now we have

$$
W \notin P \Rightarrow W \notin \operatorname{conv}(\{V, X, u\}) \Rightarrow] V, u[\cap] s, W[\neq \emptyset,
$$

which contradicts $P$ and $P^{\prime}$ compatible.

Case 2. $V \notin P^{\prime}$ (hence $\left.X \in l_{P^{\prime}}(U)\right)$ and $u \notin C(W, s, X)$.

The segment $] U, u$ [ starting from $U$ must enter $C(W, s, X)$ by a point of the segment $] s, W$ [ then goes through the triangle $\operatorname{conv}(\{W, s, X\})$, leaves this triangle crossing the segment $] W, X$ [ and then leaves $C(W, s, X)$ crossing the line $(s X)$ at a point $a$ which is not in $[s, X]$ and then arrives at $u$. Therefore $X \in[s, a]$ and $a \in[U, u]$, yielding $X \in C(U)$ and contradicting $U$ visible.

Case 3. $V \in P^{\prime}$ and $U \in C(W, s, V)$.

We have $s, V, W \in P^{\prime}$ and $U \notin P^{\prime}$, hence $U \notin \operatorname{conv}(\{W, s, V\})$.

Since $r_{P^{\prime}}(U)=\{W\}$ we have $V \in l_{P^{\prime}}(U)$, hence $U \notin C(s, W, V)$. By the choice of $V, U$ is not in $P$. Moreover $U \notin P^{\prime}$, hence $U \notin \operatorname{conv}(\{W, s, V\})$. As in step 1, each of the segments ] $s, U[$ and $] V, X$ [ starts from a different vertex of the triangle $s V W$ and cuts the opposite edge, hence they cross. Since $s, U \notin P$ and $V, X \in P$, Remark 5.2 implies that $P$ locks $U$, which contradicts $W \rightarrow U$.

Case 4. $V \in P^{\prime}$ and $U \notin C(W, s, V)$.

The segment ] $u, U$ [ enters the cone $C(W, s, V)$ by a point of the segment ]s, $W[$, then goes through the triangle $\operatorname{conv}(\{W, s, V\})$, leaves this triangle crossing the segment $] W, V$ [, then leaves $C(W, s, V)$ crossing the line $(s V)$ at a point $b$ which is not in $[s, V]$, and then arrives at $U$. Therefore $V \in[s, b]$ and $b \in[U, u]$, which contradicts $U$ visible.

Lemma 5.13. Let $U, V, W \in \mathbf{K}$ be such that $V<W$ and assume $P \in \mathcal{F}$ locks $W$. We assume $s \notin P, l_{P}(W)=\{V\}$, and $W \rightarrow U$. Then either $U<V$ or $U>W$.

In particular, if $V<W, V \rightarrow \underset{P}{\rightarrow} W \rightarrow U$, and $s \notin P$, then either $U<V$ or $U>W$.

The same holds with $t$ in place of $s$.

Proof. Suppose on the contrary that $V \leq U<W$. Let $P^{\prime} \in \mathcal{F}$ locking $U$. By Lemma 5.9, $r_{P^{\prime}}(U)=\{W\}$. Moreover, by Lemma 5.12, $s \notin P^{\prime}$, thus $s \in c_{P^{\prime}}(U)$. Therefore $l_{P^{\prime}}(U) \subset$ $(s W)^{+} \cap(s U)^{+}$. Choose a point $X$ in $r_{P}(W)$ and a point $Y$ in $l_{P^{\prime}}(U)$.

If $U=V$, then $U$ is the only point in $l_{P}(W)$. The point $Y$ which is on the left side of $(s W)$, cannot be in $P$, otherwise it would be in $l_{P}(W)$. Likewise, $X$ cannot belong to $P^{\prime}$ for $W$ is the only point in $r_{P^{\prime}}(U)$. Starting at $Y$, the segment $] Y, W$ [ cuts the segment ],$U$ [ and must leave the triangle $\operatorname{conv}(\{V, s, X\})$, thus $] V, X[\cap] W, Y[\neq \emptyset$, but this contradicts the compatibility of $P$ and $P^{\prime}$. So it remains to show that $V<U<W$ is impossible. Observe that $U$ is not in $P$ for $s \in c_{P}(W)$ and $l_{P}(W)=\{V\}$. Two cases occur.

If $U \in(V X)^{+}$, then Remark 5.2 implies that $P$ locks $U$, hence $W$ cannot unlock $U$.

If $U \in(V X)^{-}$, then, since $s \in c_{P^{\prime}}(U)$, any $Y \in l_{P^{\prime}}(U)$ is in $C(U, W, s)$ but not in the triangle $\operatorname{conv}(\{U, W, s\})$. Since $U$ is in $(V X)^{-}$, the segment $] s, U[$ is included in the triangle $\operatorname{conv}(\{s, V, X\})$. Therefore the segment $] W, Y$ [, which cuts the segment $] s, U[$, must also cut ]$V, X$. Now $l_{P}(W)=\{V\}$ and $r_{P^{\prime}}(U)=\{W\}$ hence $Y \notin P$ and $X \notin P^{\prime}$. Moreover, since ]s, $U$ [ cuts the segment $] Y, W\left[, U\right.$ is in the triangle $\operatorname{conv}(\{Y, W, V\})$; Therefore if $V$ were in $P^{\prime}$ then $U$ would be in $P^{\prime}$. This proves that $V$ is not in $P^{\prime}$. To sum up, we have $V, X \in P \backslash P^{\prime}$, $W, Y \in P^{\prime} \backslash P$, and $] V, X[\cap] W, Y\left[\neq \emptyset\right.$, hence $P$ and $P^{\prime}$ incompatible, a contradiction.

Lemma 5.14. Let $k \geq 2$ and let $X_{1}<X_{2}<\ldots<X_{k}$ and $X_{k+1}$ be in $\mathbf{K}$. Suppose that

$$
X_{1} \underset{P_{1}}{\rightarrow} X_{2} \underset{P_{2}}{\rightarrow} \ldots X_{k-1} \underset{P_{k-1}}{\rightarrow} X_{k} \underset{P_{k}}{\rightarrow} X_{k+1}
$$

and that $s \notin P_{1}$. Then either $X_{k+1}<X_{1}$ or $X_{k+1}>X_{k}$. 
Proof. By Lemma 5.12, we have $s \notin P_{i}$ for all $i=1, \ldots, k$. Suppose on the contrary that $X_{1} \leq X_{k+1}<X_{k}$. Without loss of generality we can suppose that $X_{1} \leq X_{k+1}<X_{2}$. By Lemma 5.13, the integer $k$ must be at least 3 .

For each $i<k$, choose $X_{i}^{\prime} \in r_{P_{i}}\left(X_{i+1}\right)$ and let $Y_{i}$ denote the intersection point of the segments $] s, X_{i+1}[$ and $] X_{i}, X_{i}^{\prime}[$, see Figure 14 . Let $\mathcal{L}$ denote the polygonal line

$$
\mathcal{L}=\left[X_{1}, Y_{1}\right] \cup\left[Y_{1}, X_{2}\right] \cup\left[X_{2}, Y_{2}\right] \ldots \cup\left[X_{k-1}, Y_{k-1}\right] .
$$

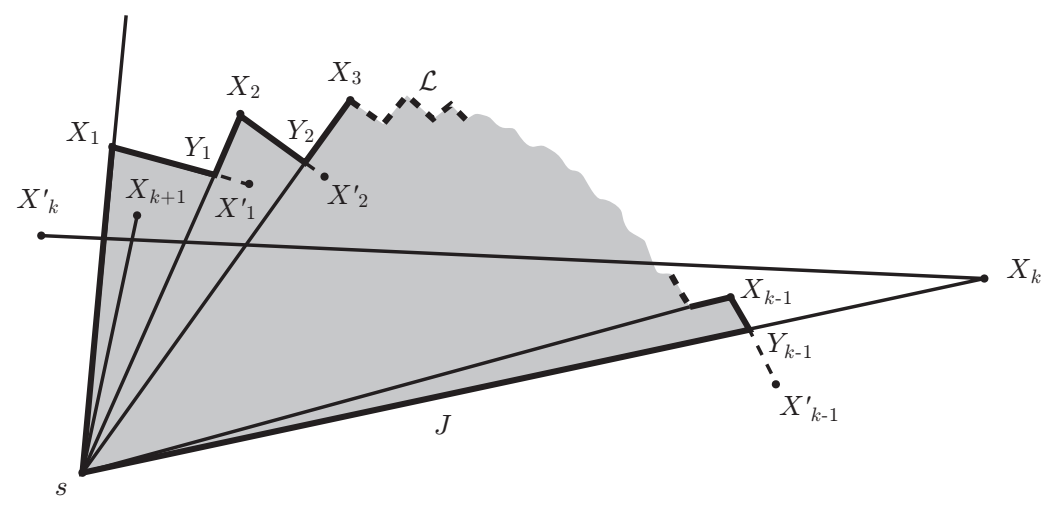

Figure 14: Proof of Lemma 5.14.

By definition of the order on $\mathbf{K}$, the line $\mathcal{L}$ is simple. Finally choose $X_{k}^{\prime} \in l_{P_{k}}\left(X_{k+1}\right)$.

If $r_{P_{1}}\left(X_{2}\right)=\left\{X_{k}\right\}\left(=\left\{X_{1}^{\prime}\right\}\right)$ then by Lemma 5.13, $X_{2}$ cannot unlock a point $X$ such that $X_{2}<X \leq X_{k}$ but this contradicts $X_{2} \underset{P_{2}}{\rightarrow} X_{3}$ with $X_{2}<X_{3} \leq X_{k}$. So we can suppose that $X_{1}^{\prime} \neq X_{k}$.

If $X_{k+1}$ were in the half plane $\left(X_{1} X_{1}^{\prime}\right)^{+}$, it would be locked by $P_{1}$ and not unlocked by $X_{k}$. Therefore $X_{k+1}$ is either in the interior of the triangle $\operatorname{conv}\left(\left\{s, X_{1}, Y_{1}\right)\right\}$ or is equal to $X_{1}$.

In the first case, $X_{k+1}$ is in the bounded region delimited by the Jordan curve $J=\left[s, X_{1}\right] \cup$ $\mathcal{L} \cup\left[Y_{k-1}, s\right]$ while $X_{k}$ is in the unbounded region. It follows that, starting at $X_{k}$, the segment $\left[X_{k}, X_{k}^{\prime}\right]$ must cross a segment $\left[X_{i}, Y_{i}\right]$ of the line $\mathcal{L}$. If $X_{k+1}=X_{1}$, the segment $\left[X_{k}, X_{k}^{\prime}\right]$ crosses ]$s, X_{1}$ [ and hence enters the bounded region of $J$. So the same result holds. In either case, choose $i$ maximal $(i \leq k-1)$ with $\left[X_{k}, X_{k}^{\prime}\right] \cap\left[X_{i}, Y_{i}\right] \neq \emptyset$.

The point $X_{k}^{\prime}$ is in the half plane $\left(s X_{i+1}\right)^{+}$, therefore, if $X_{k}^{\prime}$ were in $P_{i}$, it would be in $l_{P_{i}}\left(X_{i+1}\right)$ which is reduced to $X_{i}$. Moreover $X_{k}^{\prime}$ is in $\left(s X_{2}\right)^{+}$, hence this implies $X_{k}^{\prime}=X_{1}=X_{i}$. However this is impossible because the segment $\left[X_{k}, X_{k}^{\prime}\right]$ would not have met the bounded region delimited by the Jordan curve $J$. Therefore $X_{k}^{\prime} \notin P_{i}$.

We now prove that $X_{k} \notin P_{i}$. Indeed, the triangle $\operatorname{conv}\left(\left\{X_{k}, X_{i}, Y_{i}\right\}\right)$ contains the point $X_{i+1}$ because, viewed from $s$, the segment $] X_{k}, X_{i}$ [ is behind the segment ] $X_{k}^{\prime}, X_{k}$ [, which in turn is behind $X_{i+1}$, while the segment $] Y_{i}, X_{k}$ [ is between $s$ and $X_{i+1}$. Therefore $X_{k} \in P_{i}$ would imply $X_{i+1} \in P_{i}$, which contradicts $P_{i}$ locks $X_{i+1}$.

So we have proved that neither $X_{k}$ nor $X_{k}^{\prime}$ are in $P_{i}$. If $i \geq 2$, then $X_{i}$ and $X_{i}^{\prime}$ are in $\left(s X_{k+1}\right)^{+}$, hence they are not in $P_{k}$. If $i=1$, the segment ] $X_{1}, X_{k}$ [ does not cross the segment ] $s, X_{k+1}$ [ hence $X_{1} \notin l_{P_{k}}\left(X_{k+1}\right)$ and therefore $X_{1} \notin P_{k}$. Furthermore, the point $X_{1}^{\prime}$ is in $\left(s X_{k+1}\right)^{+}$and is $\neq X_{k}$ hence it cannot be in $P_{k}$. It follows that $P_{k}$ and $P_{i}$ are incompatible.

Continuation of proof of Theorem 5.10. Suppose that $y$ and $z$ in $\mathbf{T}$ unlock $x$ in $\mathbf{K}$. As before we prove first that $s$ and $t$ belong to $c(x)$. Otherwise we would have, say $l(x)=\{y\}, c(x)=\{z\}$, and $s, t \in r(x)$, hence $x$ would be hidden, a contradiction with $y \rightarrow x$. It follows that $y$ and $z$ are in $\mathbf{K}$. Set $X_{1}=x$. The point $X_{1}$ must unlock a point $X_{2}$ which, by Lemma 5.11 is in $\mathbf{K}$, and by Lemma $5.12, s, t \in c\left(X_{2}\right)$. By the same arguments, $X_{2}$ unlocks a point $X_{3}$ in $\mathbf{K}$ with 
$s, t \in c\left(X_{3}\right)$, and so on. We obtain an infinite sequence $X_{1}=x \underset{P_{1}}{\rightarrow} X_{2} \underset{P_{2}}{\rightarrow} \ldots X_{n-1} \underset{P_{n-1}}{\rightarrow} X_{n} \rightarrow \ldots$ of points in $\mathbf{K}$ such that $s, t \in c\left(X_{n}\right)$ for all $n$.

Now, we use the fact that $\mathbf{K}$ is a a subset of the finite set $S$. Consider $j \in \mathbb{N}$ such that $X_{j}=$ $\min \left(X_{n}\right)_{n \in \mathbb{N}}$ (for the order in $\mathbf{K}$ ). By Lemma 5.14 the sequence $\left(X_{j}, X_{j+1}, \ldots\right)$ is increasing, hence injective, a contradiction with $\mathbf{K}$ finite. This ends the proof of Theorem 5.10.

Corollary 5.15. In restriction to $\mathbf{T}$, the unlocking relation yields a bijection from $\mathbf{T}$ to $\mathbf{T}$.

Proof. By Theorem 5.10, all the sets $D(y), y \in \mathbf{T}$ are disjoint, yet they are non-empty subsets of $\mathbf{T}$ by (14), hence

$$
|\mathbf{T}| \leq \sum_{y \in \mathbf{T}}|D(y)|=\left|\bigcup_{y \in \mathbf{T}} D(y)\right| \leq|\mathbf{T}|,
$$

hence inequalities are equalities. The first equality gives $|D(y)|=1$ for all $y \in \mathbf{T}$, i.e. a point of $\mathbf{T}$ unlocks a unique point of $\mathbf{T}$, and the last one yields $\mathbf{T}=\bigcup_{y \in \mathbf{T}} D(y)$, i.e. each point of $\mathbf{T}$ is unlockable by a point of $\mathbf{T}$.

\subsection{Convexity of $\mathrm{K}$}

In this subsection, we will give an analog of Proposition 5.7 (i), but whose proof needs Corollary 5.15 . We begin by a statement which will be used several times, not only in this subsection but also in Subsection 5.9.

Lemma 5.16. (i) Let $A \neq B \in \mathbf{K}, W \in S \backslash\left(\widetilde{P_{0}} \cup\{A, B\}\right)$, and $y \in \widetilde{P_{0}}$. If $W \in \operatorname{conv}(y, A, B)$ and if $W$ is hidden by a point of $S \backslash \widetilde{P_{0}}$, then $C(W)$ contains a point $V \in \operatorname{conv}(y, A, B) \cap(S \backslash$ $\left.\left(\widetilde{P_{0}} \cup\{A, B\}\right)\right)$ with $C(V) \cap S=\emptyset$.

(ii) If $A<B$ are consecutive for the order on $\mathbf{K}$ then, for any $y \in \widetilde{P_{0}}, \operatorname{conv}(y, A, B)$ contains no point of $S \backslash\left(\widetilde{P_{0}} \cup\{A, B\}\right)$.

(iii) If $X<Y<Z$ are consecutive in $\mathbf{K}$ then, for every $y \in \widetilde{P_{0}}$ and every $W \in \operatorname{conv}(y, X, Z) \cap$ $\left(S \backslash\left(\widetilde{P_{0}} \cup\{X, Y, Z\}\right)\right)$, we have $Y \in C(W)$. Moreover, such a point $W$, if exists, has to be in $\operatorname{conv}(X, Y, Z)$.

Proof. (i) Recall that, if $U, V \in S \backslash \widetilde{P_{0}}$ are such that $U \in C(V)$, then $C(U) \subset C(V)$. As a consequence, since $S$ is finite, there exists $V \in C(W) \cap S$ such that $C(V) \cap S=\emptyset$. There exists $u \in \operatorname{relint}\left(\operatorname{conv}\left(\widetilde{P_{0}}\right)\right)$ such that $\left.V \in\right] u, W$. If $A=V$, then we would have $A$ and $W$ in $\operatorname{conv}(u, y, B)$, hence $B$ hidden, a contradiction. Similarly, we have $V \neq B$. Since $W \in$ $\operatorname{conv}(y, A, B)$, we obtain $V \in \operatorname{conv}(u, y, A, B)$. Since, by (15), $V$ is neither in $\operatorname{conv}(u, y, A)$, nor in $\operatorname{conv}(u, y, B)$, we must have $V \in \operatorname{conv}(y, A, B)$.

(ii) If $\operatorname{conv}(y, A, B)$ contained a point $W \in S \backslash \widetilde{P_{0}}$ then, by (i), $\overline{C(W)}$ would contain a point $V \in \operatorname{conv}(y, A, B) \cap\left(S \backslash\left(\widetilde{P_{0}} \cup\{A, B\}\right)\right)$ with $C(V) \cap S=\emptyset$. This point $V$ is in $\operatorname{conv}(s, y, A, B)$ but neither in $\operatorname{conv}(s, y, A)$, nor in $\operatorname{conv}(s, y, B)$, hence $V \in \operatorname{conv}(s, A, B)$, hence $V \in \mathbf{K}$ and $A<V<B$. This contradicts $A$ and $B$ consecutive.

(iii) If $C(W)=\emptyset$, set $V=W$, otherwise, let $V$ be given by (i). The point $V$ is in $\operatorname{conv}(s, y, X, Z)$ but neither in $\operatorname{conv}(s, y, X)$, nor in $\operatorname{conv}(s, y, Z)$, hence $V \in \operatorname{conv}(s, X, Z)$, hence $V \in K$ and $X<V<Z$, hence necessarily $V=Y$.

Moreover $W \in \operatorname{conv}(s, y, X, Y, Z)=\bigcup_{u, v \in\{s, y, X, Z\}} \operatorname{conv}(u, v, Y)$. By (ii), $W$ cannot be in $\operatorname{conv}(s, X, Y) \cup \operatorname{conv}(s, Y, Z) \cup \operatorname{conv}(y, X, Y) \cup \operatorname{conv}(y, Y, Z)$ and, since $C(Y) \cap S=\emptyset, W \notin$ $\operatorname{conv}(s, y, Y)$. Therefore $W \in \operatorname{conv}(X, Y, Z)$.

We now give the main statement of this subsection. 
Proposition 5.17. The points of $\mathbf{K}$ are in convex position. More precisely, for all $X<Y<$ $Z \in \mathbf{K}$, we have $Y \in(X Z)^{+}$.

Proof. It suffices to prove the statement for consecutive points.

First assume that $\widetilde{P_{0}} \cap(X Z)^{+} \neq \emptyset$ and consider $x \in \widetilde{P_{0}} \cap(X Z)^{+}$. Since $s \in(X Z)^{-}$, by (15) $x$ is neither in $(s X)^{+}$(otherwise $X$ would be in the triangle $\operatorname{conv}(Z, s, x)$ hence would hide $Z$ ) nor in $(s Z)^{-}(Z$ would hide $X)$, hence $x$ is in the cone $C(X, s, Z)$. From (15), we deduce that $Y \notin \operatorname{conv}(X, s, x) \cup \operatorname{conv}(s, x, Z)$, hence $Y \in C(X, s, Z) \backslash \operatorname{conv}(s, X, Z) \subset(X Z)^{+}$.

We now assume, until the end of this subsection, that $\widetilde{P_{0}} \cap(X Z)^{+}=\emptyset$. We proceed by contradiction, i.e. we assume that $Y \in(X Z)^{-}$. Therefore in the sequel we assume $\widetilde{P}_{0} \cup\{Y\} \subset$ $(X Z)^{-}$. Since $s \in(X Y)^{-} \cap(Y Z)^{-}$, by $(15)$ we have $\widetilde{P_{0}} \subset(X Y)^{-} \cap(Y Z)^{-}$. In particular, we have $Y \in \operatorname{conv}(X, x, Z)$ for all $x \in \widetilde{P_{0}}$. Observe also that, for all $x \in \widetilde{P_{0}}$, we have $X \in(x Y)^{+}$ and $Z \in(x Y)^{-}$. As a consequence, if $P \in \mathcal{F}$ locks $Y$ and $X \in P$, then necessarily $X \in l_{P}(Y)$.

Before going further, we would like to make the following remark. If some $W \in \mathbf{K}$ is hidden by $x_{1}$ (the first point of $\mathbf{L}$ ) then only $x_{1}$ can unlock $W$. From Corollary 5.15, it follows that $x_{1}$ can hide at most one point of $\mathbf{K}$, which must be the first point of $\mathbf{K}$. It is the same for $x_{m}$ if $m \geq 2$. As a consequence, $X$ may or may not be hidden by $x_{1}$, and $Z$ by $x_{m}$, but $Y$ cannot be hidden.

Lemma 5.18. For every $P \in \mathcal{F}$ locking $Y, P$ locks either $X$ or $Z$, or both.

Proof. Consider such a $P$ and let $y \in c_{P}(Y)$. Observe that, since at least one point $a$ of $] y, Y$ [is in $\operatorname{conv}(P)$ and since $Y \in \operatorname{conv}(a, X, Z) \backslash P$, the points $X$ and $Z$ cannot both be in $P$ : We must have either $X \notin P$ or $Z \notin P$ (or both). We divide the proof in several cases which possibly overlap, but exhaust all possible cases. We will see that each case ends either by a contradiction, or by " $P$ locks $X$ ", or by " $P$ locks $Z$ ".

Case 1. The sets $l_{P}(Y) \backslash\{s, t\}$ and $r_{P}(Y) \backslash\{s, t\}$ are both nonempty. Choose $U \in l_{P}(Y) \backslash$ $\{s, t\}$ and $V \in r_{P}(Y) \backslash\{s, t\}$, hence $] U, V[$ cuts $] y, Y[$.

If $U=X$, then $X \in P$, hence $Z \notin P$. Moreover $V \in C(y, X, Y) \cap(y Y)^{-}$, hence $V \notin$ $\operatorname{conv}(X, Y, Z)$, hence, by Lemma 5.16 (iii), $V \notin \operatorname{conv}(X, y, Z)$, hence $] X, V$ [ cuts ] $y, Z[$, hence $P$ locks $Z$. The case $V=Z$ is similar and yields that $P$ locks $X$.

We now assume that $U \neq X$ and $V \neq Z$. By Lemma 5.16 (iii), if $U \in \operatorname{conv}(y, X, Z)$ then $U \in \operatorname{conv}(X, Y, Z)$, idem for $V$, and if $U$ and $V$ are both in $\operatorname{conv}(X, Y, Z)$ then $] U, V$ [ cannot cross ] $y, Y$ [, hence $U$ and $V$ cannot both be in $\operatorname{conv}(y, X, Z)$. As a consequence, the open segment $] U, V[$ crosses one or both of the sides $] y, X[$ and $] y, Z[$ of the triangle $\operatorname{conv}(y, X, Z)$. We now split the proof into two subcases.

Subcase 1.1. The segment $] U, V[$ crosses both sides $] y, X[$ and $] y, Z[$ of $\operatorname{conv}(y, X, Z)$. As already said, one of the points $X$ or $Z$, say $X$, does not belong to $P$, and we obtain that $P$ locks $X$.

Subcase 1.2. The segment $] U, V[$ crosses one side, say $] y, X[$, and not $] y, Z[$. Then, by Lemma 5.16 (iii), $V \notin \operatorname{conv}(y, Y, Z)$, hence $V \in C(Y, U, Z) \cap(Y Z)^{+}$, hence $Y \in \operatorname{conv}(U, V, X)$, hence $X \notin P$, hence $P$ locks $X$.

Case 2. $l_{P}(Y)=\{s\}$ and $r_{P}(Y) \neq\{t\}$. Choose $V \in r_{P}(Y) \backslash\{t\}$.

Subcase 2.1. ],$V[$ crosses both ] $X[$ and $] y, Z[$. As in Subcase 1.1, the point among $X$ and $Z$ which is not in $P$ is locked by $P$.

Subcase 2.2. ] $s, V[$ crosses ] $y, X$ and not $] y, Z\left[\right.$. As in Subcase 1.2, $V \in C(Y, s, Z) \cap(Y Z)^{+}$, hence $Y \in \operatorname{conv}(s, V, X)$, hence $X \notin P$, hence $P$ locks $X$.

Subcase 2.3. ] $s, V[$ does not cross $] y, X[$. Then $s \in \operatorname{conv}(y, X, Z)$, hence $X$ is hidden by $y$, hence $y=x_{1}$ and is the unlocker of $X$ in $\mathbf{T}$. 
By Corollary 5.15, $y$ cannot be the unlocker of $Y$. Since $s \in l(Y)$ and $y \in c(Y)$, the unlocker of $Y$ must be $V$, with $\{V\}=r(Y)$, hence $V \in \mathbf{K}$ and $V \geq Z$.

If $V \neq Z$, then $Z \notin P$ (otherwise $Z \in r_{P}(Y)=\{V\}$ ), and ]s, $V$ [ and ] $y, Z[$ cross, hence $P$ locks $Z$.

If $V=Z$, i.e. $Z \rightarrow Y$, then any $Q \in \mathcal{F}$ locking $Y$ contains $Z$ (as right locker), hence does not contain $X$. Since $y$ is not the unlocker of $Y$, there exists at least one element $Q \in \mathcal{F}$ locking $Y$ such that $c_{Q}(Y) \neq\{y\}$. Once again, we split the proof in two cases.

If $s \in Q$, i.e. $s \in l_{Q}(Y)$, let $x \in c_{Q}(Y) \backslash\{y\}$. Then $x \in(s X)^{-}$(otherwise $x$ would hide $X$, contradicting $y \rightarrow X)$, hence, since $x \in P_{0} \subset(X Z)^{-}$, we have $x \in C(s, X, Z)$. Since $x \in(s Z)^{-}$, ]$x, X[$ and $] s, Z\left[\right.$ cross, with $s, Z \in Q \backslash P_{0}, x \in \widetilde{P_{0}} \backslash Q$, and $X \notin Q$. Therefore, if we remove $y$, $Q$ would still lock $X$, a contradiction with $y \rightarrow X$.

If $s \notin Q$, i.e. $s \in c_{Q}(Y)$, let $U \in l_{Q}(Y)$. Since $] U, Z[$ and $] s, Y$ [ cross, we have $U \in$ $C(s, Z, Y) \cap(s Y)^{+}$hence $U \neq t$ (because $\left.t \in(s Y)^{-}\right)$and $U \notin \operatorname{conv}(X, Y, Z)$, hence, by Lemma 5.16 (iii), $U \notin \operatorname{conv}(y, X, Z)$, hence $U \notin \operatorname{conv}(s, X, Z)$, hence $] s, X$ [ and $] U, Z[$ cross, once again showing that $Q$ locks $X$ and contradicting $y \rightarrow X$.

The case $l_{P}(Y) \neq\{s\}$ and $r_{P}(Y)=\{t\}$ is symmetrical.

Case 3. $l_{P}(Y)=\{s\}$ and $r_{P}(Y)=\{t\}$. Then the unlocker of $Y$ must be central, i.e. $y \rightarrow Y$. Among $X$ and $Z$, one at least is not in $P$, say $X \notin P$. If ] $s, t[$ and $] y, X$ [intersect, then $P$ locks $X$, hence the unlocker of $X$ must be $y$, a contradiction with $y \rightarrow Y$. If ] $s, t$ and ] $y, X$ [ do not intersect, then $y \in(s X)^{+}$, i.e. $y$ hides $X$, hence $y \rightarrow X$, contradicting $y \rightarrow Y$.

Case 4. $s \in r_{P}(Y)$, hence $t \in r_{P}(Y)$. Moreover, we have $y \in(s t)^{+}$(otherwise $y$ would hide $Y)$, hence $s \in(y Z)^{-}$. Let $U \in l_{P}(Y)$, then $] U, s[$ and $] y, Y$ [ cross, hence $U \in C(y, s, Y) \cap(y Y)^{+}$, hence $] U, s$ [ and ] $y, Z$ [ cross. If $Z \notin P$ then $P$ locks $Z$. Otherwise, we firstly have $X \notin P$ and secondly, since $U$ and $s$ are in $P, Y$ cannot be in the triangle $\operatorname{conv}(U, s, Z)$, hence $U \in(Y Z)^{-}$. It follows that $U \notin \operatorname{conv}(X, Y, Z)$, hence, by Lemma 5.16 (iii), $U \notin \operatorname{conv}(y, X, Z)$, hence $] U, s[$ and $] y, X[$ cross, hence $P$ locks $X$.

The last case $t \in l_{P}(Y)$ is symmetrical. This ends the proof of Lemma 5.18.

Continuation of proof of Proposition 5.17. Let $A \in \mathbf{T}$ be the unlocker of $Y$. If $A \in \mathbf{L}$ then consider any $P \in \mathcal{F}$ locking $Y$. By Lemma 5.18, $P$ locks either $X$ or $Z$ (or both), say $P$ locks $X$. We have $c_{P}(Y)=\{A\}, s \in l_{P}(Y)$, and $t \in r_{P}(Y)$.

If ] $A, X$ [ and ] $s, t$ cross, then $A$ is the only possible unlocker of $X$, contradicting $A \rightarrow Y$.

If $] A, X[$ and $] s, t$ [ do not cross, then $A \in(s X)^{+}$, i.e. $A$ hides $X$ and, once again only $A$ can unlock $X$, contradicting $A \rightarrow Y$.

It follows that $A$ is in $\mathbf{K}$. Without loss of generality, assume that $A<Y$, i.e. $\{A\}=l(Y)$. The proof splits in two cases.

Case 1. $A=X$. Consider any $P \in \mathcal{F}$ locking $Y$. We have $l_{P}(Y)=\{X\}$, hence $X \in P$, hence $P$ does not lock $X$. By Lemma 5.18, $P$ must lock $Z$.

Step 1: The point $Z$ is visible.

By contradiction, if $Z$ is hidden, then it is hidden by $x_{m}$, the last point of $\mathbf{L}$, hence $x_{m} \rightarrow Z$. Besides, since $Y$ is visible, we have $t \in C\left(Y, x_{m}, Z\right)$. Since $\widetilde{P_{0}} \subset(Y Z)^{-}$, this implies $t \in$ $\operatorname{conv}\left(x_{m}, Y, Z\right)$. Since $X, Z \in \mathbf{K} \subset(s t)^{+}$, we also have $s \in(t Z)^{+} \cap(X t)^{-}$, hence $s \in\left(x_{m} Z\right)^{+}$. If $s$ were in $\left(x_{m} X\right)^{-}$, then $X$ would be hidden by $x_{m}$, hence $x_{m}$ would be the only possible unlocker of $X$, contradicting $x_{m} \rightarrow Z$, therefore $s \in\left(x_{m} X\right)^{+} \cap\left(x_{m} Z\right)^{+}$.

Since $P$ locks $Z$, there exist $U, V \in P \backslash P_{0}$ and $x \in \widetilde{P_{0}} \backslash P$ such that $] x, Z$ [ and $] U, V$ [ cross. Without loss, we assume $U \in\left(x_{m} Z\right)^{+}$and $V \in\left(x_{m} Z\right)^{-}$(we cannot talk about $l_{P}(Z)$ and $r_{P}(Z)$ because these categories are defined for visible points only).

We necessarily have $x=x_{m}$, otherwise removing $x_{m}$ would not unlock $Z$, hence $x_{m} \notin P$. We have $x_{m} \in c_{P}(Y)$ and $s \in\left(x_{m} Y\right)^{+}$, hence $s \notin P$ (otherwise $s$ would be in $l_{P}(Y)=\{X\}$ ). Now the proof splits in two cases: $U=X$ or $U \neq X$. 
If $U=X$, then $V \in r_{P}(Y) \subset C\left(x_{m}, X, Y\right)$, hence $V \in C\left(x_{m}, X, Y\right) \cap\left(x_{m} Z\right)^{-}$. Since $] s, Y$ [ and $] X, V$ [ cross, this implies that ]s, $Z$ [ and $] X, V$ [ cross. To sum up, we have $X, V \in P \backslash P_{0}$, $s \in \widetilde{P_{0}} \backslash P, Z \notin \widetilde{P_{0}} \cup P$, and $] s, Z[\cap] X, V\left[\neq \emptyset\right.$, therefore, if we remove $x_{m}, Z$ is still locked by $P$, a contradiction with $x_{m} \rightarrow Z$.

If $U \neq X$, then $U \in r_{P}(Y) \subset\left(x_{m} Y\right)^{-} \cap C\left(x_{m}, X, Y\right)$. Since $U \in\left(x_{m} Z\right)^{+}$, this implies $U \in \operatorname{conv}\left(x_{m}, Y, Z\right)$, hence, by Lemma 5.16 (iii), $U=s$ or $t$, hence $U=t$ since $s \in\left(x_{m} Y\right)^{+}$. Now we have $X<Z$, hence $] s, Z[\cap] t, X\left[\neq \emptyset\right.$ with $t, X \in P \backslash P_{0}, s \in \widetilde{P_{0}} \backslash P$, and $Z \notin \widetilde{P_{0}} \cup P$, hence removing $x_{m}$ would not unlock $Z$.

If $U \in\left(x_{m} Y\right)^{+}$, then $U \in l_{P}(Y)$ hence $U=X$.

Step 2: $W$ e have $l_{P}(Y)=l_{P}(Z), c_{P}(Y)=c_{P}(Z)$, and $l_{P}(Y)=l_{P}(Z)$.

We have $c_{P}(Y)=\widetilde{P_{0}} \backslash P=c_{P}(Z)$. Since $Y<Z$ and $\widetilde{P_{0}} \subset(Y Z)^{-}$, we also have $l_{P}(Y) \subseteq$ $l_{P}(Z)$ and $r_{P}(Z) \subseteq r_{P}(Y)$. Actually, if $x$ is any point in $c_{P}(Y)$, then we have $l_{P}(Y) \cap r_{P}(Z)=$ $(x Y)^{+} \cap(x Z)^{-} \cap\left(P \backslash P_{0}\right)$. If this set is nonempty, consider $U \in l_{P}(Y) \cap r_{P}(Z)$ and $V \in r_{P}(Y)$, then $] U, V$ [ crosses ]x, $Y$ [ hence $V \in C(Y, x, Z)$, hence $V \in(x Z)^{+}$, hence $V \in l_{P}(Z)$, but $] U, V$ [ cannot cross $] x, Z[$, a contradiction.

Let us show by contradiction that $l_{P}(Z)=l_{P}(Y)$. Choose $y \in c_{P}(Y)$. A point $W \in$ $l_{P}(Z) \backslash l_{P}(Y)$ is in $l_{P}(Z) \cap r_{P}(Y)$, hence $W \in C(Y, y, Z)$. Moreover $] X, W$ [ crosses $] y, Y$ [, hence $W \in \operatorname{conv}(y, Y, Z) \cap S \backslash\left(\widetilde{P_{0}} \cup\{X, Y, Z\}\right)$. By Lemma 5.16 (iii), it follows that $W \in \widetilde{P_{0}}$, hence $W=s$ or $t$.

If $W=s$ then $t \in(X s)^{+} \cap(s Z)^{-}$, hence $y \in(s t)^{-}$, hence $y \in \mathbf{L}$, hence $y$ hides $Y$, hence $y \rightarrow Y$, contradicting $X \rightarrow Y$.

If $W=t$, then $y$ hides $Z$, contradicting $Z$ visible. This proves that $l_{P}(Z)=l_{P}(Y)$.

Because $l_{P}(Y)$ and $r_{P}(Y)$ form a partition of $P \backslash \widetilde{P_{0}}$, we deduce that $r_{P}(Z)=r_{P}(Y)$ as well.

Step 3: To sum up, we have $l_{P}(Z)=l_{P}(Y), c_{P}(Z)=c_{P}(Y)$, and $r_{P}(Z)=r_{P}(Y)$ for all $P \in \mathcal{F}$ that locks $Y$. As a consequence, we obtain $l(Y) \subseteq l(Z), r(Y) \subseteq r(Z)$, and $c(Y) \subseteq c(Z)$. This implies that any point unlocking $Z$ unlocks $Y$, a contradiction with Corollary 5.15.

Case 2. $A \neq X$. Then we have $A<X$ and, for all $P \in \mathcal{F}$ locking $Y$ we have $X \notin P$ (otherwise $X$ would be in $l_{P}(Y)=\{A\}$, a contradiction). Once again, we split the proof in two subcases.

Subcase 2.1. There exists $P \in \mathcal{F}$ locking $Y$ such that neither $s$ nor $t$ is in $P$. In that case, by Lemma 5.11, $Y$ can unlock only a point $Y_{1}$ in $\mathbf{K}$, with a subset $P_{1}$ containing neither $s$ nor $t$ by Lemma 5.12. In the same way we have, for any arbitrarily large $n \in \mathbb{N}, n$ points $Y_{i} \in \mathbf{K}$ and $n$ subsets $P_{i} \in \mathcal{F}$ such that $Y_{1} \underset{P_{2}}{\rightarrow} Y_{2} \underset{P_{2}}{\rightarrow} \cdots \underset{P_{n}}{\rightarrow} Y_{n}$, with $s, t \notin P_{i}$ for all $i \in\{1, \ldots, n\}$. Now Lemma 5.14 shows that all these points are different, a contradiction with $\mathbf{T}$ finite.

Subcase 2.2. For all $P \in \mathcal{F}$ locking $Y$ we have either $s \in P$ or $t \in P$ (or both). Observe that, for any $P \in \mathcal{F}$, if $s \in P$ then $s \in r_{P}(Y)$ (since $l_{P}(Y)=\{A\}$ ). Because $] A, s[\cap] t, Y[=\emptyset$, it follows that $t$ cannot belong to $c_{P}(Y)$, hence $t \in P$. As a consequence, in this case 2.2 we have in fact $t \in r_{P}(Y)$ for all $P \in \mathcal{F}$ locking $Y$.

Now, $c_{P}(Y) \subset C(A, Y, t) \cap(A t)^{-} \backslash(A X)^{+}$(otherwise $A$ would be in $\left.C(X)\right)$, hence any $P$ locking $Y$ locks $X$, with $c_{P}(Y)=c_{P}(X)\left(=\widetilde{P_{0}} \backslash P\right), l_{P}(X) \subseteq l_{P}(Y)$, and $r_{P}(Y) \subseteq r_{P}(X)$ (because $X<Y$ and $\left.\widetilde{P_{0}} \subset(X Y)^{-}\right)$. Nevertheless, we have $A \rightarrow Y$, hence $l_{P}(Y)=\{A\}$, and $l_{P}(X) \neq \emptyset$, therefore we obtain $l_{P}(X)=l_{P}(Y)$, hence also $r_{P}(X)=r_{P}(Y)$.

Since this is true for any $P \in \mathcal{F}$ locking $Y$, we obtain as before $l(Y) \subseteq l(X), r(Y) \subseteq r(X)$, and $c(Y) \subseteq c(X)$, hence any point unlocking $X$ unlocks $Y$, a contradiction with Corollary 5.15. 


\subsection{A first analysis of the orbits}

A priori we have little knowledge on the unlocking relation $\rightarrow$ on $S \backslash \mathbf{T}$, but we know that, in restriction to $\mathbf{T}$, it is bijective. In the sequel, we analyze the possible orbits of the map $\rightarrow$ in $\mathbf{T}$.

Lemma 5.19. (i) If $x, y \in \mathbf{L}$ are such that $y \rightarrow x$, then $x$ and $y$ are consecutive for the order on $\mathbf{L}$.

(ii) Similarly, if $X, Y \in \mathbf{K}$ are such that $Y \rightarrow X$, then $X$ and $Y$ are consecutive for the order on $\mathbf{K}$.

Proof. (i) Assume without loss that $x<y$. Consider $P \in \mathcal{F}$ that locks $x$. By Corollary 5.5, we have either $\{y\}=r_{P}(x)$ or $\{y\}=l_{P}(x)$ and by Lemma 5.9 (i), $\{y\}=r_{P}(x)$. Let $z$ denote the successor of $x$ for the order on $\mathbf{L}$. We have to show that $y=z$. By contradiction, assume that $y \neq z$; Then we have $z<y$, hence $z \in(x y)^{-}$. Since $\{y\}=r_{P}(x)$, the line below (12) implies $\left(\widetilde{P_{0}} \backslash P\right) \cap(x y)^{-}=\emptyset$, hence $z \in P$. A segment $] v, y\left[\right.$ with $v \in l_{P}(x)$ splits $\operatorname{conv}(P)$ into two connected components. The point $v$ is in $(x y)^{+}$but not in $(z y)^{-}$, otherwise $y$ would not be an extreme point of $\widetilde{P_{0}}$, contradicting Proposition 5.7 (i). It follows that the points $x$ and $z$ are both in $(v y)^{-}$, and therefore the connected component that contains $x$ also contains $z$. First, it follows that $P$ also locks $z$ and that $c_{P}(z)=c_{P}(x)$. Next, one has $v, y \in(x z)^{+}$and ]$x, U[\cap] v, y\left[\neq \emptyset\right.$ for all $U \in c_{P}(x)$, hence $c_{P}(x) \subset(x z)^{+}$. Since for any $U \in c_{P}(x)$ there is no point of $\widetilde{P_{0}} \backslash P$ in $(x U)^{-} \cap(z U)^{+}$, we have $l_{P}(z)=l_{P}(x)$ and $r_{P}(z)=r_{P}(x)$.

To sum up, we have $l_{P}(z)=l_{P}(x), c_{P}(z)=c_{P}(x)$, and $r_{P}(z)=r_{P}(x)$ for all $P \in \mathcal{F}$ that locks $x$. As a consequence, we obtain $l(x) \subseteq l(z), r(x) \subseteq r(z)$, and $c(x) \subseteq c(z)$. This implies that any point unlocking $z$ unlocks $x$, a contradiction with Corollary 5.15.

(ii) Without loss, assume that $X<Y$ and let $P$ be any element of $\mathcal{F}$ locking $X$. By Corollary 5.5 and Lemma 5.9 (ii), we have $\{Y\}=r_{P}(X)$. Let $Z$ denote the successor of $X$ for the order on $\mathbf{K}$. We have to prove that $Y=Z$. By contradiction, we assume $Y \neq Z$.

By Proposition 5.17, we have $Z \in(X Y)^{+}$, hence, by the very end of Proposition 5.3, $Z \notin P$ otherwise we would have $Z \in r_{P}(X)=\{Y\}$. We prove below that $P$ locks $Z$.

Case 1. $s \notin P$. Therefore $s \in c_{P}(X)$. Choose $V \in l_{P}(X)$. Since $V \in C(X, Y, s) \cap(s X)^{+}$and $Z \in C(X, s, Y)$, the segment $] V, Y$ [ cuts the segment ]s, $Z$ [, hence $P$ locks $Z$.

Case 2. $s \in P$. Since $r_{P}(X)=\{Y\}$, we then have $s \in l_{P}(X)$. Choose $u \in c_{P}(X)$. Since $] u, X[$ crosses ] $s, Y$ [, we have $u \in(s Y)^{-}$. Since $Z \in C(X, s, Y) \cap(X Y)^{+}$, and since $Y \notin C(s, Z, u)$, the segment ] $s, Y$ [ cuts the segment ]u,Z[, hence $P$ locks $Z$.

Now we conclude as in case (i): For all $P \in \mathcal{F}$ locking $X, P$ locks $Z$, with $l_{P}(Z)=$ $l_{P}(X), c_{P}(Z)=c_{P}(X)$, and $r_{P}(Z)=r_{P}(X)$. Indeed, for any $u \in c_{P}(X)$ we have $l_{P}(X) \subset$ $C(u, Y, X) \cap(u X)^{+} \subset(u Z)^{+}$, hence $l_{P}(X) \cap r_{P}(Z)=\emptyset$, hence $\emptyset \neq r_{P}(Z) \subseteq r_{P}(X)=\{Y\}$, hence $r_{P}(Z)=r_{P}(X)$, hence $l_{P}(Z)=l_{P}(X)$, too. It follows that $l(x) \subseteq l(z), r(x) \subseteq r(z)$, and $c(x) \subseteq c(z)$, therefore any point unlocking $Z$ unlocks $X$, a contradiction with Corollary 5.15.

Lemma 5.20. If $x, y \in \mathbf{T}$ are such that $y \rightarrow x$, then $x \not \rightarrow y$.

Proof. Assume by contradiction that $x \rightarrow y \rightarrow x$. Exchanging the roles of $x$ and $y$ if necessary, the proof splits in four cases, depending whether $x$ and $y$ are both in $\mathbf{L}$ or both in $\mathbf{K}$, or $x \in \mathbf{L}$ unlocks $y \in \mathbf{K}$ by compatibility or $x \in \mathbf{L}$ unlocks $y \in \mathbf{K}$ by convexity. For the first three cases, let $P, P^{\prime} \in \mathcal{F}$ be such that $x \underset{P}{\rightarrow} y \underset{P^{\prime}}{\rightarrow} x$.

Case 1. $x, y \in \mathbf{L}$. To fix ideas, we suppose $x<y$. By Lemma 5.9, we have $l_{P}(y)=\{x\}$ and $r_{P^{\prime}}(x)=\{y\}$.

Subcase 1.1. There exists $z \in\left(P \cap P^{\prime}\right) \backslash P_{0}=c_{P}(y) \cap c_{P^{\prime}}(x)$. Let $u \in r_{P}(y)$ and $v \in l_{P^{\prime}}(x)$. Since $] y, z\left[\subset(x z)^{-}\right.$and $] x, u[$ crosses $] y, z\left[, u\right.$ is in $(x z)^{-}$. It follows that $u \in P^{\prime}$, otherwise $u$ 
would be in $r_{P^{\prime}}(x)=\{y\}$. In the same way, we have $v \in P$. Now $] x, u[$ cuts $] y, v[$, a contradiction with $P$ and $P^{\prime}$ compatible.

Subcase 1.2. There exists $z \in P \backslash\left(P_{0} \cup P^{\prime}\right)=c_{P}(y) \backslash c_{P^{\prime}}(x)$. Let $u \in r_{P}(y)$. By compatibility of $P$ and $P^{\prime}$, $u$ cannot be in $P^{\prime}$, hence $u \in l_{P^{\prime}}(x)$. Let $w \in c_{P^{\prime}}(x)$. We have $w \in(y u)^{-}$and $u \in r_{P}(y)$, hence $w$ cannot be in $c_{P}(y)$, hence $w \notin P$. Now ] $x, w$ [ cuts $] y, z[$, a contradiction with $P$ and $P^{\prime}$ compatible.

Case 2. $x<y \in \mathbf{K}$. By Lemma 5.13, $s$ and $t$ are in $P \cap P^{\prime}$. This means that $s, t \in$ $l_{P^{\prime}}(x) \cap r_{P}(y)$. Let $u \in c_{P}(y)$ and $v \in c_{P^{\prime}}(x)$. Since $x \in(u y)^{+}$and $s \in(u y)^{-}$, the segment ] $u, x$ [ does not cross the segment ] $s, y$ [. It follows that $u \notin c_{P^{\prime}}(x)$, hence $u \in P^{\prime}$. The same way of reasonning gives $v \in P$. Since $] v, x[$ and $] u, y[$ cross opposite sides of the quadrilateral styx, these two segments cross, a contradiction with $P$ and $P^{\prime}$ compatible.

Case 3. $x \in \mathbf{L}$ unlocks $y \in \mathbf{K}$ by compatibility. We have $c_{P}(y)=\{x\}$, hence $s, t \in P$, and $c_{P^{\prime}}(x)=\{y\}$, hence $s, t \notin P^{\prime}$. Since $y$ is not locked by convexity, we also have $] s, t[\cap] x, y[\neq \emptyset$, a contradiction with $P$ and $P^{\prime}$ compatible.

Case 4. $x \in \mathbf{L}$ unlocks $y \in \mathbf{K}$ by convexity. In this last case, Remark 5.6 holds (with $x$ in the role of $y$ and $y$ in the role of $X$ ). Without loss of generality, we suppose that $x=x_{1}$ and $y \in V\left(x_{2}\right) \backslash V\left(x_{1}\right)$ (or simply $y \notin V\left(x_{1}\right)$ in the case $m=1$ ). Let $P^{\prime} \in \mathcal{F}$ be such that $y \underset{P^{\prime}}{\rightarrow}$. We obtain

$$
\emptyset \neq l_{P^{\prime}}(x) \subseteq(x y)^{+} \cap \widetilde{P_{0}} \subseteq(x s)^{+} \cap \widetilde{P_{0}}=\emptyset,
$$

a contradiction.

As a consequence of the two preceding lemmas, an orbit of $\rightarrow$ cannot stay always in $\mathbf{L}$ or always in $\mathbf{K}$, but has to pass alternatively from $\mathbf{L}$ to $\mathbf{K}$ and from $\mathbf{K}$ to $\mathbf{L}$. These transitions are consistent with the order relations on $\mathbf{L}$ and $\mathbf{K}$, as the following result shows.

Lemma 5.21. Let $x<y \in \mathbf{L}$ and $X, Y \in \mathbf{K}$.

(i) If $x \rightarrow X$ and $y \rightarrow Y$, then $X<Y$.

(ii) Similarly, if $X \rightarrow x$ and $Y \rightarrow y$, then $X<Y$.

Proof. (i) Firstly, assume that $x$ unlocks $X$ by convexity, hence $x=x_{1}$ and $X \in V\left(x_{2}\right) \backslash V\left(x_{1}\right)$. If $Y$ were in $V\left(x_{2}\right) \backslash V\left(x_{1}\right)$, then $Y$ would be hidden by $x$, hence not unlocked by $y$. Therefore $Y$ is on the right of $X$, see Figure 15 left. The case $y$ unlocks $Y$ by convexity is the same one.
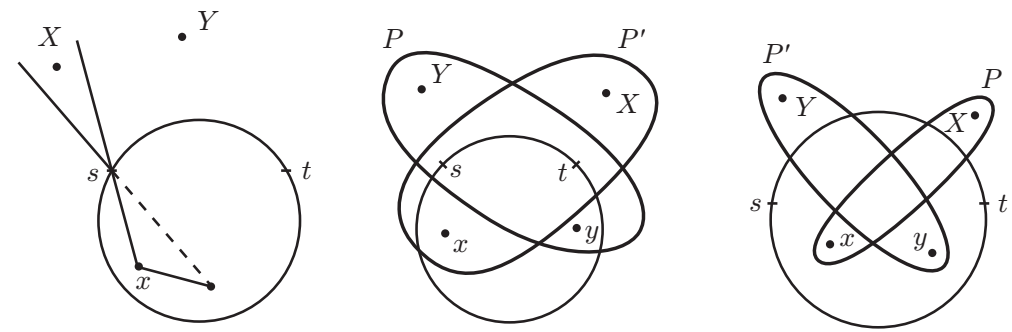

Figure 15: Proof of Lemma 5.21.

Assume now that $x$ and $y$ unlock $X$ and $Y$ by compatibility, and let $P, P^{\prime} \in \mathcal{F}$ be such that $x \underset{P}{\rightarrow} X$ and $y \underset{P^{\prime}}{\rightarrow} Y$. We have $c_{P}(X)=\{x\}$ and $c_{P^{\prime}}(Y)=\{y\}$, hence $s, t \in P \cap P^{\prime}$. We also have $Y \in P$, otherwise $Y$ would be locked by $P$ (with $s \in l_{P}(Y), x \in c_{P}(Y)$, and $t \in r_{P}(Y)$ ), contradicting $y \rightarrow Y$. Similarly, we have $X \in P^{\prime}$. To sum up, we have $[x, X] \subset \operatorname{conv}\left(P^{\prime} \backslash P\right)$, $[y, Y] \subset \operatorname{conv}\left(P \backslash P^{\prime}\right)$ and $x<y$. The compatibility of $P$ and $P^{\prime}$ then implies $X<Y$, see Figure 15 middle.

(ii) In the same way, if $X \underset{P}{\rightarrow} x$ and $Y \underset{P^{\prime}}{\rightarrow}$, then $x, X \in P \backslash P^{\prime}, y, Y \in P^{\prime} \backslash P$, hence 
]$x, X[\cap] y, Y\left[=\emptyset\right.$ by compatibility of $P$ and $P^{\prime}$, therefore $x<y$ implies $X<Y$, see Figure 15 right.

Lemma 5.22. Let $y \in \mathbf{L}, Y_{1}<Y_{2} \in \mathbf{K}$, and $P \in \mathcal{F}$ be such that $y \rightarrow Y_{1} \rightarrow \underset{P}{\rightarrow} Y_{2}$. Then $s \notin P$ (if $Y_{1}>Y_{2}$, then $\left.t \notin P\right)$.

Proof. By contradiction, we assume that $s \in P$. Since $l_{P}\left(Y_{2}\right)=\left\{Y_{1}\right\}$, we have $s \in r_{P}\left(Y_{2}\right)$.

Case 1. $Y_{1}$ is locked by convexity. Since $Y_{1}<Y_{2}$, we have $y=x_{1}, Y_{1} \in\left(x_{1} s\right)^{+}$, and $Y_{2} \in\left(x_{1} s\right)^{-}$. Then we have

$$
\emptyset \neq c_{P}\left(Y_{2}\right) \subseteq C\left(s, Y_{2}, Y_{1}\right) \cap\left(s Y_{1}\right)^{+} \cap \widetilde{P_{0}} \subseteq\left(x_{1} s\right)^{+} \cap \widetilde{P_{0}}=\emptyset
$$

a contradiction.

Case 2. $y$ unlocks $Y_{1}$ by compatibility. Let $Q \in \mathcal{F}$ locking $Y_{1}$. We have $y \in\left(s Y_{1}\right)^{-}$, hence $y \notin c_{P}\left(Y_{2}\right)$, hence $y \in P$.

Choose $u \in c_{P}\left(Y_{2}\right)$, hence $u \neq y$. Since $c_{Q}\left(Y_{1}\right)=\{y\}$, we have $s, u \in Q$. Since $Y_{2} \notin$ $C\left(s, Y_{1}, y\right)$ and $] s, Y_{1}[\cap] u, Y_{2}[\neq \emptyset] y,, Y_{1}[$ crosses $] u, Y_{2}[$. By compatibility of $P$ and $Q$, it follows that $Y_{2} \notin Q$.

Now choose $V \in r_{Q}\left(Y_{1}\right)$. We prove below that $] s, V[$ and $] y, Y_{2}\left[\right.$ cross. Since $s \in l_{Q}\left(Y_{1}\right)$, we have $V \in C\left(Y_{1}, s, y\right)$. Since $Y_{1}<Y_{2}$ and $\mathrm{y} \in \mathbf{L}$, we also have $Y_{2} \in C\left(Y_{1}, s, y\right)$. By Lemma 5.19 (ii), $Y_{1}$ and $Y_{2}$ are consecutive for the order on $\mathbf{K}$. By Lemma 5.16 (ii), it follows that $V \notin \operatorname{conv}\left(s, Y_{1}, Y_{2}\right)$.

If $V$ were in $C\left(Y_{1}, s, Y_{2}\right) \cap\left(u Y_{1}\right)^{+}$, then $Y_{1}$ would be in $\operatorname{conv}(s, u, V) \subseteq \operatorname{conv}(Q)$, a contradiction with $Y_{1} \notin Q$.

If $V$ were in $C\left(Y_{1}, s, Y_{2}\right) \cap\left(u Y_{1}\right)^{-}$, then $] u, V$ [ would cross ] $y, Y_{1}[$, hence, by compatibility of $P$ and $Q, V$ would be in $P$, hence in $l_{P}\left(Y_{2}\right)$, contradicting $Y_{1} \rightarrow Y_{2}$.

To sum up, we have $V \in C\left(Y_{1}, s, y\right) \backslash C\left(Y_{1}, s, Y_{2}\right)$, hence $V \in C\left(Y_{2}, s, y\right)$. Moreover $V \notin$ $\operatorname{conv}\left(s, y, Y_{2}\right)$, otherwise $Y_{2}$ would be hidden, therefore $] s, V[$ and $] y, Y_{2}[$ cross.

Since $y \in \widetilde{P_{0}} \backslash Q$ and $s, V \in Q \backslash P_{0}$, we obtain that $Y_{2}$ is locked by $Q$, in contradiction with $Y_{1} \rightarrow Y_{2}$.

Lemma 5.23. The orbits of the map $\rightarrow$ are of the form

$$
y_{1} \rightarrow y_{2} \rightarrow \cdots \rightarrow y_{n} \rightarrow Y_{1} \rightarrow Y_{2} \rightarrow \cdots \rightarrow Y_{N} \rightarrow y_{1}
$$

with $n, N \geq 1$, the $y_{i}$ consecutive in $\mathbf{L}$, and the $Y_{i}$ consecutive in $\mathbf{K}$.

Proof. Consider an orbit of $\rightarrow$. Recall that, by Lemmas 5.19 and 5.20, this orbit cannot stay entirely in $\mathbf{L}$ or in $\mathbf{K}$. We have to show that it makes exactly one round trip between $\mathbf{K}$ and $\mathbf{L}$. Let $\rightarrow y_{1} \rightarrow y_{2} \rightarrow \cdots \rightarrow y_{n} \rightarrow$ be a maximal portion in $\mathbf{L}$. From Lemma 5.19 (i), it is formed of consecutive points. Suppose $y_{1}<y_{2}<\cdots<y_{n}$ to fix ideas. Let $Y_{1} \in \mathbf{K}$ be unlocked by $y_{n}$ and $Y_{1} \rightarrow \cdots \rightarrow Y_{N} \rightarrow$ be the maximal portion of orbit in $\mathbf{K}$ beginning with $Y_{1}$. They are also consecutive by Lemma 5.19 (ii). Finally, let $y \in \mathbf{L}$ be unlocked by $Y_{N}$. We have to show that $y=y_{1}$. Notice that $y \notin\left\{y_{2}, \ldots, y_{n}\right\}$ by injectivity of $\rightarrow$.

By contradiction, if $y \neq y_{1}$, two cases are possible. If $y<y_{1}$, then the portion of the orbit in $\mathbf{L}$ beginning with $y$ is made of consecutive points, none of them being $y_{1}$, hence entirely on the left of $y_{1}$. When this orbit returns in $\mathbf{K}$, it is at a point on the left of $Y_{1}$ by Lemma 5.21 (i). In the same manner, the portion of orbit in $\mathbf{K}$ is on the left of $Y_{1}, \ldots, Y_{N}$, hence, by Lemma 5.21 (ii), when it returns in $\mathbf{L}$ it is on the left of $y$, hence of $y_{1}$. In this manner, all the sequel of the orbit in $\mathbf{L}$ remains on the left of $y_{1}$, preventing the orbit to close, see Figure 16. The case $y_{1}<y$ is similar, replacing 'left' by 'right'. 


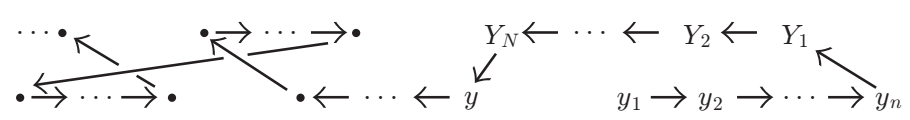

Figure 16: Proof of Lemma 5.23.

\subsection{End of proof of Theorem 3.7}

Notation: $\widetilde{\mathbf{K}}=\mathbf{K} \cup\{s, t\}$ and $\widetilde{\mathbf{L}}=\mathbf{L} \cup\{s, t\}$.

It remains to prove that orbits of $\rightarrow$, as described in Lemma 5.23, are impossible. Up to now it is possible that several orbits coexist. Consider an orbit (with $y_{i} \in \mathbf{L}$ and $Y_{i} \in \mathbf{K}$ )

$$
y_{1} \underset{P_{1}}{\rightarrow} y_{2} \underset{P_{2}}{\rightarrow} \cdots \underset{P_{n-1}}{\rightarrow} y_{n} \underset{Q}{\rightarrow} Y_{1} \underset{P_{1}^{\prime}}{\rightarrow} Y_{2} \underset{P_{2}^{\prime}}{\rightarrow} \cdots \underset{P_{N-1}^{\prime}}{\rightarrow} Y_{N} \underset{Q^{\prime}}{\rightarrow} y_{1} .
$$

All unlockings are by compatibility, except possibly $y_{n} \rightarrow Y_{1}$. In that case, by convention, the notation $y_{n} \underset{Q}{\rightarrow} Y_{1}$ stands for $y_{n} \rightarrow Y_{1}$. By symmetry, we assume without loss that $y_{1}<y_{2}<$ $\cdots<y_{n}$. The orbit is called crossed, if $n, N \geq 2$ and $Y_{1}<Y_{2}<\cdots<Y_{N}$; Otherwise it is called uncrossed. Observe that, if the orbit is uncrossed, then $y_{n}$ can unlock $Y_{1}$ by compatibility or by convexity, but if the orbit is crossed then $y_{n}$ must unlock $Y_{1}$ by compatibility.

Because $x \rightarrow y \rightarrow x$ is impossible, one cannot have at the same time $n=N=1$. We will prove that the assumption $n \geq 2$ implies $N \geq 2$ and, symmetrically, that $N \geq 2$ implies $n \geq 2$.

We first assume that $n \geq 2$. The proof is divided in several steps.

Step 1. We have $s \in P_{1}$.

Proof. Otherwise, we have $s \in r_{P_{1}}\left(y_{2}\right)$. Consider some $Y \in c_{P_{1}}\left(y_{2}\right)$. We have $Y_{N} \in\left(y_{2} s\right)^{-}$hence $Y_{N} \notin c_{P_{1}}\left(y_{2}\right)$, therefore $Y_{N} \notin P_{1}$. As a consequence, we have $Y_{N}, y_{1} \in Q^{\prime} \backslash P_{1}, Y \in P_{1} \backslash Q^{\prime}$, and $y_{2} \in P_{1}$. Since $Y_{N}$ is unlocked by a point which is not $y_{1}$, we have $Y_{N} \in\left(y_{1} s\right)^{-}$, hence ]$Y_{N}, y_{1}[\cap] Y, y_{2}\left[\neq \emptyset\right.$. Together with the compatibility of $Q^{\prime}$ and $P_{1}$, this implies $y_{2} \in Q^{\prime}$. Since $Y_{N} \in\left(y_{1} s\right)^{-}$and $s \notin Q^{\prime}$, we have $s \in l_{Q^{\prime}}\left(y_{1}\right)$. Let $z \in r_{Q^{\prime}}\left(y_{1}\right)$. We have $] Y_{N}, y_{1}[\cap] s, z[\neq \emptyset$. Since $z \notin Q^{\prime}$, we also have $z \notin \operatorname{conv}\left(y_{1}, y_{2}, Y_{N}\right)$. Since $y_{1}$ and $y_{2}$ are consecutive, this implies ]$Y_{N}, y_{2}[\cap] s, z\left[\neq \emptyset\right.$. By Remark 5.2, $Q^{\prime}$ locks $y_{2}$, in contradiction with $y_{1} \rightarrow y_{2}$.

Step 2. We have $P_{1} \cap \widetilde{\mathbf{K}} \subseteq P_{2} \cap \widetilde{\mathbf{K}} \subseteq \cdots \subseteq P_{n-1} \cap \widetilde{\mathbf{K}}$.

Proof. If $n=2$, there is nothing to prove. Otherwise, consider $i \in\{1, \ldots, n-2\}$. We first prove by contradiction that $y_{i+2} \notin P_{i}$. Otherwise we have $y_{i+2} \notin r_{P_{i}}\left(y_{i+1}\right)$. Consider $X \in c_{P_{i}}\left(y_{i+1}\right)$ and $x \in r_{P_{i}}\left(y_{i+1}\right)$; Then we have

$$
\left.\operatorname{conv}\left(X, y_{i+1}, y_{i+2}\right) \subseteq \operatorname{conv}\left(P_{i}\right) \text { and }\right] y_{i+1}, X[\cap] y_{i}, x[\neq \emptyset .
$$

Since $y_{i}, y_{i+1}, y_{i+2}$ are consecutive, the quadrilateral $y_{i} y_{i+1} y_{i+2} x$ is convex in this order, hence we have $] y_{i+2}, X[\cap] y_{i}, x\left[\neq \emptyset\right.$, therefore $P_{i}$ would lock $y_{i+2}$, contradicting $y_{i+1} \rightarrow y_{i+2}$.

We now prove that $y_{i} \in P_{i+1}$, again by contradiction. Otherwise, since $l_{P_{i+1}}\left(y_{i+2}\right)=\left\{y_{i+1}\right\}$, we have $y_{i} \in r_{P_{i+1}}\left(y_{i+2}\right)$. Let $X^{\prime} \in c_{P_{i+1}}\left(y_{i+2}\right)$. We have $] y_{i+2}, X^{\prime}[\cap] y_{i}, y_{i+1}[\neq \emptyset$, hence $y_{i} \notin\left(y_{i+1} X^{\prime}\right)^{+}$. Since $l_{P_{i}}\left(y_{i+1}\right)=\left\{y_{i}\right\}$, by Proposition 5.3 (i) we have $y_{i} \in\left(y_{i+1} Y\right)^{+}$for all $Y \in$ $c_{P_{i}}\left(y_{i+1}\right)$, hence $X^{\prime} \notin c_{P_{i}}\left(y_{i+1}\right)$, hence $X^{\prime} \notin P_{i}$. Now let $X^{\prime \prime} \in c_{P_{i}}\left(y_{i+1}\right)$. In the same manner, we have $X^{\prime \prime} \in\left(y_{i} y_{i+1}\right)^{+}$, hence $X^{\prime \prime} \in\left(y_{i} y_{i+2}\right)^{+}$, hence $X^{\prime \prime} \notin c_{P_{i+1}}\left(y_{i+2}\right)$, hence $X^{\prime \prime} \notin P_{i+1}$. To sum up, we have $X^{\prime}, y_{i+2} \in P_{i+1} \backslash P_{i}, X^{\prime \prime}, y_{i+1} \in P_{i} \backslash P_{i+1}$ and $] X^{\prime \prime}, y_{i+1}[\cap] X^{\prime}, y_{i+2}[\neq \emptyset$, contradicting $P_{i}$ and $P_{i+1}$ compatible. This proves that $y_{i} \in P_{i+1}$.

Altogether, one has $y_{i}, y_{i+2} \in P_{i+1} \backslash P_{i}, y_{i+1} \in P_{i} \backslash P_{i+1}$, and $] Y, y_{i+1}[\cap] y_{i}, y_{i+2}[\neq \emptyset$ for all $Y \in \widetilde{\mathbf{K}}$. Since $P_{i}$ and $P_{i+1}$ are compatible, this implies $P_{i} \cap \widetilde{\mathbf{K}} \subseteq P_{i+1} \cap \widetilde{\mathbf{K}}$.

A consequence of Steps 1 and 2 is that $s \in P_{n-1}$.

Step 3. We have $Y_{N} \in P_{1}$. 
Proof. As in Step 1, we have $Y_{N} \in\left(y_{1} s\right)^{-}$, and we see that $y_{2} \in Q^{\prime}$ would imply $Q^{\prime}$ locks $y_{2}$, contradicting $y_{1} \rightarrow y_{2}$. It follows that $s, y_{2} \in P_{1} \backslash Q^{\prime}, y_{1} \in Q^{\prime} \backslash P_{1}, Y_{N} \in Q^{\prime}$, and $] s, y_{2}[\cap] y_{1}, Y_{N}[\neq$ $\emptyset$. The compatibility of $P_{1}$ and $Q^{\prime}$ now implies $Y_{N} \in P_{1}$.

Step 4. We have $Y_{1} \notin P_{n-1}$.

Proof. Two cases occur: $y_{n}$ unlocks $Y_{1}$ by convexity or by compatibility.

If $y_{n}$ unlocks $Y_{1}$ by convexity, then $y_{n}=x_{m}$, the last point of $\mathbf{L}$. Let $z \in r_{P_{n-1}}\left(y_{n}\right)$. We have $s \in P_{n-1} \cap \widetilde{\mathbf{K}} \subseteq c_{P_{n-1}}\left(y_{n}\right) \subset\left(y_{n} z\right)^{+}$. Because $y_{n}=x_{m}$ and $t$ are two consecutive extreme points of $\operatorname{conv}\left(\widetilde{P_{0}}\right)$, we have $z=t$ or $z \in\left(y_{n} t\right)^{+}$. Since $s \in\left(y_{n} t\right)^{+} \cap\left(y_{n} z\right)^{+}$, we have $\left(y_{n} t\right)^{-} \cap(s t)^{+} \subseteq\left(y_{n} z\right)^{-}$. Therefore the point $Y_{1}$, which is in $\left(y_{n} t\right)^{-} \cap(s t)^{+}$, is also in $\left(y_{n} z\right)^{-}$. This implies $Y_{1} \notin P_{n-1}$, see Figure 17 left.

If $y_{n}$ unlocks $Y_{1}$ by compatibility, with a subset $Q \in \mathcal{F}$, then one has $\left\{y_{n}\right\}=c_{Q}\left(Y_{1}\right)=\widetilde{P_{0}} \backslash Q$. Let $z \in r_{P_{n-1}}\left(y_{n}\right)\left(z=t\right.$ is possible). One has $y_{n-1}, z \in Q \backslash P_{n-1}, y_{n} \in P_{n-1} \backslash Q, Y_{1} \notin Q$, and ]$y_{n-1}, z[\cap] y_{n}, Y_{1}\left[\neq \emptyset\right.$. By compatibility of $P_{n-1}$ and $Q$, we deduce $Y_{1} \notin P_{n-1}$, see Figure 17 right.
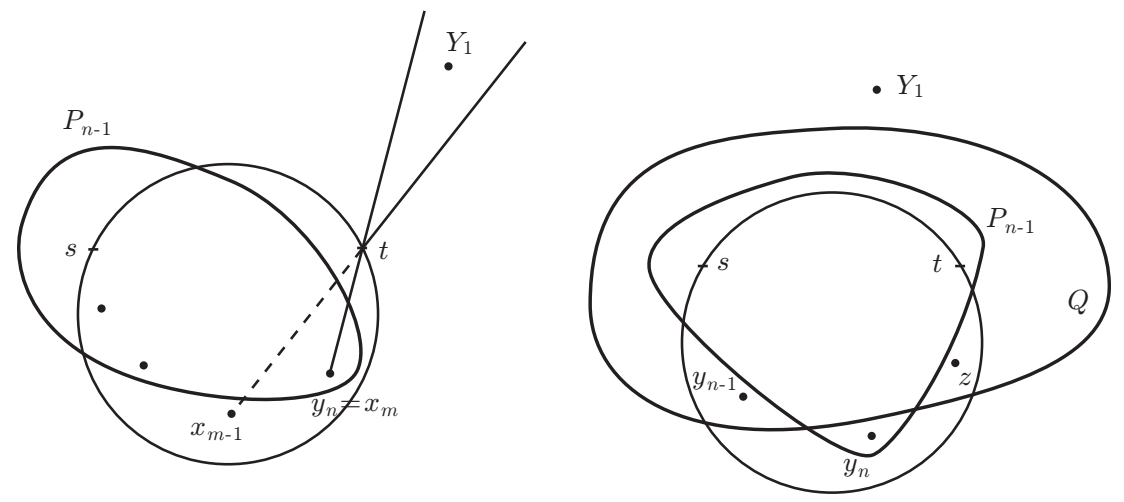

Figure 17: Proof of Step 4.

These first four steps can be summarized in the following formula

$$
s, Y_{N} \in P_{1} \cap \widetilde{\mathbf{K}} \subseteq P_{2} \cap \widetilde{\mathbf{K}} \subseteq \cdots \subseteq P_{n-1} \cap \widetilde{\mathbf{K}} \not \ngtr Y_{1} .
$$

As a consequence, one has $Y_{1} \neq Y_{N}$, hence $N \geq 2$, as announced at the beginning of the subsection. From now on, we no longer assume $n \geq 2$.

Step 5. Symmetrically, we have

$$
y_{n} \notin P_{1}^{\prime} \cap \widetilde{\mathbf{L}} \supseteq P_{2}^{\prime} \cap \widetilde{\mathbf{L}} \supseteq \cdots \supseteq P_{N-1}^{\prime} \cap \widetilde{\mathbf{L}} \ni y_{1} .
$$

Moreover, in the case of a crossed orbit, we have $s \notin P_{1}^{\prime}$ and, in the case of an uncrossed orbit, we have $t \notin P_{1}^{\prime}$. As a consequence, we have $y_{1} \neq y_{n}$, hence $n \geq 2$.

The proof of Step 5 follows the same lines as those of the first four steps. However, since there are some differences, we prefer to give a complete proof. We divide it in four substeps, corresponding to Steps 1 to 4 above.

Substep 5.1. If the orbit is crossed, then $s \notin P_{i}^{\prime}$ for all $i=1, \ldots, N$. If the orbit is uncrossed, then $t \notin P_{i}^{\prime}$ for all $i=1, \ldots, N$. This follows from Lemma 5.22 and Lemma 5.12.

Substep 5.2. We have $P_{1}^{\prime} \cap \widetilde{\mathbf{L}} \supseteq P_{2}^{\prime} \cap \widetilde{\mathbf{L}} \supseteq \cdots \supseteq P_{N-1}^{\prime} \cap \widetilde{\mathbf{L}}$. 
Proof. We consider the case of an uncrossed orbit; The case of a crossed orbit is similar. If $N=2$, then there is nothing to prove. Otherwise, consider $i \in\{1, \ldots, N-2\}$.

We first prove that $Y_{i} \notin P_{i+1}^{\prime}$. By the definition of the order in $\mathbf{K}$, we have $Y_{i} \in\left(t Y_{i+2}\right)^{-}$ and, by Substep 5.1, $t \in c_{P_{i+1}^{\prime}}\left(Y_{i+2}\right)$, hence $Y_{i}$ cannot be in $l_{P_{i+1}^{\prime}}\left(Y_{i+2}\right)$. Since $r_{P_{i+1}^{\prime}}\left(Y_{i+2}\right)$ is reduced to $Y_{i+1}$, we deduce that $Y_{i} \notin P_{i+1}^{\prime}$.

We now prove by contradiction that $Y_{i+2} \in P_{i}^{\prime}$. Choose $X \in l_{P_{i}^{\prime}}\left(Y_{i+1}\right)$ and $Z \in l_{P_{i+1}^{\prime}}\left(Y_{i+2}\right)$. By Lemma 5.16 (ii), $X$ cannot be in conv $\left(t, Y_{i+1}, Y_{i+2}\right)$. It follows that, either $] Y_{i+1}, Z[\cap] X, Y_{i}[\neq$ $\emptyset$, or $] t, Y_{i+2}[\cap] X, Y_{i}\left[\neq \emptyset\right.$. In the latter case, $P_{i}$ would lock $Y_{i+2}$, a contradiction with $Y_{i+1} \rightarrow$ $Y_{i+2}$, hence we have $] Y_{i+1}, Z[\cap] X, Y_{i}[\neq \emptyset$.

Suppose that $X \in P_{i+1}^{\prime}$. Then, since $r_{P_{i+1}^{\prime}}\left(Y_{i+2}\right)=\left\{Y_{i+1}\right\}$, we would have $X \in l_{P_{i+1}^{\prime}}\left(Y_{i+2}\right) \subset$ $\left(t Y_{i+2}\right)^{+}$. Since $Y_{i+2} \in\left(X Y_{i+1}\right)^{-}$, this implies $Y_{i+2} \in \operatorname{conv}\left(X, Z, Y_{i+1}\right) \subseteq \operatorname{conv}\left(P_{i+1}\right)$, a contradiction with $Y_{i+2} \notin P_{i+1}$. Therefore we have $X \notin P_{i+1}^{\prime}$.

Now the compatibility of $P_{i}^{\prime}$ and $P_{i+1}^{\prime}$ implies that $Z \in P_{i}^{\prime}$ and therefore $Z \in l_{P_{i}^{\prime}}\left(Y_{i+1}\right)$. It follows that $P_{i}^{\prime}$ locks $Y_{i+2}$, a contradiction. This proves that $Y_{i+2} \in P_{i}^{\prime}$.

Finally, we prove that $P_{i+1}^{\prime} \cap \widetilde{\mathbf{L}} \subseteq P_{i}^{\prime} \cap \widetilde{\mathbf{L}}$. Otherwise, choose $u \in\left(P_{i+1}^{\prime} \backslash P_{i}^{\prime}\right) \cap \widetilde{\mathbf{L}}$. By Proposition 5.17 we have $] u, Y_{i+1}[\cap] Y_{i}, Y_{i+2}\left[\neq \emptyset\right.$, contradicting the compatibility of $P_{i}^{\prime}$ and $P_{i+1}^{\prime}$.

Substep 5.3. We have $y_{1} \in P_{N-1}^{\prime}$.

Proof. We consider the case of an uncrossed orbit; The case of a crossed orbit is similar. By Substep 5.1, $t$ is in none of the $P_{i}^{\prime}$. Since $Y_{N} \underset{Q^{\prime}}{\rightarrow} y_{1}$, by Lemma 5.11 we have $s \in P_{N-1}^{\prime}$. To sum up, we have $y_{1} \in Q^{\prime}, Y_{N} \in Q^{\prime} \backslash P_{N-1}^{\prime}, s, Y_{N-1} \in P_{N-1}^{\prime} \backslash Q^{\prime}$, and $] y_{1}, Y_{N}[\cap] s, Y_{N-1}[\neq \emptyset$, hence, by compatibility of $P_{N-1}^{\prime}$ and $Q^{\prime}, y_{1} \notin P_{N-1}^{\prime}$.

Substep 5.4. We have $y_{n} \notin P_{1}^{\prime}$.

Proof. We consider the case of an uncrossed orbit; The case of a crossed orbit is similar. By Step 4, we have $Y_{1} \notin P_{n-1}$.

If $y_{n}$ unlocks $Y_{1}$ by convexity, then choose $X \in l_{P_{1}^{\prime}}\left(Y_{2}\right)$. By Substep 5.1, we have $t \notin P_{1}^{\prime}$, hence $t \in c_{P_{1}^{\prime}}\left(Y_{2}\right)$, hence $X \in C\left(t, Y_{1}, Y_{2}\right) \cap\left(t Y_{2}\right)^{+}$. We also have $y_{n} \in\left(t Y_{1}\right)^{-} \cap\left(t Y_{2}\right)^{+}$, hence $t \in \operatorname{conv}\left(X, Y_{1}, y_{n}\right)$. Since $t \notin P_{1}^{\prime}$, this implies $y_{n} \notin P_{1}^{\prime}$.

If $y_{n}$ unlocks $Y_{1}$ by compatibility, with a subset $Q \in \mathcal{F}$, then $\left\{y_{n}\right\}=c_{Q}\left(Y_{1}\right)=\widetilde{P_{0}} \backslash Q$, $s, t \in Q, s \in l_{Q}\left(Y_{1}\right)$, and $t \in r_{Q}\left(Y_{1}\right)$. If $Y_{2}$ were not in $Q$, then $Q$ would lock $Y_{2}$, contradicting $Y_{1} \rightarrow Y_{2}$, hence $Y_{2} \in Q \backslash P_{1}^{\prime}$. By Substep 5.1, we also have $t \notin P_{1}^{\prime}$. To summarize, we have $Y_{2} \in Q \backslash P_{1}^{\prime}, Y_{1} \in P_{1}^{\prime} \backslash Q, y_{n} \notin Q$, and $] y_{n}, Y_{1}[\cap] t, Y_{2}\left[\neq \emptyset\right.$, hence $y_{n} \notin P_{1}^{\prime}$ by compatibility of $Q$ and $P_{1}^{\prime}$.

Step 6. Once again, we distinguish the cases of a crossed or an uncrossed orbit.

Substep 6.1. In the case of a crossed orbit, we prove below by induction that, for all $i \in$ $\{1, \ldots, n\}$, one has $y_{i} \in P_{1}^{\prime}$, in contradiction with (17).

By (17), the property is satisfied for $i=1$. If the property is satisfied for $i<n$, then we have $y_{i}<y_{i+1}$. Since the orbit is crossed, $Y_{1}$ is not locked by convexity, hence the quadrilateral $s y_{i} y_{i+1} Y_{1}$ is convex, hence $] s, y_{i+1}[\cap] Y_{1}, y_{i}\left[\neq \emptyset\right.$; We also have $Y_{1}, y_{i} \in P_{1}^{\prime} \backslash P_{i}, s \in P_{i} \backslash P_{1}^{\prime}$ and $y_{i+1} \in P_{i}$. The compatibility of $P_{1}^{\prime}$ and $P_{i}$ then implies $y_{i+1} \in P_{1}^{\prime}$.

Substep 6.2. In the case of an uncrossed orbit, we look for $i<n$ (i.e. $i \in\{1, \ldots, n-1\}$ ) and $I<N$ such that the subsets $P_{i}$ and $P_{I}^{\prime}$ are incompatible. We already have, for all $i<n$ and all $I<N, y_{i}<y_{i+1}$ and $Y_{I+1}<Y_{I}$, hence the pentagon $s y_{i} y_{i+1} Y_{I} Y_{I+1}$ is convex, hence 
]$y_{i}, Y_{I}[\cap] y_{i+1}, Y_{I+1}\left[\neq \emptyset\right.$. One also has $y_{i} \notin P_{i}, y_{i+1} \in P_{i}, Y_{I} \in P_{I}^{\prime}$ and $Y_{I+1} \notin P_{I}^{\prime}$. Therefore we look for $i<n$ and $I<N$ such that

$$
y_{i} \in P_{I}^{\prime}, \quad y_{i+1} \notin P_{I}^{\prime}, \quad Y_{I} \notin P_{i}, \quad Y_{I+1} \in P_{i} .
$$

For $I<N$, set $f(I)=\max \left\{i<n ; \forall k \leq i, y_{k} \in P_{I}^{\prime}\right\}$. Since $y_{1} \in P_{I}^{\prime}$ and $y_{n} \notin P_{I}^{\prime}$, one has $y_{f(I)} \in P_{I}^{\prime}$ et $y_{f(I)+1} \notin P_{I}^{\prime}$. Similarly, for $i<n$, let $g(i)=\max \left\{I<N ; \forall k \leq I, Y_{k} \notin P_{i}\right\}$. Because $Y_{1} \notin P_{i}$ and $Y_{N} \in P_{i}$, we have $Y_{g(i)} \notin P_{i}$ and $Y_{g(i)+1} \in P_{i}$. Using (16), resp. (17), it is straightforward that $f$ is nonincreasing from $\{1, \ldots, N-1\}$ to $\{1, \ldots, n-1\}$, resp. $g$ is nonincreasing from $\{1, \ldots, n-1\}$ to $\{1, \ldots, N-1\}$. Then the product $f \circ g$ is a nondecreasing map from the finite set $\{1, \ldots, n-1\}$ into itself, and thus has at least one fixed point $i$. The pair $(i, I)$ with $I=g(i)$ satisfies (18), yielding $P_{i}$ and $P_{I}^{\prime}$ incompatible; This completes the proof of Theorem 3.7.

\section{Miscellaneous related results and remarks}

\subsection{Cake number and VC-dimension}

Given integers $m, n \geq 1$, the cake number $c(m, n)$ is the maximal number of pieces obtained by cutting an $m$-dimensional cake in $n$ cuts, i.e. the maximal number of connected components of $\mathbb{R}^{m} \backslash \cup_{i=1}^{n} H_{i}$, where $H_{i}$ are affine hyperplanes of $\mathbb{R}^{m}$. It is known that $c(m, n)=\sum_{k=0}^{m}\left(\begin{array}{l}n \\ k\end{array}\right)$ (with the convention $\left(\begin{array}{l}n \\ k\end{array}\right)=0$ if $n<k$, hence $c(m, n)=2^{n}$ if $n \leq m$ ), see e.g. [17, 20] in the case $m=3$, and $[11,21,22]$ in the general case. It is noticeable that the bound is reached for any configuration of hyperplanes in general position, i.e. when the intersection of any $m+1$ of them is empty.

The cake number also appears in the theory of VC-dimension introduced by Vapnik and Chervonenkis in [19]. Given a finite set $S$ and a family $\mathcal{F}$ of subsets of $S$, a subset $A$ of $S$ is said to be shattered by $\mathcal{F}$ if, for every subset $B$ of $A$, there exists $T \in \mathcal{F}$ with $B=T \cap A$. The maximal size of a subset of $S$ shattered by $\mathcal{F}$ is called the $V C$-dimension of $\mathcal{F}$. The fundamental result of this theory (see also $[14,16]$ ) is the following: If $\mathcal{F}$ has VC-dimension at most $d$, then $|\mathcal{F}| \leq c(d,|S|)$. An example of family reaching the bound is the family of all subsets of $S$ of size at most $d$.

We recall the following result of Buzaglo, Holzman, and Pinchasi, Theorem 7 of [4], already mentioned in our introduction. Let $S \subset \mathbb{R}^{2}$ be a finite set and let $\mathcal{F}$ be a family of subsets of $S$ separable by Jordan curves $\left(\gamma_{i}\right)_{1 \leq i \leq|\mathcal{F}|}$. Let $s \geq 2$ be an even integer. If any two curves $\gamma_{i}, \gamma_{j}$ intersect properly at most $s$ times and if $\operatorname{disk}\left(\gamma_{i}\right) \cap \operatorname{disk}\left(\gamma_{j}\right)$ is either empty or connected, then $\mathcal{F}$ has VC-dimension at most $s+1$.

Observe that, in the case of pseudo-circles, convex or not, the condition $\operatorname{disk}\left(\gamma_{i}\right) \cap \operatorname{disk}\left(\gamma_{j}\right)$ empty or connected is automatically satisfied. A nontrivial consequence of this result and of Example 1 of the forthcoming Section 6.2 is that a maximal family of subsets of $S$ separable by circles is also maximal for pseudo-circles, when $S$ is in general position.

Let us mention a short proof that a family $\mathcal{F}$ separable by convex pseudo-circles has VCdimension at most 3, i.e. that a subset $A=\{p, q, r, s\}$ of four points of $S$ cannot be shattered by $\mathcal{F}$. Two cases occur. If the points are not in convex position, then one of the points, say $p$, is in the convex hull of $q, r, s$ and the set $\{q, r, s\}$ cannot be written as $A \cap T$ with $T \in \mathcal{F}$. Otherwise the two diagonals, say $[p, r]$ and $[q, s]$, intersect. By Proposition 2.4, the subsets $\{p, r\}$ and $\{q, s\}$ cannot be written as $A \cap T_{1}$, resp. $A \cap T_{2}$ with $T_{1}, T_{2}$ convex and compatible. We will generalize this proof in arbitrary dimension in Section 6.3.

\subsection{Separation by functions}

In this section we link the cake number with the number of subsets of a finite set $S$ that are separable by circles or by more general kinds of lines. 
It is well-known and relatively easy to prove that the total number of subsets separable by circles is $\mathcal{O}\left(n^{3}\right)$. The exact value, however seems to be known only by specialists. We found only one reference from which the number $c(3, n)$, even if not explicitely written, can be quickly deduced: the proof of Lemma 13 in [9].

Here $S$ denotes a finite subset of $n$ elements of an arbitrary set $X$. In our applications $X$ will be the euclidean plane or space.

Instead of dealing with affine hyperplanes of $\mathbb{R}^{m}$, we will consider linear hyperplanes of $\mathbb{R}^{d}$ with $d=m+1$, i.e. hyperplanes containing the origin $\overrightarrow{0}$. A collection of linear hyperplanes of $\mathbb{R}^{d}$ are said to be in general position if the intersection of any $d$ of them is $\{\overrightarrow{0}\}$.

Let $E$ be a $d$-dimensional subspace of $\mathbb{R}^{X}$, the vector space of functions from $X$ to $\mathbb{R}$. We fix a non-zero linear form $l: E \rightarrow \mathbb{R}$ and we consider the open half-space $E^{+}$of $E$ defined by

$$
E^{+}=\{f \in E ; l(f)>0\} .
$$

A subset $A$ of $S$ is said to be separable by $E^{+}$if there exists $f \in E^{+}$which is negative on $A$ and positive on $S \backslash A$. Notice that a separating function cannot vanish on $S$.

The following result gives the number of subsets of $S$ that are separable by $E^{+}$. For each $x \in X, l_{x}$ denotes the linear form $l_{x}: E \rightarrow \mathbb{R}, f \mapsto f(x)$. The set $S$ is said to be in general position relatively to $E^{+}$if the linear forms $l_{x}, x \in S$, are all non-zero and if the $n+1$ hyperplanes $H=\{l=0\}$ and $H_{x}=\left\{l_{x}=0\right\}$ are in general position.

Theorem 6.1. If $S$ is in general position relatively to $E^{+}$, then the number of subsets of $S$ that can be separated by $E^{+}$is exactly $c(d-1, n)=\sum_{k=0}^{d-1}\left(\begin{array}{l}n \\ k\end{array}\right)$. In the degenerate cases, this number is at most $c(d-1, n)$.

Proof. We first prove that, in both cases, the number of subsets of $S$ separable by $E^{+}$is the number of connected components of $P=E^{+} \backslash \cup_{x \in S} H_{x}$. Precisely we prove that two functions separate the same subset of $S$ if and only if they belong to the same connected component of $P$.

Let $f, g \in E^{+}$be in the same connected component of $P$, denoted by $Q$. Let $\gamma:[0,1] \rightarrow Q$ be a path in $Q$ joining $f$ to $g$. For each $x \in S$ and any $t \in[0,1]$, we have $\gamma(t) \notin H_{x}$, i.e. $l_{x}(\gamma(t)) \neq 0$. By continuity of $l_{x}$, the sign of $l_{x}(\gamma(t))$ is constant, hence the signs of $l_{x}(f)=f(x)$ and of $g(x)$ are the sames, therefore $f$ and $g$ separate the same subsets of $S$.

Conversely, if $f$ and $g$ separate the same subsets of $S$ then, for all $x \in S, f(x)$ and $g(x)$ have the same sign, hence the function

$$
t \in[0,1] \mapsto l_{x}((1-t) f+t g)=(1-t) f(x)+t g(x)
$$

does not vanish. This yields a path $\gamma$ from $f$ to $g$ within $P$, namely $\gamma(t)=(1-t) f+t g$, showing that $f$ and $g$ are in the same connected component of $P$.

Now consider the intersection of $P$ with the affine hyperplane $H_{1}=\{l=1\}$. The connected components of $P$ are in bijection with the connected components of $H_{1} \backslash \cup_{x \in S} H_{x}$. Conversely any configuration of affine hyperplanes in $\mathbb{R}^{d-1}$ can be seen as the intersection of a configuration of linear hyperplanes in $\mathbb{R}^{d}$, and the properties of general position correspond. This establishes the link with the usual $(d-1)$-dimensional cake number, see e.g. [22].

Examples. 1. Let $X=\mathbb{R}^{2}$ and $E$ be the set of polynomial functions of the form $f(x, y)=$ $a\left(x^{2}+y^{2}\right)+b x+c y+d$. If we choose $l(f)=a$ then, for any $f \in E^{+}$, the subset of $S$ separated by $f$ is $S \cap U$, where $U$ is the disk bounded by the circle of equation $f(x, y)=0$. Since $E$ has dimension 4 , this proves that the number of subsets of $S$ separable by circles is at most $c(3, n)$ in the general case, and that this bound is attained if the points of $S$ are in general position relatively to $E^{+}$, i.e. no three of them collinear and no four of them cocyclic. In Section 6.3, this example will be generalized in arbitrary dimension. 
2. A natural generalization is to choose any strictly convex function $h: \mathbb{R}^{2} \rightarrow \mathbb{R}$ instead of the function $x^{2}+y^{2}$ and to consider the vector space of all functions of the form $f(x, y)=$ $a h(x, y)+b x+c y+d, a, b, c, d \in \mathbb{R}$. This shows that the number of subsets of $S$ separable by the family of convex pseudo-circles obtained as projections on the plane $x y$ of intersections of affine planes with the convex surface $z=h(x, y)$ is still equal to $c(3, n)$, provided the points of $S$ are in general position (here no three collinear and no four on a same curve $a h(x, y)+b x+c y+d=0$ ).

Observe that this kind of family of convex pseudo-circles is very particular. As Rote explains in Har-Peled's blog [13], if $A, B, C$ are three such pseudo-circles, then the three lines passing through $A \cap B$, respectively $B \cap C$ and $C \cap A$, must intersect (since they are projections of intersections of two planes among three in $\mathbb{R}^{3}$ ) whereas these lines have no reason to intersect for general convex pseudo-circles. This explains why a lifting in $\mathbb{R}^{3}$ cannot be used to prove Theorem 3.9, as already said at the end of Subsection 5.1.

3. Once again with $X=\mathbb{R}^{2}$, let $E$ be the set of all polynomial functions of degree at most two, i.e. $E=\left\{f:(x, y) \mapsto a_{1} x^{2}+a_{2} x y+a_{3} y^{2}+a_{4} x+a_{5} y+a_{6} ; a_{i} \in \mathbb{R}\right\}$. The linear form $l$ is arbitrary, e.g. $l$ can be the evaluation at one point $p_{0} \in \mathbb{R}^{2} \backslash S$. Then the number of subsets separable by "conical regions" containing $p_{0}$ is at most $c(5, n)$, and this bound is achieved if no five points are collinear and no six of them are on the same conic. By "conical region", we mean a region, connected or not, defined by a polynomial inequality, with a polynomial of degree at most two.

Since conics intersect at most four times, this result may be compared with the aforementioned result of Buzaglo, Holzman, and Pinchasi (recall that $|\mathcal{F}|=c(5, n)$ in the case $s=4$ ), although conics have no more the connected intersection property and are no more Jordan curves (but they become Jordan curves in the projective plane).

\subsection{Separation by convex pseudo-spheres}

In this section, we first check that the separation by usual spheres satisfies Definition 1.3; Then we prove Theorem 1.4. For the convenience of the reader, the statements are reproduced below.

Definition 1.3 Given a finite subset $S$ of $\mathbb{R}^{d}$, a family $\mathcal{F}$ of subsets of $S$ is said to be separable by convex pseudo-spheres if $\operatorname{conv}(T) \cap S=T$ for all $T \in \mathcal{F}$ and $\operatorname{conv}\left(T \backslash T^{\prime}\right) \cap \operatorname{conv}\left(T^{\prime} \backslash T\right)=\emptyset$ for all $T, T^{\prime} \in \mathcal{F}$.

If a subset $T$ of $S$ is separated from the rest of $S$ by a $(d-1)$-dimensional sphere $\Sigma$, then $\operatorname{conv}(T)$ is in the open ball $U$ of boundary $\Sigma$, hence $T \subseteq \operatorname{conv}(T) \cap S \subseteq U \cap S=T$, and if $T, T^{\prime} \subseteq S$ are separated by two spheres $\Sigma=\partial U$, resp. $\Sigma^{\prime}=\partial U^{\prime}$, then $\operatorname{conv}\left(T \backslash T^{\prime}\right)$ and $\operatorname{conv}\left(T^{\prime} \backslash T\right)$ are separated by the hyperplane containing $\Sigma \cap \Sigma^{\prime}$ (or by an hyperplane separating $U$ and $U^{\prime}$ if $U \cap U^{\prime}=\emptyset$ ), hence do not intersect.

Theorem 1.4 Let $d \geq 2$ and let $S$ be a set of $n$ points in $\mathbb{R}^{d}$ in general position, i.e. no $d+1$ of them in the same hyperplane. Then every family $\mathcal{F}$ of subsets of $S$ separable by convex pseudo-spheres has at most $c(d+1, n)=\left(\begin{array}{l}n \\ 0\end{array}\right)+\left(\begin{array}{l}n \\ 1\end{array}\right)+\cdots+\left(\begin{array}{c}n \\ d+1\end{array}\right)$ elements. If moreover no $d+2$ points of $S$ lie on a same sphere, then the bound is attained for the family of all subsets of $S$ separable by $(d-1)$-dimensional spheres.

Proof of Theorem 1.4 We show that the VC-dimension of $\mathcal{F}$ is at most $d+1$, i.e. that a subset $A=\left\{a_{1}, a_{2}, \ldots, a_{d+2}\right\}$ of $d+2$ points of $S$ cannot be shattered by $\mathcal{F}$. Two cases occur.

If the points of $A$ are not in convex position, then at least one of them, say $a_{1}$, is in the convex hull of the $d+1$ other points of $A$. By convexity, every subset $T$ of $\mathcal{F}$ that contains $a_{2}, \ldots, a_{d+2}$ also contains $a_{1}$, hence the set $\left\{a_{2}, \ldots, a_{d+2}\right\}$ cannot be written as $A \cap T$ with $T \in \mathcal{F}$. 
If the points of $A$ are in convex position, then consider one facet of the polyhedron $\operatorname{conv}(A)$. Assume without loss of generality that this hyperface is $\operatorname{conv}\left(a_{3}, \ldots, a_{d+2}\right)$. The points $a_{1}$ and $a_{2}$ are in the same open half-space delimited by the hyperplane containing $a_{3}, \ldots, a_{d+2}$. Consider a point $M$ in the same half-space, close to an interior point of the hyperface. In this manner, we have $M \in \operatorname{conv}\left(a_{1}, a_{3}, \ldots, a_{d+2}\right) \cap \operatorname{conv}\left(a_{2}, a_{3}, \ldots, a_{d+2}\right)$. Therefore there exist non-negative real constants $\lambda_{1}, \lambda_{3}, \ldots, \lambda_{d+2}$ with sum 1 and $\mu_{2}, \mu_{3}, \ldots, \mu_{d+2}$ also with sum 1 , such that

$$
M=\lambda_{1} a_{1}+\lambda_{3} a_{3}+\cdots+\lambda_{d+2} a_{d+2}=\mu_{2} a_{2}+\mu_{3} a_{3}+\cdots+\mu_{d+2} a_{d+2} .
$$

Moreover, since $M$ is in the open half-space, the numbers $\lambda_{1}$ and $\mu_{2}$ are non-zero. We now use the same trick as in the proof of Lemma 2.10. With the convention $\mu_{1}=\lambda_{2}=0$, set $I=\left\{i \in\{1, \ldots, d+2\} ; \lambda_{i}<\mu_{i}\right\}$ and $J=\left\{i \in\{1, \ldots, d+2\} ; \lambda_{i}>\mu_{i}\right\}$. These are disjoint non-empty subsets of $\{1, \ldots, d+2\}$. We then have $\sum_{i \in I}\left(\mu_{i}-\lambda_{i}\right) a_{i}=\sum_{i \in J}\left(\lambda_{i}-\mu_{i}\right) a_{i}$; We also have $\sum_{i \in I}\left(\mu_{i}-\lambda_{i}\right)=\sum_{i \in J}\left(\lambda_{i}-\mu_{i}\right) \neq 0$. Denoting this quantity by $\kappa$, we obtain a point of $\operatorname{conv}\left\{a_{i} ; i \in I\right\} \cap \operatorname{conv}\left\{a_{i} ; i \in J\right\}$, namely $\frac{1}{\kappa} \sum_{i \in I}\left(\mu_{i}-\lambda_{i}\right) a_{i}$. This shows that the subsets $A_{1}=\left\{a_{i} ; i \in I\right\}$ and $A_{2}=\left\{a_{i} ; i \in J\right\}$ cannot be written as $A \cap T_{1}$, resp. $A \cap T_{2}$ with $T_{1}, T_{2}$ convex and compatible. We proved that, in both cases, $A$ is not shattered by $\mathcal{F}$.

To prove that the bound is achieved by the family of subsets separable by $(d-1)$-dimensional spheres, we apply Theorem 6.1: We choose $X=\mathbb{R}^{d}$ and $l: E \rightarrow \mathbb{R}, f \mapsto a_{0}$, where

$$
E=\left\{f:\left(x_{1}, \ldots x_{d}\right) \mapsto a_{0}\left(x_{1}^{2}+\cdots+x_{d}^{2}\right)+a_{1} x_{1}+\cdots+a_{d} x_{d}+a_{d+1} ; a_{i} \in \mathbb{R}\right\} .
$$

This completes the proof of Theorem 1.4.

\subsection{Further remarks and questions}

6.4.1. We now return to Theorem 1.2. Let $S$ be an $n$-point set. For each $k \in\{1, \ldots, n-1\}$ in the plane, let $b(k, S)$ denote the maximum of the sizes of all families of $k$-subsets of $S$ separable by convex pseudo-circles. Theorem 1.2 asserts that this maximum is achieved by any such family which is maximal for inclusion, and is

$$
b(k, S)=2 k n-n-k^{2}+1-\sum_{i=1}^{k-1} a_{i}(S) .
$$

As we know, we have $\sum_{k=0}^{n} b(k, S)=c(3, n)$, the total size of any maximal family of subsets of $S$ separable by convex pseudo-circles.

A consequence is that, given an integer $k \in\{1, \ldots, n-1\}$ and any maximal family $\mathcal{F}$ of subsets of $S$ separable by convex pseudo-circles, the family of $k$-subsets of $\mathcal{F}$ is already maximal among all families of $k$-subsets of $S$ separable by convex pseudo-circles.

We explain below why this is false without convexity. Let $B(k, n)=\max _{|S|=n} b(k, S)$ and $B(n)=\sum_{k=0}^{n} B(k, n)$. The table below shows the first values of $B(k, n), B(n)$, and $c(3, n)$. As an example, if $S_{1}$ consists of four points in convex position, then $b\left(2, S_{1}\right)=5$ and $b\left(3, S_{1}\right)=$ 4 , whereas if $S_{2}$ consists of four points, one of them in the convex hull of the others, then $b\left(2, S_{2}\right)=6$ and $b\left(3, S_{2}\right)=3$. This explains why $B(4)$ exceeds $c(3,4)$. It is easy to verify that $B(n)>c(3, n)$ for all $n \geq 4$. Since $b(k, S) \leq 2 k n$ we have $B(n)=\mathcal{O}\left(n^{3}\right)$.

Consider now $A(k, S)$, the maximum of the sizes of all families of $k$-subsets of $S$ separable by pseudo-circles, convex or non-convex. Since a diffeomorphism brings $S$ to any other $n$-point set of the plane, and since the property of pseudo-circle is preserved by a diffeomorphism, $A(k, S)$ depends only on $k$ and $n=|S|$. As a consequence, we obtain $A(k, S) \geq B(k, n)$, hence

$$
\sum_{k=0}^{n} A(k, S) \geq B(n)>c(3, n) \text { if } n \geq 4 \text {. }
$$




\begin{tabular}{|c|c|c|c|c|c|c|c|}
\hline$n$ & 0 & 1 & 2 & 3 & 4 & 5 & 6 \\
\hline 0 & 1 & 1 & 1 & 1 & 1 & 1 & 1 \\
1 & & 1 & 2 & 3 & 4 & 5 & 6 \\
2 & & & 1 & 3 & 6 & 9 & 12 \\
3 & & & & 1 & 4 & 7 & $\geq 13$ \\
4 & & & & & 1 & 5 & $\geq 10$ \\
5 & & & & & & 1 & 6 \\
6 & & & & & & & 1 \\
\hline$B(n)$ & 1 & 2 & 4 & 8 & 16 & 28 & $\geq 49$ \\
$c(3, n)$ & 1 & 2 & 4 & 8 & 15 & 26 & 42 \\
\hline
\end{tabular}

Now let $\mathcal{F}$ be any family of subsets of $S$ separable by pseudo-circles (convex or not), which is maximal for inclusion. Since $c(3, n)$ is an upper bound of the size of $\mathcal{F}$, this means that, at least for one $k$, the family of $k$-subsets of $\mathcal{F}$ cannot be maximal among all families of $k$-subsets of $S$ separable by convex pseudo-circles.

Our questions in this direction are the following ones. Do we have $A(k, n)=B(k, n)$ for all $k$ and $n$ ? Do families of $k$-subsets of $S$ separable by pseudo-circles that are maximal for inclusion have the same size $A(k, n)$ ? What is the exact value of $B(n)$ ?

6.4.2. Concerning subsets separable by curves having the $s$-intersection and the connected intersection property in the sense of [4], the situation mainly depends whether we seek for convex curves or not. For example, if $S$ has $n=4$ points, all subsets can be separated by 4 -intersecting curves, whereas for convex curves it depends whether they are in convex or non-convex position. Our questions are as follows: Is $c(5, n)$ achievable by connected and 4-intersecting curves? Is it always achieved by families maximal for inclusion? Is $c(5, n)$ achieved by convex connected and 4 -intersecting curves if $S$ in convex position? In the general case ( $S$ not in convex position) is the number of subsets of $S$ separable by convex connected and 4-intersecting curves equal to number separable by ellipses? The same questions also hold for connected and $s$-intersecting families for any even $s$.

6.4.3. A purely set-theory question: Given an $n$-element set $S$ and a family $\mathcal{F}$ which is maximal for inclusion among families of subsets of $S$ of VC-dimension at most $d$, is it clear that $|\mathcal{F}|=c(d, n)$ ?

Given an $n$-point set $S$ in the plane, among families of subsets of $S$ of VC-dimension at most 3 , which ones are realizable as families separables by pseudo-circles? by convex pseudo-circles? by circles?

Not all families are realizable. In particular maximal families must at least contain all singletons, and the empty set $\emptyset$ and $S$ in its whole since it is always possible to add small circles around every points of $S$ and a big circle around all $S$ and a small one around nothing, which intersect no other pseudo-circle.

Even families containing all singletons of $S, \emptyset$ and $S$ itself are not all realizable. For instance, with $S=\{1,2,3,4,5\}$, let the subsets of $S$ be denoted as numbers, e.g. $S$ itself is denoted by $S=12345$. The family $\mathcal{F}$ containing $\emptyset$ and $S$, all singletons and all doubletons, namely

$$
\mathcal{F}=\{1,2,3,4,5,12,13,14,15,23,24,25,34,35,45,12345\}
$$

has VC-dimension 3 but is not realizable by pseudo-circles, convex or not, as shown in a picture: This comes from the fact that the complete graph $K_{5}$ is not planar.

The same questions arise for VC-dimension $s+1$ and $s$-intersecting families for any even $s$. 


\section{References}

[1] A. Andrzejak and K. Fukuda. Optimization over $k$-set polytopes and efficient $k$-set enumeration. In Proc. 6th Workshop Algorithms Data Struct., volume 1663 of Lecture Notes Comput. Sci., pages 1-12. Springer-Verlag, 1999.

[2] A. Andrzejak and E. Welzl. In between $k$-sets, $j$-facets, and $i$-faces: $(i, j)$-partitions. Discrete Comput. Geom., 29:105-131, 2003.

[3] F. Aurenhammer and O. Schwarzkopf. A simple on-line randomized incremental algorithm for computing higher order Voronoi diagrams. Internat. J. Comput. Geom. Appl., 2:363381, 1992.

[4] S. Buzaglo, R. Holzman, and R. Pinchasi. On $s$-intersecting curves and related problems. In Proc. ACM Sympos. Comput. Geom., pages 79-84, 2008.

[5] Nicolas Chevallier, Augustin Fruchard, Dominique Schmitt, and Jean-Claude Spehner. Separation by convex pseudo-circles. In Proc. 30th Annu. Sympos. Comput. Geom., SOCG'14, pages 444-453, New York, NY, USA, 2014. ACM.

[6] T. K. Dey. Improved bounds on planar $k$-sets and related problems. Discrete Comput. Geom., 19:373-382, 1998.

[7] H. Edelsbrunner, P. Valtr, and E. Welzl. Cutting dense point sets in half. Discrete Comput. Geom., 17:243-255, 1997.

[8] W. El Oraiby, D. Schmitt, and J.-C. Spehner. Centroid triangulations from k-sets. Internat. J. Comput. Geom. Appl., 21(06):635-659, 2011.

[9] D. T. Lee. On $k$-nearest neighbor Voronoi diagrams in the plane. IEEE Trans. Comput., C-31:478-487, 1982.

[10] Y. Liu and J. Snoeyink. Quadratic and cubic B-splines by generalizing higher-order Voronoi diagrams. In Proc. ACM Sympos. Comput. Geom., pages 150-157, 2007.

[11] Online Encyclopedia of Integer Sequences. http://oeis.org/A216274.

[12] R. Pinchasi and G. Rote. On the maximum size of an anti-chain of linearly separable sets and convex pseudo-discs. Israel J. Math., 172:337-348, 2009.

[13] G. Rote. http://sarielhp.org/blog/?p=1951.

[14] N. Sauer. On the density of families of sets. Journal of Combinatorial Theory, Series A, 25:80-83, 1972.

[15] D. Schmitt and J.-C. Spehner. On Delaunay and Voronoi diagrams of order $k$ in the plane. In Proc. 3rd Canad. Conf. Comput. Geom., pages 29-32, 1991.

[16] S. Shelah. A combinatorial problem, stability and order for models and theories in infinite languages. Pacific J. Math., 41:247-261, 1972.

[17] J. Steiner. Einige Gesetze über die Theilung der Ebene und des Raumes. J. reine und angew. Math., 1:349-364, 1826.

[18] G. Tóth. Point sets with many k-sets. Discrete Comput. Geom., 26(2):187-194, 2001.

[19] V. N. Vapnik and A. Y. Chervonenkis. On the uniform convergence of relative frequences of events to their probabilities. Theory Probab. Appl., 16:264-280, 1971. 
[20] Wikipedia. http://en.wikipedia.org/wiki/Cake_number.

[21] Yu. A. Zak and V. M. Kotlov. The minimal quantity of separating hyperplanes in a classification problem (in russian). Kibernetika, 2:141-142, 1973.

[22] T. Zaslavsky. Facing up to Arrangements: Face-Count Formulas for Partitions of Space by Hyperplanes, volume 1 of Memoirs Amer. Math. Soc. 1975. 